Avaliação do custo e efetividade dos critérios de teste estruturais no contexto de programas concorrentes com memória compartilhada 



\title{
Avaliação do custo e efetividade dos critérios de teste estruturais no contexto de programas concorrentes com memória compartilhada
}

\author{
Silvana Morita Melo \\ Orientadora: Profa. Dra. Simone do Rocio Senger de Souza \\ Dissertação apresentada ao Instituto de Ciências \\ Matemáticas e de Computação - ICMC-USP, como \\ parte dos requisitos para obtenção do título de Mestre \\ em Ciências - Ciências de Computação e Matemática \\ Computacional. VERSÃO REVISADA
}


Ficha catalográfica elaborada pela Biblioteca Prof. Achille Bassi e Seção Técnica de Informática, ICMC/USP, com os dados fornecidos pelo(a) autor(a)

Morita Melo, Silvana
Avaliação do custo e efetividade dos critérios de
teste estruturais no contexto de programas
concorrentes com memória compartilhada / Silvana
Morita Melo; orientadora Simone do Rocio Senger de
Souza. -- São Carlos, 2012.
100 p.
Dissertação (Mestrado - Programa de Pós-Graduação en
Ciências de Computação e Matemática Computacional) --
Instituto de Ciências Matemáticas e de Computação,
Universidade de São Paulo, 2012.
1. Teste de software. 2. Programação concorrente.
S. Engenharia de software experimental. I. do Rocio
Senger de Souza, Simone, orient. II. Título.





\section{Agradecimentos}

Agradeço a Deus por ter me dado a vida e a capacidade de lutar pelas minhas ambições.

À minha famíla, pelo amor, apoio e confiança. Principalmente aos meus pais que sempre acreditaram e incentivaram meus sonhos.

Aos médicos Ricardo Inneco de Castro e Marcel Cammarosano, por sua dedicação, atenção e competência, as quais tornaram minha recuperação possível e consequentemente viabilizou a realização deste trabalho.

À todos amigos que conquistei durante o período do mestrado. Às minhas amigas: Maria, Joice, Arineiza, Valéria, Juliana, Vânia, Eliana e os amigos: Rodolfo, Harry, Draylson, Dionísio, Rafael, Lucas, Eduardo, Fabiano e aos demais colegas e professores do LabES, pelos momentos descontraídos e ótima companhia durante essa caminhada.

Ao amigo Vinícius Siqueira, pela paciência e auxílio com a análise estatística.

À FAPESP pelo apoio financeiro.

Em especial, aos meus pais adotivos Simone e Paulo, pela orientação, não apenas em relação ao mestrado, mas em todos os momentos que necessitei. 

O teste de programas concorrentes é uma atividade desafiadora, devido a fatores que não estão presentes em programas sequenciais, como comunicação, sincronização e não determinismo. Algumas técnicas de teste têm sido propostas para o contexto de programação concorrente, mas raramente sua aplicabilidade é avaliada por estudos teóricos ou experimentais. Este trabalho contribui nesse sentido, propondo e conduzindo um estudo experimental para avaliar o custo, eficácia e aspecto complementar dos critérios de teste estruturais para programas concorrentes no contexto de memória compartilhada, implementados usando o padrão PThreads (Posix Threads). A ferramenta de teste ValiPThread é usada para auxiliar a condução do experimento. Os programas usados no experimento foram selecionados de benchmarks, como o Inspect, Helgrind e Rungta. Esses benchmarks são comumente usados no estudo de técnicas de teste para programas concorrentes. Programas que resolvem problemas clássicos da programação concorrente também foram incluídos no estudo. Com base nos resultados obtidos foi definida uma estratégia de aplicação, considerando aspectos de custo e eficácia dos critérios de teste. Além disso, todo o material utilizado e gerado durante o experimento foi reunido em um pacote de laboratório, a fim de contribuir com a comunidade de pesquisa, possibilitando replicações e comparações desses critérios com outras técnicas de teste no contexto de programas concorrentes. 

Concurrent program testing is a challenging activity due to the communication, synchronization and nondeterminism of this application domain. Despite that, some testing techniques for concurrent programs have been proposed, but their applicability is rarely evaluated by theoretical or experimental studies. This work contributes in this direction proposing and conducting an experimental study to evaluate the cost, effectiveness and strength of structural testing criteria for multithreaded programs, implemented using the Pthreads standard (POSIX Threads). The testing tool ValiPThread is used to support the conduction of the experiment. The programs used in this experiment were selected from classical benchmarks, such as Inspect, Helgring and Rungta. These benchmarks are commonly used to study testing techniques for concurrent programs. We also include programs that solve concurrent classical problems. Based on the obtained results, we defined an application testing strategy, considering cost and effectiveness aspects of the testing criteria. Furthermore, all material used and generated during the experiment was incorporated in a lab package, in order to contribute with further research studies making possible replications and comparisons of these testing criteria with other testing techniques in context of concurrent programs. 

1 Introdução 1

1.1 Contexto e Motivação . . . . . . . . . . . . . . . . . . . . 1

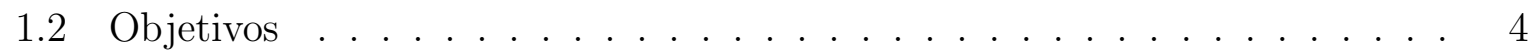

1.3 Organização do Trabalho . . . . . . . . . . . . . . . . . . 4

2 Fundamentação Teórica $\quad 7$

2.1 Considerações Iniciais . . . . . . . . . . . . . . . . . . . . . . . . . . . . . . . . . . . . .

2.2 Teste de Software . . . . . . . . . . . . . . . . . . . . . 8

2.2.1 Técnica Estrutural . . . . . . . . . . . . . . . . . . . . . 9

2.3 Programação Concorrente . . . . . . . . . . . . . . . . . . . 12

2.3.1 Pthreads - API POSIX para Threads . . . . . . . . . . . . . . . 15

2.4 Teste Estrutural de Programas Concorrentes . . . . . . . . . . . . . . . . 17

2.4.1 Experimentação em Teste de Programas Concorrentes . . . . . . . . 28

2.5 Considerações Finais . . . . . . . . . . . . . . . . . . . . . . 31

3 Definição, Planejamento e Operação do Experimento 33

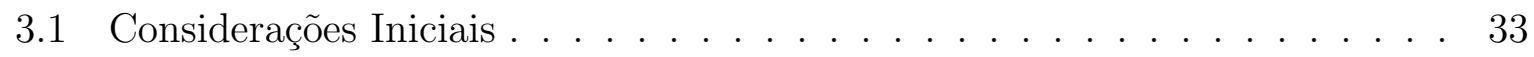

3.2 Definição do Experimento . . . . . . . . . . . . . . . . . . . . . . . . . . . . . . . . .

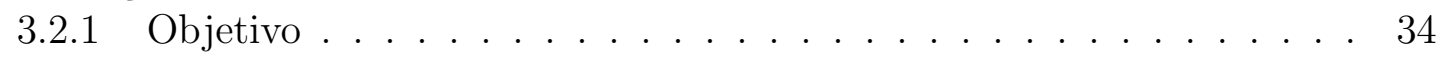

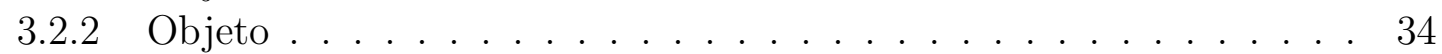

3.2 .3 Foco Qualitativo . . . . . . . . . . . . . . 34

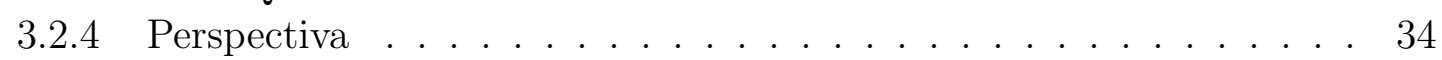

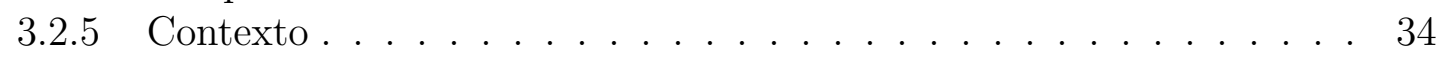

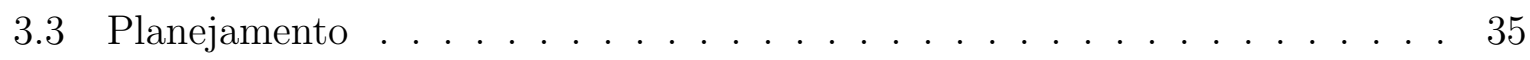

3.3 .1 Seleção do Contexto . . . . . . . . . . . . . . 35

3.3 .2 Definição das Hipóteses . . . . . . . . . . . . . . . . . . . . . . . . . . . . . . . . . . . 36

3.3 .3 Seleção dos Sujeitos . . . . . . . . . . . . . . . . . . . . . . . . . . . . . . 36

3.3 .4 Seleção das Variáveis . . . . . . . . . . . . . . . . . . . . . . . . 37

3.3.5 Projeto do Experimento . . . . . . . . . . . . . . . . . 37

3.3 .6 Descrição da Instrumentação . . . . . . . . . . . . . . . . . . . . . . 38

3.3.7 Ameaças a Validade do Experimento . . . . . . . . . . . . . . . . . 39

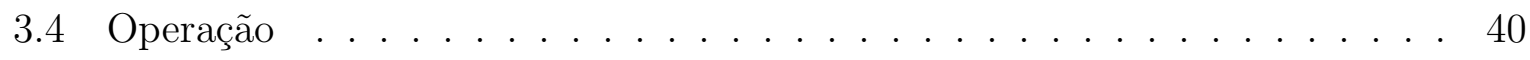

3.4 .1 Preparação . . . . . . . . . . . . . . . . . 41 
3.4 .2 Execução . . . . . . . . . . . . . . . . . . . . . . . . . . . . . . . . . . . . .

3.5 Considerações Finais . . . . . . . . . . . . . . . . . . . . . 44

4 Análise dos Resultados $\quad 45$

4.1 Considerações Iniciais . . . . . . . . . . . . . . . . . . . . . . 45

4.2 Estatística Descritiva . . . . . . . . . . . . . . . . . . 45

4.2 .1 Análise do Custo . . . . . . . . . . . . . . . . 46

4.2 .2 Análise da Eficácia . . . . . . . . . . . . . . . . . . . 51

4.2 .3 Análise do Strength . . . . . . . . . . . . . . . . . . . . . . . . . . . . . . . 55

4.3 Redução do Conjunto de Dados . . . . . . . . . . . . . . . . . . 61

4.4 Teste de Hipóteses . . . . . . . . . . . . . . . . . . . . . . . . . . . . . . . 62

4.4.1 Custo do Critério de Teste . . . . . . . . . . . . . . . . . 62

4.4 .2 Eficácia do Critério de Teste . . . . . . . . . . . . . 65

4.4 .3 Strength . . . . . . . . . . . . . . . . . . . . . . . . . . . . . . . . . . .

4.4.4 Síntese das Análises . . . . . . . . . . . . . . . . . . 72

4.5 Comparação entre Critérios Concorrentes e Sequenciais . . . . . . . . . . . 74

4.5.1 Definição das Hipóteses . . . . . . . . . . . . . . . . . . . . . . . . 74

4.5 .2 Teste de Hipóteses . . . . . . . . . . . . . . . . . . . 75

4.5.2.1 Análise da Eficácia . . . . . . . . . . . . . . . 75

4.5.2.2 Análise do Custo . . . . . . . . . . . . . . 76

4.5.3 Síntese das Análises . . . . . . . . . . . . . . . . . . . . . 78

4.6 Apresentação e Empacotamento . . . . . . . . . . . . . . . . . . . . . . . . 80

4.7 Considerações Finais . . . . . . . . . . . . . . . . . . . . . 81

5 Conclusão $\quad 83$

5.1 Caracterização da Contribuição . . . . . . . . . . . . . . . . . . . . . . . . . . . . . . . 83

5.2 Contribuições da Pesquisa . . . . . . . . . . . . . . . . . . . . 84

5.3 Trabalhos Futuros . . . . . . . . . . . . . . . . . . . . 84

$\begin{array}{ll}\text { Referências } & 92\end{array}$

$\begin{array}{ll}\text { A Resultados adicionais } & 93\end{array}$

A.1 Análise Descritiva para o Fator Strength . . . . . . . . . . . . . . . . 93 
2.1 Código fonte do programa hello.c implementado usando o padrão PThreads. 16

2.2 PCFGsm para o programa hello.c. . . . . . . . . . . . . . . 19

2.3 Arquitetura da ferramenta ValiPthread (Sarmanho, 2009). . . . . . . . . . 23

2.4 Conteúdo do arquivo All-edgesS.req. . . . . . . . . . . . . . . 25

2.5 Conteúdo do arquivo All-edgesS.aut . . . . . . . . . . . . . 26

2.6 Interpretação do autômato em modo texto. . . . . . . . . . . . . . 26

2.7 Autômato finito em modo gráfico. . . . . . . . . . . . . . . . 27

2.8 Saída do programa hello.c para o caso de teste $1 . \quad \ldots$. . . . . . . . . 27

2.9 Traces de execução do programa hello.c para o caso de teste 1. . . . . . . . 27

2.10 Resultado da cobertura do critério all-s-edges. . . . . . . . . . . . . 28

4.1 Custo relacionado ao tamanho do conjunto de casos de teste adequado. . . 48

4.2 Custo dos critérios considerando o conjunto adequado. . . . . . . . . . . 48

4.3 Custo relacionado à quantidade de elementos requeridos. . . . . . . . . . . 51

4.4 Custo dos critérios considerando o número de elementos requeridos. . . . . 51

4.5 Custo relacionado ao número de elementos não executáveis. . . . . . . . . . 52

4.6 Custo dos critérios considerando o número de elementos não executáveis. . 52

4.7 Eficácia dos critérios de teste. . . . . . . . . . . . . . . . . . . . 54

4.8 Eficácia dos critérios de teste. . . . . . . . . . . . . . . . . . . 54

4.9 Dificuldade de satisfação para o critério all-nodes quando aplicado aos demais critérios. . . . . . . . . . . . . . . . . 56

4.10 Dificuldade de satisfação para o critério all-p-nodes quando aplicado aos demais critérios. . . . . . . . . . . . . . . . . 57

4.11 Dificuldade de satisfação para o critério all-w-nodes quando aplicado aos demais critérios . . . . . . . . . . . . . . . . . . . . 59

4.12 Dificuldade de satisfação para o critério all-edges quando aplicado aos demais critérios. . . . . . . . . . . . . . . . . 60

4.13 Dificuldade de satisfação para o critério all-s-edges quando aplicado aos demais critérios. . . . . . . . . . . . . . . . . . 61

4.14 Resultados do teste de Tukey para o custo em relação à quantidade de elementos requeridos. . . . . . . . . . . . . . . . 65

4.15 Resultados do teste de Tukey para o custo em relação à quantidade de elementos não-executáveis. . . . . . . . . . . . . . . . 66 
4.16 Strength para o critério all-nodes $(\mathrm{AN}) \ldots \ldots \ldots \ldots$. . . . . . . 67

4.17 Strength para o critério all-w-nodes (ANW) . . . . . . . . . . . 68

4.18 Strength para o critério all-edges (AE) . . . . . . . . . . . . . . 69

4.19 Strength para o critério all-p-nodes (ANP) . . . . . . . . . . . . . 69

4.20 Strength para o critério all-c-uses (ACU) . . . . . . . . . . . . 70

4.21 Strength para o critério all-p-uses (APU) . . . . . . . . . . . . 70

4.22 Strength para o critério all-comm-p-uses (ACPU) . . . . . . . . . . . 71

4.23 Strength para o critério all-comm-c-uses (ACCU) . . . . . . . . . . . 71

4.24 Strength para o critério all-sync-uses (ASU) . . . . . . . . . . . . . . 72

4.25 Strength para o critério all-s-edges (AES) . . . . . . . . . . . 73

A.1 Dificuldade de satisfação para o critério all-c-uses quando aplicado aos demais critérios. . . . . . . . . . . . . . . . . . . . 94

A.2 Dificuldade de satisfação para o critério all-comm-c-uses quando aplicado aos demais critérios. . . . . . . . . . . . . . . . . . . . . 94

A.5 Dificuldade de satisfação para o critério all-comm-p-uses quando aplicado aos demais critérios. . . . . . . . . . . . . . . . . 96

A.3 Dificuldade de satisfação para o critério all-p-uses quando aplicado aos demais critérios. . . . . . . . . . . . . . . . . . . . 97

A.4 Dificuldade de satisfação para o critério all-sync-uses quando aplicado aos demais critérios. . . . . . . . . . . . . . . . . . 97 


\section{Lista de Tabelas}

3.1 Programas selecionados. . . . . . . . . . . . . . . . . . . . 42

3.2 Classificação dos erros inseridos. . . . . . . . . . . . . . . . . . . 44

4.1 Custo por critério considerando o conjunto de casos de teste adequado . . . 47

4.2 Custo: Elementos requeridos/Elementos não executáveis. . . . . . . . . . . 50

4.3 Eficácia por critério de teste. . . . . . . . . . . . . . . . 53

4.4 Eficácia por tipo de defeito. . . . . . . . . . . . . . . . . 53

4.5 Aplicação do conjunto de casos de teste adequado ao critério all-nodes (AN) aos demais critérios. . . . . . . . . . . . . . 56

4.6 Aplicação do conjunto de casos de teste adequado ao critério all-p-nodes (ANP) aos demais critérios. . . . . . . . . . . . . . 57

4.7 Aplicação do conjunto de casos de teste adequado ao critério all-w-nodes (ANW) aos demais critérios. . . . . . . . . . . . . . . . . 58

4.8 Aplicação do conjunto de casos de teste adequado ao critério all-edges (AE) aos demais critérios. . . . . . . . . . . . . . . . . . . . 59

4.9 Aplicação do conjunto de casos de teste adequado ao critério all-s-edges (AES) aos demais critérios. . . . . . . . . . . . . . . . 60

4.10 Resultados do teste Kruskal-Wallis para o custo em relação ao conjunto de casos de teste adequado. . . . . . . . . . . . . . . . . 63

4.11 Resultados do teste ANOVA para o custo considerando a quantidade de elementos requeridos. . . . . . . . . . . . . . . . . . 64

4.12 Resultados do teste ANOVA para o custo considerando a quantidade de elementos não-executáveis. . . . . . . . . . . . . . . . . . 65

4.13 Resultados do teste ANOVA para a eficácia do critério de teste. . . . . . . 67

4.14 Resultados do teste de Friedman com análise par a par. . . . . . . . . . . 76

4.15 Resultado do teste de Friedman. . . . . . . . . . . . . . . . 76

4.16 Resultados do teste de Wilcoxon pareado para o custo considerando o conjunto adequado. . . . . . . . . . . . . . . . . . . 77

4.17 Resultados do teste de Wilcoxon pareado para o custo considerando o número de elementos requeridos. . . . . . . . . . . . . . . . . 78

4.18 Resultados do teste de Wilcoxon pareado para o custo considerando a quantidade de elementos não executáveis. . . . . . . . . . . . . . . . 79

4.19 Resultados do teste de hipóteses. . . . . . . . . . . . . . . . . . . . . 79 
A.1 Aplicação do conjunto de casos de teste adequado ao critério all-c-uses (ACU) aos demais critérios.. . . . . . . . . . . . . . . . . . 95

A.2 Aplicação do conjunto de casos de teste adequado ao critério all-comm-c-uses (ACCU) aos demais critérios. . . . . . . . . . . . . . . 96

A.3 Aplicação do conjunto de casos de teste adequado ao critério all-p-uses (APU) aos demais critérios. . . . . . . . . . . . . . . . . . . 98

A.4 Aplicação do conjunto de casos de teste adequado ao critério all-sync-uses (ASU) aos demais critérios. . . . . . . . . . . . . . . . . . . 99

A.5 Aplicação do conjunto de casos de teste adequado ao critério all-comm-p-uses (ACPU) aos demais critérios. . . . . . . . . . . . . . . 100 


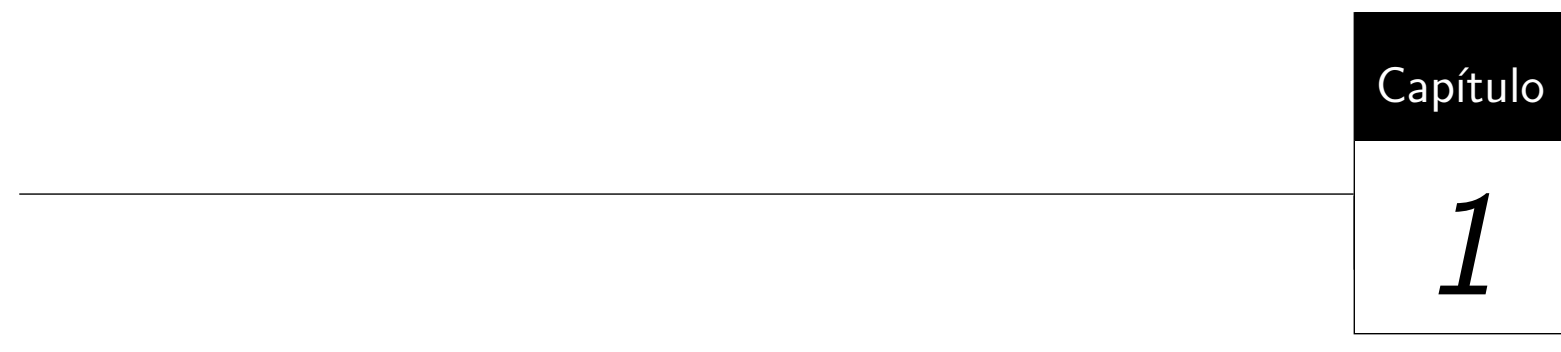

Introdução

\subsection{Contexto e Motivação}

A atividade de teste de software é uma das mais onerosas do processo de desenvolvimento de software. Segundo Pressman (2001) ela pode consumir grande parte do custo total do projeto, porém não pode ser desprezada quando se almeja construir um produto de qualidade e livre de defeitos. Sabe-se que para comprovar que um produto está livre de defeitos é necessário sua execução utilizando todo o domínio de entrada, uma atividade impraticável quando é observado o tamanho do domínio de entrada que, até mesmo para programas triviais, pode ser imenso (Delamaro et al., 2007). Uma alternativa é criar subconjuntos do domínio de entrada formados por uma quantia reduzida de elementos, mas que representem o domínio como um todo. Para seleção desses elementos são utilizados técnicas e critérios de teste.

Critérios de teste são definidos para apoiar a seleção de dados de teste, sistematizando a escolha desses dados de teste, guiados por informações oriundas do programa em teste. Essas informações dão origem a diferentes técnicas de teste: funcional, que usa informações sobre a especificação do programa, estrutural, que usa informações sobre a estrutura interna do programa e a técnica baseada em erros, que usa informações sobre os erros mais comuns cometidos no processo de desenvolvimento (DeMillo et al., 1978). Cada técnica apresenta um conjunto de critérios de teste, os quais apresentam regras que estabelecem quais informações devem ser exercitadas pelos dados de teste selecionados. 
No contexto de programas sequenciais inúmeras pesquisas têm sido desenvolvidas com o intuito de definir técnicas e critérios para validação e teste de programas, dentre elas pode-se citar: (Beizer, 1990; Delamaro et al., 2007; Maldonado, 1991; Myers e Sandler, 2004; Pressman, 2001; Weyuker et al., 1991). Esses trabalhos oferecem fundamentos básicos e norteiam a criação de novos critérios de teste.

Com os avanços computacionais principalmente na busca por maior desempenho computacional, surgem máquinas paralelas e inerentemente a necessidade por software paralelo. Computadores paralelos dispõem de múltiplos elementos de processamento, que trabalham simultaneamente para resolver um mesmo problema em menor tempo. Aplicações desenvolvidas para essas arquiteturas são por natureza mais complexas que programas sequenciais, pois envolvem a execução simultânea de processos que interagem para realizar as tarefas. A implementação desses processos depende do uso de primitivas para definir inicialização e finalização de processos paralelos, além de comunicação e sincronização de processos. Dentre essas primitivas, as que merecem maior atenção são as que tratam a comunicação e sincronização entre os processos pois refletem no resultado final da aplicação.

Existem dois paradigmas fundamentais para coordenar a interação entre os processos, classificados de acordo com a memória disponível (Almasi e Gottlieb, 1989; Souza et al., 2007b). O paradigma de memória distribuída estabelece a comunicação e a sincronização entre os processos por meio da passagem de mensagens, onde cada processo é executado por uma unidade de processamento que possui seu próprio espaço de endereçamento. No paradigma de memória compartilhada a sincronização é obtida com a utilização de semáforos e monitores e variáveis compartilhadas são usadas para permitir a comunicação entre os processos que compartilham o mesmo espaço de endereçamento. Esses dois paradigmas apresentam características diferentes, as quais necessitam ser consideradas durante os testes.

O teste de aplicações concorrentes é mais complicado, pois além das dificuldades inerentes à atividade de teste, novos desafios são impostos devido principalmente ao comportamento não determinístico onde diferentes sequências de sincronização podem ser obtidas com utilização de uma mesma entrada. Nesse sentido, Yang e Pollock (2003) reúnem os principais desafios impostos ao teste de programas concorrentes, que são: 1) Necessidade de desenvolver técnicas de análise estática apropriadas para programas concorrentes; 2) Detectar situações não desejadas como: erros de sincronização, comunicação, de fluxo de dados e de deadlock; 3) Reproduzir uma execução com a mesma entrada de teste e forçar a execução de um caminho na presença de não determinismo; 4) Gerar uma representação do programa concorrente que capture informações pertinentes ao teste; 5) Investigar a aplicação de critérios de teste sequenciais para programas concorrentes; 6) De- 
senvolver um framework que implemente a aplicação de critérios de teste estrutural para programas paralelos; 7) Projetar critérios de fluxo de dados para programas concorrentes, considerando troca de mensagens e variáveis compartilhadas (Souza et al., 2008b).

Baseados nesses desafios e, em especial, em desenvolver técnicas de validação para programas concorrentes, vários trabalhos têm sido desenvolvidos e comprovam a aplicabilidade do uso da técnica estrutural para o teste de programas concorrentes (Edelstein et al., 2003; Koppol et al., 2002; Saini, 2005; Souza et al., 2008b; Taylor et al., 1992; Wong et al., 2005; Yang et al., 1998).

O grupo de pesquisa em Engenharia de Software do ICMC/USP tem desenvolvido trabalhos relacionados à definição de mecanismos de apoio à atividade de teste de programas concorrentes. Modelos de teste específicos para programação concorrente que utilizam o paradigma de passagem de mensagem foram propostos pelo grupo, e com o objetivo de validar essas propostas, a ferramenta Valipar (Souza et al., 2005) foi desenvolvida e instanciada para dois ambientes de passagem de mensagem: o PVM (Parallel Virtual Machine) e o MPI (Message Passing Interface) (Hausen e Vergilio, 2005; Souza et al., 2008a). A definição de critérios de teste contribui com o processo de teste de aplicações paralelas, fornecendo uma medida de cobertura sobre o processo de teste, a qual pode ser utilizada para avaliar a qualidade da atividade de teste.

Tendo como base os trabalhos anteriores, Sarmanho (2009) apresenta um modelo de teste para ser aplicado à programas concorrentes que utilizam memória compartilhada (PThreads). Para isso um grafo denominado PCFGsm (Parallel Control Flow Graph for Shared Memory) foi definido, o qual considera informações sobre o fluxo de controle e de dados de cada thread, informações sobre as possíveis sincronizações (considerando as primitivas post e wait) e informações sobre a comunicação entre as threads, dada pelo uso de variáveis compartilhadas. As ideias propostas foram implementadas em uma ferramenta de apoio, denominada ValiPThread, que segue a mesma arquitetura definida pela Valipar (Souza et al., 2005), adaptando os módulos necessários (Sarmanho, 2009). Nesse trabalho um conjunto formado por doze critérios estruturais foram definidos, contemplando os aspectos extraídos pelo modelo de teste. Esses critérios foram definidos tentando evidenciar defeitos típicos nesse contexto, relacionados ao fluxo de controle, fluxo de dados, sincronização e comunicação entre threads. Entretanto, a efetividade dos critérios não foi analisada por meio de estudos teóricos ou experimentais.

Este projeto de mestrado visa a contribuir nesse cenário, estabelecendo e aplicando um estudo experimental para avaliar a efetividade dos critérios de teste definidos para programas concorrentes no contexto de memória compartilhada. Este estudo é desenvolvido em sintonia com os pontos básicos de comparação teórica e empírica de critérios de teste: custo, eficácia e strength (Mathur e Wong, 1993; Souza, 1996; Souza et al., 2007a; Wong 
et al., 1995), fornecendo evidências dessas métricas para o contexto de aplicações concorrentes. Nesse contexto, custo está relacionado ao trabalho necessário para satisfazer um determinado critério de teste, eficácia corresponde à habilidade que um critério possui de revelar mais defeitos que outro e strength está relacionado à probabilidade de satisfazer um critério tendo sido satisfeito outro (Wong et al., 1995). Para o desenvolvimento deste trabalho, um benchmark de programas foi selecionado, de modo a contribuir com o estabelecimento de um Pacote de Laboratório, que poderá ser posteriormente usado como material de apoio ao ensino e treinamento. Um Pacote de Laboratório é composto de todos os artefatos utilizados e gerados durante um experimento de modo que o mesmo possa ser replicado e validado em outros domínios ou contextos. Esse conceito tem sido utilizado no contexto de Engenharia de Software Experimental (Wohlin et al., 2000) e será aplicado neste trabalho. Por fim, uma estratégia incremental de aplicação desses critérios será definida com objetivo de auxiliar a realização da atividade de teste com baixo custo e alta eficácia em revelar defeitos.

\subsection{Objetivos}

Este trabalho tem como objetivo a caracterização e aplicação de um estudo experimental para avaliar a eficácia em detectar defeitos, o custo de aplicação e o aspecto complementar dos critérios de teste estruturais definidos para programas concorrentes com memória compartilhada, implementados utilizando o padrão PThreads (POSIX Threads). Esse estudo permitirá obter evidências a respeito dos critérios estudados e gerar material que permita o desenvolvimento de investigações futuras.

\subsection{Organização do Trabalho}

No Capítulo 2 são apresentados os conceitos fundamentais do teste de software, com ênfase na técnica estrutural de teste. Introduz-se a programação concorrente, os principais conceitos e terminologias utilizados, as principais linguagens e paradigmas de suporte à programação concorrente, destacando o paradigma de memória compartilhada, e a API PThreads. É abordado o teste de programas concorrentes dando uma visão geral das principais iniciativas nessa área e alguns trabalhos que, assim como este, propõem estudos experimentais nesse contexto.

A partir do Capítulo 3 são descritas as principais fases do processo experimental, sendo que neste capítulo são apresentadas a definição, o planejamento e a operação do experimento realizado. No Capítulo 4 os resultados obtidos com a execução do experimento, são analisados a fim de obter conclusões relevantes à pesquisa. 


\section{CAPÍTULO 1. INTRODUÇÃO}

Por fim, no Capítulo 5 são apresentadas as principais conclusões obtidas por meio da realização do estudo experimental, as principais contribuições alcançadas e algumas propostas interessantes a trabalhos futuros. 



$=$

\section{Fundamentação Teórica}

\subsection{Considerações Iniciais}

Este capítulo aborda as principais áreas envolvidas nesta pesquisa, o teste de software, a técnica estrutural de teste de software, a programação concorrente, o teste estrutural de programas concorrentes e o uso de experimentação no teste de programas concorrentes.

O capítulo está organizado da seguinte forma: na Seção 2.2 é definida a atividade de teste, são apresentadas as técnicas usadas durante essa atividade, assim como sua importância na garantia da qualidade dos produtos de software e do processo de desenvolvimento.

Na Seção 2.3 são introduzidos os conceitos e terminologias básicas usadas em programas concorrentes. São abordados paradigmas de passagem de mensagem e memória compartilhada, porém com ênfase no paradigma que utiliza memória compartilhada que faz parte do escopo deste trabalho. É descrita também, a API POSIX Threads (PThreads), uma API padrão especificada pelo IEEE para a construção de programas concorrentes usando threads.

Na Seção 2.4 são apresentadas inciativas voltadas ao teste estrutural de programas concorrentes. Na Seção 2.4.1 são descritos alguns trabalhos encontrados na literatura que, assim como este projeto, propõe a utilização da experimentação para validar suas propostas. Por fim, a Seção 2.5 traz algumas considerações sobre os assuntos abordados neste capítulo. 


\subsection{Teste de Software}

Com objetivo de identificar e eliminar defeitos presentes no produto de software atividades de VV\&T (Verificação, Validação e Teste) visam garantir a qualidade do software produzido. Dentre essas, o teste de software é uma atividade que visa assegurar que o software faça somente aquilo que está descrito na sua especificação. Os objetivos principais do processo de teste são (Myers e Sandler, 2004):

- exercitar um programa a procura de defeitos;

- desenvolver casos de teste com grandes chances de encontrar defeitos ainda não descobertos;

- realizar um teste de qualidade, teste de qualidade é aquele que revela defeitos ainda não descobertos.

Defeitos são identificados em todos os níveis do processo de desenvolvimento do software. Portanto, a atividade de teste deve ser realizada durante todo o ciclo de desenvolvimento do software. Assim, o processo de teste é conduzido em três fases distintas (Binder, 1999; Delamaro et al., 2007; Maldonado, 1991; Pressman, 2001):

- teste de unidade, nessa fase cada módulo do sistema é testado separadamente à procura de defeitos de lógica e de implementação;

- teste de integração, visa identificar defeitos de comunicação entre os módulos testados na fase anterior. Durante a integração entre as partes do sistema, são testadas as várias interfaces do sistema, ou seja, a estrutura formada pelos módulos;

- teste de sistema é realizado ao final da integração de todos os módulos, verificando se os elementos foram devidamente integrados e realizam as funções a eles atribuídas, além de verificar a existência de defeitos e resultados não condizentes com a especificação, colocando a prova o sistema como um todo.

Em cada uma dessas fases, o processo de teste é sempre realizado em quatro etapas distintas, planejamento de testes, projeto de casos de teste, execução e avaliação dos resultados do teste (Beizer, 1990; Maldonado, 1991; Myers e Sandler, 2004; Pressman, 2001).

Tendo em vista o alto custo da atividade de teste em comparação às demais atividades que constituem o processo de desenvolvimento de software, é de suma importância que o teste seja realizado de maneira rápida e demandando pouco esforço. Assim, dentre as etapas citadas acima, a que merece maior atenção é a de projeto de casos de teste que 
consiste na seleção dos casos de teste que tenham maior chance de identificar defeitos, poupando esforço desnecessário com a execução de casos de teste ineficazes. Para auxiliar essa atividade são desenvolvidas técnicas e critérios de teste (Delamaro et al., 2007).

Técnicas de teste provêm normas para o projeto sistemático de casos de teste que exercitem tanto a lógica interna dos componentes do software, como seus domínios de entrada e saída (Pressman, 2001).

Segundo Zhu (1996) a qualidade dos testes está associada ao critério de teste utilizado, pois é ele quem define quais as propriedades ou requisitos deverão ser usados na avaliação dos testes. Os critérios de teste podem ser definidos de acordo com a função que desempenham, critérios de seleção de dados de teste são procedimentos utilizados para escolha de casos de teste, e critérios de adequação dos dados de testes são usados para avaliar dados de testes. Diz-se que dado um programa $P$ a ser testado e um conjunto de caso de testes $T$, $T$ é $C$-adequado para a realização do teste de $P$ se $T$ satisfaz os requisitos de teste estabelecidos pelo critério $C$ (Frankl, 1987; Zhu, 1996).

As técnicas e os critérios de teste são fundamentais na seleção de casos de teste, pois por meio deles consegue-se minimizar o número de casos de teste, garantindo que partes específicas do código sejam cobertas pelos testes. As técnicas de teste diferem entre si pela origem da informação utilizada para seleção de dados de teste. A técnica funcional baseia-se na especificação do produto de software, a técnica estrutural utiliza a estrutura interna do programa e a técnica baseada em defeitos utiliza as falhas típicas cometidas pelos programadores durante o desenvolvimento do software. Dada a importância para este trabalho, a técnica estrutural é descrita em mais detalhes a seguir.

\subsubsection{Técnica Estrutural}

O teste estrutural ou teste de caixa branca utiliza o conhecimento da estrutura interna do programa para derivação dos casos de teste. Os critérios pertencentes à técnica estrutural são classificados com base na complexidade, no fluxo de controle e no fluxo de dados (Frankl, 1987; Pressman, 2001; Sugeta, 1999; Weyuker et al., 1991).

Critérios baseados na complexidade do software utilizam uma representação do fluxo de controle do programa para calcular a complexidade lógica do programa e, a partir dela, derivar requisitos de teste. A medida da complexidade lógica de um programa é denominada complexidade ciclomática. A complexidade ciclomática define o número de caminhos linearmente independentes, ou seja, caminhos que introduzam novos conjuntos de instruções de processamento ou novas condições. Um caminho corresponde à uma sequência de nós a serem exercitados em um programa (Maldonado, 1991). Esses caminhos definem o conjunto básico de casos de teste de um programa e estabelece um limite máximo para o número de testes que deve ser realizado para garantir que todas as instruções sejam 
executadas pelo menos uma vez. Um critério baseado em complexidade bastante utilizado é o critério de McCabe (McCabe, 1976), que utiliza a medida da complexidade ciclomática para derivação dos casos de teste (Pressman, 2001).

Critérios baseados no fluxo de controle derivam seus requisitos de teste com base nas informações de controle existentes no programa, ou seja, informações relacionadas a desvios ou repetições. Para isso, uma representação gráfica do programa conhecida como Grafo de Fluxo de Controle (GFC) ou Grafo de Programa é utilizada. No GFC, um programa $P$ é representado por um conjunto de nós que correspondem a blocos indivisíveis de comando e por arestas que representam o controle do fluxo de informações entre os nós. A partir desse grafo, caminhos de execução, ou seja, uma sequência nós do programa são estabelecidos, os quais iniciam em um nó de entrada e terminam em um nó de saída do grafo.

Essa representação serve de auxílio para a realização do teste, usado como base na aplicação e avaliação dos critérios e para derivar casos de teste. Os testes são realizados com base na implementação, por meio do controle da execução do programa. Dentre os critérios baseados no fluxo de controle do programa, os mais utilizados são:

- Todos-Nós: requer que cada nó, ou seja, cada comando do programa seja executado pelo menos uma vez durante a execução do programa em teste;

- Todos-Arcos: requer que todos os desvios entre os blocos ou arcos do GFC sejam percorridos ao menos uma vez;

- Todos-Caminhos: requer que todos os caminhos sejam percorridos ao menos uma vez. Esse critério é subdividido de acordo com o tipo de caminho a ser coberto;

- Todos-Caminhos-Completos: requer a cobertura de todos os caminhos completos. Um caminho completo é aquele em que o primeiro nó é o nó de entrada e o último nó é o nó de saída.

- Todos-Caminhos-Simples: exige que sejam percorridos todos os caminhos simples. Caminho simples é aquele onde todos os nós que o constituem são distintos, exceto possivelmente o primeiro e o último;

- Todos-Caminhos-Livres-de-Laços: requer que todos os caminhos livres de laço sejam percorridos ao menos uma vez pelo conjunto de sequências de teste. Caminho livre de laço é um caminho simples onde todos os nós são distintos inclusive o primeiro e o último.

Os critérios baseados no fluxo de dados utilizam informações sobre as variáveis definidas e usadas pelo programa para os testes. Nesse caso, o GFC é estendido com informações 
sobre o fluxo de dados, sendo denominado Grafo Def-Uso (Rapps e Weyuker, 1985). O Grafo Def-Uso é similar ao GFC, porém contém informações adicionais sobre associações entre pontos do programa onde ocorre definição de variáveis, ou seja, onde são atribuídos valores a uma variável, e pontos nos quais esse valor é utilizado, ou seja, onde é feito um uso ou referência à variável. Os requisitos de teste são determinados com base nessas associações.

O uso de variáveis pode ser classificado em dois tipos distintos: c-uso quando a utilização da variável afeta diretamente uma computação sendo realizada ou permite que o resultado de uma definição anterior possa ser observado, nesse tipo de uso o valor da variável é modificado, e p-uso quando o uso da variável afeta diretamente o fluxo de controle do programa não alterando seu valor. Geralmente no grafo c-usos são associados aos nós e p-usos às arestas (Maldonado, 1991).

Um conceito importante utilizado por esses critérios é o de caminho livre de definição. Um caminho é dito livre de definição quando uma variável $x$ é definida em um nó $i$, utilizada em um nó $j$ ou em um arco que chega a $j$, e o caminho $\left(i, n_{1}, \ldots, n_{m}, j\right), m>0$ não apresente nenhuma definição de $x$ nos nós $\left(n_{1}, \ldots, n_{m}\right)$ percorrido do nó $i$ ao nó $j$ e do nó $i$ ao arco $\left(n_{m}, j\right)$ (Delamaro et al., 2007). Outro conceito relevante é o Du-caminho, um caminho simples (sem repetição de nós exceto possivelmente o primeiro e o último) livre de definição em relação a uma variável $x$ do nó $i$ até o nó $j$ que possui uma definição da variável $x$ no nó $i$ e um uso no nó $j$ (Maldonado, 1991).

Os grafos de fluxo de controle e Def-Uso são a base para extração dos elementos requeridos para a cobertura dos critérios de teste propostos. Rapps e Weyuker (1985) propuseram um conjunto de critérios baseados em fluxo de dados, onde os mais conhecidos são:

- Todas-Definições: requer que cada definição de variável seja exercitada pelo ao menos uma vez por um p-uso ou c-uso;

- Todos-Usos: requer que todas as associações de uma variável sejam exercitadas pelos casos de teste, por meio de pelo menos um caminho livre de definição. Uma associação pode ser definida como o par formado pela definição de uma variável e seu subsequente uso. O critério Todos-Usos pode ser especializado em Todos-p-Usos, Todos-p-Usos/Alguns-c-Usos e Todos-c-Usos/Alguns-p-Usos;

- Todos-Du-Caminhos: requer que toda associação entre uma definição de variável e seus subsequentes $p$-usos ou c-usos sejam executados por todos os caminhos livres de definição e livres de laço.

Os critérios propostos por Rapps e Weyuker (1985) exigem a ocorrência explícita do uso de variáveis definidas. Como uma alternativa a esses critérios, Maldonado (1991) 
propôs um novo grupo de critérios, onde o uso da variável não precisa estar explicita, basta que seja possível um caminho livre de definição em relação a variável definida. Esses critérios são denominados critérios Potenciais-Usos e subdividem-se em:

- Todos-Potenciais-Usos: requer que pelo menos um caminho livre de definição de uma variável $x$ definida em um nó $i$ para todo nó e toda aresta alcançável a partir de $i$ seja exercitada;

- Todos-Potenciais-Usos/Du: requer que pelo menos um Potencial-Du-Caminho com relação à variável $x$ definida em $i$ para todo nó e para aresta alcançável a partir de $i$ seja exercitada;

- Todos-Potenciais-Du-Caminhos: requer que todos os Potenciais-Du-Caminhos com relação a todas as variáveis $x$ definidas e todos os nós e arestas alcançáveis a partir dessa definição sejam exercitados.

Critérios pertencentes a diferentes técnicas de teste são complementares e devem ser usados em conjunto, pois cobrem classes distintas de defeitos. O estabelecimento de estratégias incrementais que explorem as diversas características dos critérios favorece a obtenção de casos de teste mais abrangentes, confiáveis e de qualidade.

Visando estabelecer estratégias de teste mais eficazes, estudos experimentais têm sido conduzidos pela academia a fim de avaliar aspectos como custo, strength e eficácia dos critérios desenvolvidos. Proporcionado uma base sólida para transformação de estudos teóricos em prática, favorecendo o desenvolvimento de ferramentas (Delamaro et al., 2007).

No contexto de programas tradicionais (ou programas com características sequenciais), autores têm proposto várias técnicas e critérios para a sua validação, considerando questões importantes em relação à atividade de teste como seleção de casos de teste e avaliação da adequação dos casos de teste (Beizer, 1990; Coward, 1988; DeMillo et al., 1978; Herman, 1976; Howden, 1976; Laski e Korel, 1983; Maldonado, 1991; Myers e Sandler, 2004; Ural e Yang, 1988). Esses trabalhos oferecem a base fundamental para que novos modelos de teste sejam propostos considerando novos paradigmas e metodologias de desenvolvimento de software e possam ser aplicados a outros domínios e outros paradigmas de programação, como desenvolvimento orientado a objetos, a aspectos, entre outros.

\subsection{Programação Concorrente}

As vantagens no uso da programação concorrente em contrapartida à programação sequencial está principalmente ligada ao fato de que a programação concorrente possibilita ocupar de maneira mais inteligente os recursos que a aplicação dispõe evitando a ociosidade de recursos presente no processamento sequencial de dados, aumentando a eficácia 
e diminuindo os custos (Grama et al., 2003; Tanenbaum, 1995). A computação paralela apresenta-se como uma alternativa à necessidade por maior desempenho computacional, diminuindo o tempo gasto com problemas complexos. Ela foi empregada inicialmente na solução de problemas que exigiam grande quantidade de processamento, principalmente em áreas como: previsão climática, dinâmica molecular, projetos estruturais, cosmologia, biologia e dinâmica dos fluídos (Quinn, 2003). Atualmente a computação paralela pode ser encontrada em outras áreas de atuação, como grandes sistemas de bancos de dados, servidores web e servidores de web services (Grama et al., 2003). Os princípios nestas áreas de atuação permanecem os mesmos: divisão do problema a ser resolvido e execução paralela de diferentes módulos, a fim de se obter redução no tempo de execução, maior tolerância a falhas e soluções mais elegantes para problemas intrinsecamente paralelos (Grama et al., 2003).

Na computação distribuída, a execução de programas pode ser realizada através da criação de processos ou threads. Um processo nada mais é que o conjunto formado pelo software em execução e as informações necessárias para a sua execução, como os valores do contador de programa ( $P C$ - program counter), dos registradores e das variáveis. Cada processo possui um espaço próprio de endereçamento que conterá o código e os dados do programa além de outras informações utilizadas na execução e controle de sua execução. Diferentemente dos processos, as threads (ou lightweight processes - processos leves) compartilham um mesmo espaço de endereçamento, que é o espaço de endereçamento do processo pai, porém com seu próprio $P C$, pilha e registradores. Elas possuem as mesmas variáveis globais ou seja compartilham variáveis, o que permite a cooperação entre as threads na realização de uma tarefa. Para um aumento de desempenho, o ideal seria a execução de múltiplas threads (multithreads) de maneira paralela (multiprocessamento) ou de maneira concorrente (monoprocessamento). Threads podem ser consideradas uma maneira do processo paralelizar partes do código que serão escalonadas para execução sobre a CPU (Tanenbaum, 1995).

Para o desenvolvimento de processos concorrentes é necessário o uso de primitivas fundamentais que não estão presentes no desenvolvimento sequencial, tais como: definir quais processos serão executados concorrentemente; como inicializar e finalizar processos concorrentes; e como coordenar e determinar a interação entre os processos concorrentes durante a execução (Almasi e Gottlieb, 1989).

A interação entre processos é realizada por meio de variáveis compartilhadas ou troca de mensagens. A troca de mensagens ocorre quando há interação entre processos que não compartilham o mesmo espaço de endereçamento. Neste caso, tanto a comunicação quanto a sincronização são feitas através das primitivas básicas send e recv, usadas respectivamente para enviar e receber dados entre pares de processos. Quando estas primitivas 
são síncronas, elas também permitem a sincronização entre os processos. Há diferentes modos de operação das primitivas send e recv, sendo que elas podem ser classificadas como bloqueantes e não bloqueantes, síncronas e assíncronas, com buffer e sem buffer, ponto-a-ponto ou coletivas (Grama et al., 2003).

Quando a interação ocorre entre threads, que compartilham o mesmo espaço de endereçamento, há comunicação através do uso de variáveis compartilhadas e a sincronização é feita com o auxílio de diferentes primitivas como, por exemplo semáforos e monitores (Tanenbaum, 1995). A sincronização por meio de variáveis compartilhadas pode ser classificada em: exclusão mútua e sincronização condicional (Andrews, 1991).

A sincronização condicional garante o bloqueio de uma thread até que uma condição se torne verdadeira e ela possa prosseguir sua execução. O objetivo é coordenar a computação das threads.

A exclusão mútua garante que regiões críticas do programa sejam acessadas de maneira síncrona, ou seja, uma thread por vez. Existem vários mecanismos de sincronização que garantem exclusão mútua e a sincronização condicional entre processos. Dentre eles podemos citar:

- Semáforos (Dijkstra, 1965): um semáforo pode ser definido como uma variável inteira não negativa para contar o número de sinais já emitidos e que permitem a continuidade da execução de uma thread. Se o recurso compartilhado está disponível o valor do semáforo é maior que zero, caso contrário seu valor é zero. Duas operações atômicas são implementadas como system calls (chamadas de sistema), são elas o up e down. Quando o processo executa um down em um semáforo com valor maior que zero ele decrementa o valor do semáforo e continua a execução, se o valor for igual a zero ele é colocado em estado de espera (sleeping) e é automaticamente colocado na fila de pronto para execução quando o outro processo liberar o recurso usando o up. A atomicidade das instruções garantem a sincronização e evita condições de disputa.

- Mutexes: uma versão mais simplificada dos semáforos, cujo contador possui o valor 1 ou 0 . Assim, o mutex é uma variável utilizada para gerenciar exclusão mútua que pode conter dois estados: bloqueado (lock) e desbloqueado (unlock). Quando um processo ou thread tenta acessar a região crítica ele usa o procedimento mutex_lock, se a região crítica estiver livre, a thread que chamou o procedimento continua sua execução acessando a região crítica. Caso a região crítica estiver ocupada, a thread que chamou o mutex_lock ficará bloqueada até que a thread que está na região crítica termine sua execução e chame o procedimento mutex_unlock, liberando o recurso e permitindo a execução da thread que estava bloqueada. 
- Monitores (Hansen, 1973; Hoare, 1974): são mecanismos de alto nível para garantir sincronização e exclusão mútua, implementados pelo próprio compilador. Processos não têm acesso à estrutura interna do monitor apenas utilizam chamadas a seus procedimentos. A exclusão mútua é garantida pelo compilador e o programador só precisa especificar quais as regiões críticas. Em um monitor apenas um processo pode estar ativo num dado momento e se outro processo tentar acessá-lo, ele precisa ser bloqueado. Uma maneira de realizar o bloqueio é por meio da utilização de variáveis de condição, que permitem duas operações (wait e signal). Quando um procedimento não pode prosseguir, ele emite um wait sobre uma variável condicional e isso bloqueia o processo que está chamando, permitindo que o outro processo proibido de entrar no monitor entre. Esse outro processo pode acordar o parceiro adormecido a partir da emissão de um signal para a variável condicional do parceiro que está esperando. A utilização de variáveis de condição proporciona uma sincronização condicional (Tanenbaum, 1995).

O suporte às primitivas necessárias ao desenvolvimento de programas concorrentes é obtido com a utilização de uma linguagem de programação que garanta a divisão do problema de maneira justa entre os processadores e a execução concorrente dos processos (leves ou não). Além de linguagens de programação propriamente ditas, o paralelismo pode ser implementado através de bibliotecas que associam uma nova API (conjunto de rotinas para programação de aplicações que não envolvem detalhes de implementação) às linguagens sequenciais, estendendo suas funcionalidades para atender aos requisitos da programação concorrente. As principais ferramentas de suporte à programação concorrente são definidas de acordo com a arquitetura de memória utilizada, que pode ser compartilhada ou distribuída. No contexto de memória distribuída o principal ambiente de programação usado é o MPI (Message Passing Interface) (Lastovetsky, 2003). No contexto de memória compartilhada os principais modelos usados são o OpenMP e o Padrão Posix para Threads (Andrews, 1991; Grama et al., 2003; Quinn, 2003). Devido à importância do padrão PThreads para este projeto, o mesmo será detalhado a seguir.

\subsubsection{Pthreads - API POSIX para Threads}

Empresas de hardware e software têm desenvolvido API próprias para o uso de threads para programação concorrente usando o paradigma de memória compartilhada. Algumas dessas APIs, são: NT threads, Solaris, Java threads, entre outras (Grama et al., 2003). Embora sejam semelhantes, pois são baseadas no modelo multithreading, apresentam variações e incompatibilidades. Assim, dentre essas, a que merece maior destaque é a 
Pthreads, pois foi estabelecida como padrão pelo IEEE Std 1003.1c-1995 (POSIX, 2003) passando a ser suportada por diversos fornecedores.

PThreads são definidas sobre a linguagem $\mathrm{C}$ através de chamadas de procedimentos, por meio de implementações da biblioteca pthread.

O programa hello.c mostrado na Figura 2.1 exemplifica o uso das funções de criação inicialização, sincronização e finalização de threads do padrão PThreads.

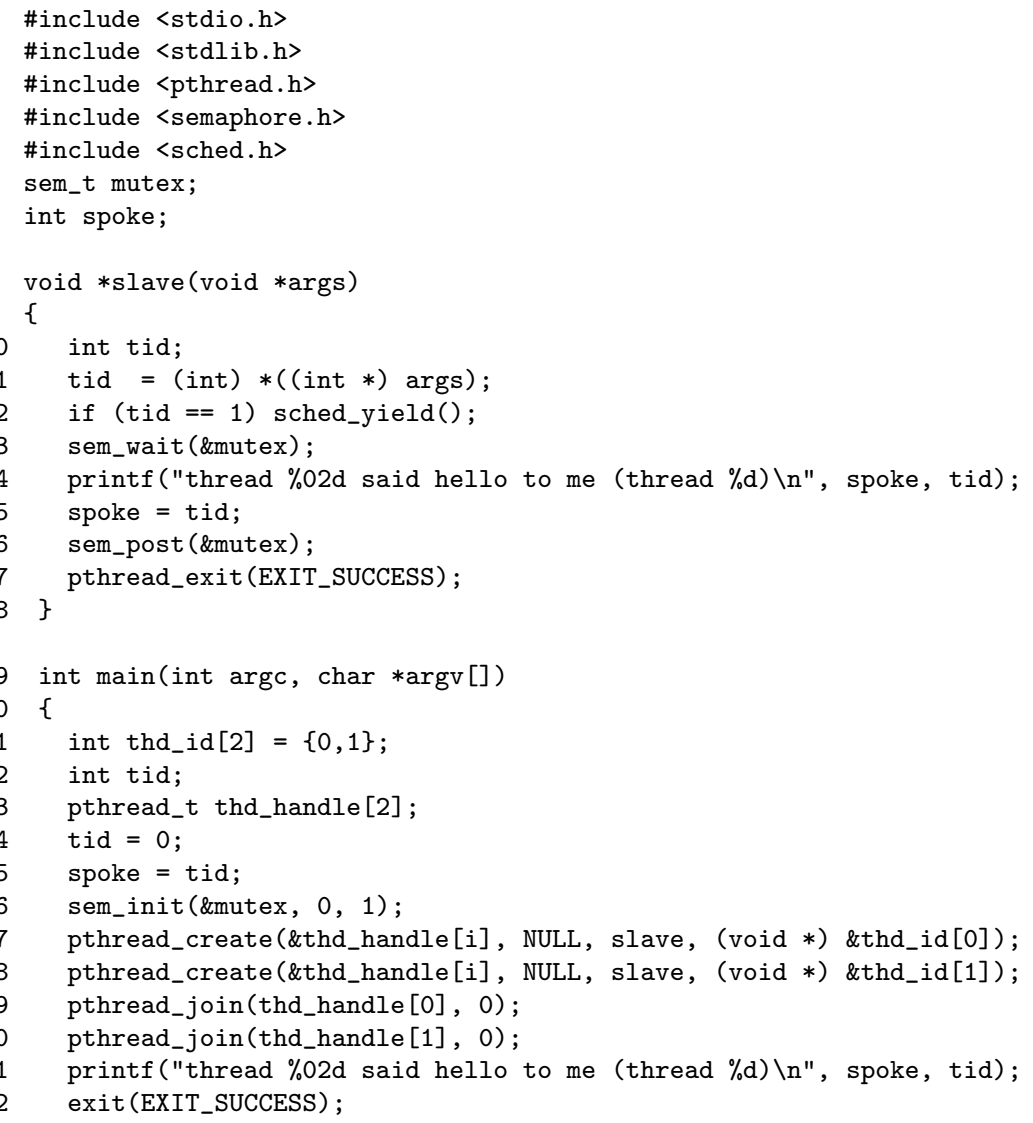

Figura 2.1: Código fonte do programa hello.c implementado usando o padrão PThreads.

Nesse programa, nas linhas 27 e 28 são criadas duas threads por meio da função pthread_create(). Essa função cria threads simples com um único identificador e as deixa prontas para serem executadas. A thread criada pode ser chamada em qualquer parte do programa e a quantidade de threads criadas depende da implementação. Nesse programa as threads são responsáveis por executar a função slave(). Na função slave() o id da thread é analisado, se for igual a 1, a thread tem sua execução suspensa pela função sched_yeld() na linha 12. Isso ocorre porque a thread com $i d=1$ é a thread mestre, que coordena as duas outras threads escravas. Se o id da thread for diferente de 1, a thread 
continua sua execução e apresenta na tela uma mensagem de texto, a qual é impressa de forma síncrona, ou seja, uma thread por vez e essa sincronização é obtida pelo uso de um semáforo (variável mutex). Na linha 6 do programa o semáforo mutex é definido, e inicializado na linha 26. Esse semáforo garante a exclusão mútua da região crítica por meio das primitivas wait(bloqueio da thread) na linha 13 e post(liberação da thread) na linha 16. A região crítica compreende a função que retorna uma mensagem de texto e utiliza a variável compartilhada spoke. A função pthread_exit() na linha 17 finaliza a execução de cada uma das threads. Por fim, a função pthread_join(), nas linhas 29 e 30, garante a sincronização da finalização das threads. A thread mestre que estava suspensa pela função sched_yeld() retorna sua mensagem e o programa é finalizado.

\subsection{Teste Estrutural de Programas Concorrentes}

O projeto e o desenvolvimento de programas concorrentes são mais complexos se comparados com programas sequenciais. O projetista de aplicações concorrentes deve considerar tudo que se aplica a programas sequenciais, além dos aspectos específicos da programação concorrente. Entre os principais desafios da programação concorrente é possível citar (Sarmanho, 2009):

1. definir como o programa será dividido e quando será dividido;

2. planejar e implementar a interação entre os processos;

3. definir plataforma de hardware;

4. definir os aspectos de softwares a serem utilizados para o desenvolvimento e a execução;

5. traduzir todos os 4 elementos acima em speedup ${ }^{1}$. É possível que um programa concorrente mal projetado apresente desempenho pior que sua versão sequencial;

6. testar e depurar esse tipo de programa.

O teste de programas concorrentes é uma tarefa difícil, porém é fundamental que programas concorrentes apresentem alta qualidade e estejam livres de defeitos. Por este motivo a aplicação da atividade de teste faz-se necessária, assim como a definição de critérios que auxiliem a etapa de geração e avaliação de casos de teste, oferecendo medidas de cobertura que melhoram a qualidade dos testes. Nesse contexto, esforços têm sido

\footnotetext{
${ }^{1}$ Cálculo relacionado à vantagem em utilizar paralelismo para resolução de um problema complexo. Definido pela razão tempo de execução da versão sequencial pelo tempo de execução da versão concorrente do programa (Dongarra et al., 2003; Grama et al., 2003).
} 
conduzidos com o intuito de definir critérios de teste considerando aspectos específicos relacionados à concorrência.

Uma das linhas de pesquisa do grupo de pesquisa em Engenharia de Software do ICMC/USP é relacionada ao teste de programas concorrentes. Nessa linha o grupo tem pesquisado o mapeamento de critérios de teste para esse contexto, considerando informações sobre o fluxo de controle, dados, comunicação e sincronização desses programas.

As pesquisas iniciaram com a definição de critérios estruturais para programas com memória distribuída. Um modelo de teste baseado no fluxo de controle e no fluxo de dados e comunicação de programas paralelos é estabelecido para extrair informações relevantes na realização dos testes (Souza et al., 2005). Esse modelo é baseado no trabalho de Yang et al. (1998), sendo composto por um grafo denominado PCFG (Parallel Control Flow Graph) para o contexto de programas com passagem de mensagens. Baseados nesse modelo foram propostos critérios de teste específicos para programas concorrentes no contexto de passagem de mensagens.

Sarmanho (2009) propõe um novo modelo o PCFGsm (Parallel Control Flow Graph for Shared Memory) baseado no modelo PCFG anterior estabelecendo critérios de teste estrutural para programas que utilizam o paradigma de memória compartilhada. Esse novo modelo suporta: a análise estática do programa, a geração dos elementos requeridos utilizando os critérios de teste propostos em Souza et al. (2008a) e adaptados para o contexto de memória compartilhada e um método que registra a comunicação entre threads por meio das sincronizações realizadas (Sarmanho, 2009). O PCFGsm é proposto com o intuito de capturar as informações necessárias para a cobertura dos critérios de teste.

Foi necessário definir um novo modelo para o contexto de memória compartilhada, pois o PCFG não se adaptava às características desses programas. Nesses programas há a necessidade de diferenciar a comunicação e a sincronização entre os processos (ou threads). Além disso, as variáveis compartilhadas fazem parte do mesmo espaço de endereçamento, entretanto, devido ao não determinismo, não é possível determinar antes da execução os pares entre definição e uso de variáveis. Desse modo, informações sobre o fluxo de dados do programa não podem ser obtidas estaticamente, da forma que é feita no modelo anterior. O grafo PCFGsm é definido por:

- $\boldsymbol{n}$ : o número de threads geradas para o programa;

- comunicação: realizada por variáveis compartilhadas, comunicação implícita;

- sincronização: realizada por semáforos, sincronização explícita; e

- inicialização e finalização de threads: realizadas por meio de semáforos. 
Um programa concorrente $M$ é composto por $n$ threads $t^{i}$ de um processo $i$. As threads compartilham o mesmo espaço de endereçamento na memória. Cada thread possui um GFC $^{t}$ próprio análogo ao GFC de programas sequenciais. Esse GFC $^{t}$ é formado por um conjunto de nós $N^{t}$ e um conjunto de arestas $E^{t}$. Nós pertencentes a uma mesma thread são representados por: $n_{i}^{t} \in N^{t}$.

Na figura 2.2 é ilustrado o PCFGsm para o programa hello.c, apresentado na Seção 2.3. O grafo mostra a thread main e as duas threads que executam a função slave, representadas por t0, t1 e t2, respectivamente. No grafo são apresentadas informações de definição e uso de variáveis e arestas tracejadas representam arestas de sincronização.

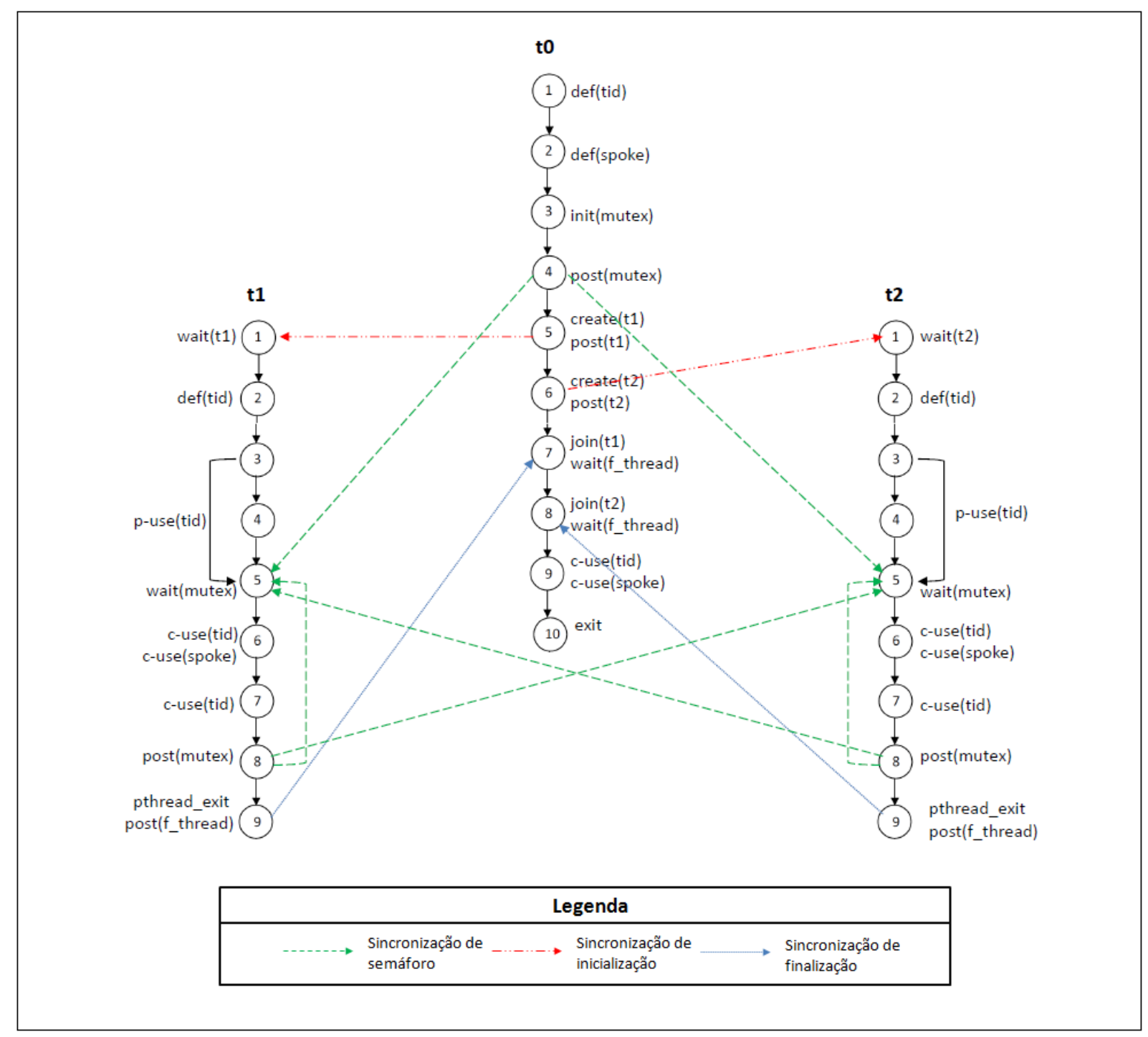

Figura 2.2: PCFGsm para o programa hello.c.

A sincronização entre as threads é garantida através de primitivas post e wait, semelhantes as primitivas send e recv presentes no contexto de passagem de mensagem, porém wait corresponde a causa e o post o efeito em uma relação causa-e-efeito. No grafo as 
sincronizações são representadas por arestas que partem de um nó que contém uma instrução post para outro que contém a instrução wait, as arestas podem ser de dois tipos: inter-threads se sincronizam duas threads diferentes ou intra-thread que ligam nós de uma mesma thread. Arestas dentro de uma mesma thread podem ser do tipo intra-thread se na thread a sincronização com primitivas post e wait referem-se ao mesmo semáforo.

As informações sobre o fluxo de dados são extraídas dinamicamente, pois a comunicação acontece de forma implícita por meio de operações de escrita e leitura na variável compartilhada. Tendo como base o conceito de timestamp, definido por Lei e Carver (2006), a comunicação entre as threads é determinada com a utilização de timestamps (rótulos de tempo). A atribuição de timestamps é feita seguindo o trace de execução do programa, possibilitando determinar a ordem dos eventos concorrentes e estabelecer os pares de comunicação.

Os nós $N$ que compõem o PCFGsm podem ser de três tipos distintos: nós que pertencem a uma thread $N^{t}$, nós que estão associados a primitiva de comunicação wait $N^{w}$ e nós associados a primitiva post $N^{p}$.

As variáveis $V$ definidas para programas concorrentes com memória compartilhada podem ser de três tipos distintos: variáveis "virtuais" $V_{S}^{t} \subset V$ que representam a sincronização referente à inicialização e finalização das threads, variáveis locais $V_{L}^{t} \subset V$ que são as variáveis privadas da thread e variáveis compartilhadas $V_{C}^{t} \subset V$ que possuem acesso global. Os seguintes tipos de usos são definidos para as variáveis:

- c-use: uso computacional, quando há uma declaração de variável em um nó $n_{i}^{p}$ do grafo.

- p-use: uso predicativo, está associado a uma condição associada a comando de fluxo de de controle em arestas intra-processos $\left(n_{i}^{p}, n^{p}\right)$ do grafo.

- sync-use: uso de sincronização, quando a variável $x$ está associada a instruções de sincronização, a variável $x$ é do tipo semáforo $x \in V_{S}$;

- comm-c-use: c-uso comunicacional, ocorre quando há um uso computacional de uma variável compartilhada;

- comm-p-use: p-uso comunicacional, ocorre quando há um uso predicativo de uma variável compartilhada.

Esse modelo possui as seguintes associações:

- associação c-use: representada por $\left(n_{i}^{p}, n_{j}^{p}, x\right)$ tal que $x \in \operatorname{def}\left(n_{i}^{p}\right)$, e $n_{j}^{p}$ contém um $c$-uso de $x$ e há um caminho livre de definição com relação à $x$ de $n_{i}^{p}$ para $n_{j}^{p}$. 
- associação p-use: representada por $\left(n_{i}^{p},\left(n_{j}^{p}, n_{k}^{p}, x\right)\right.$ tal que $x \in \operatorname{def}\left(n_{i}^{p}\right)$, e $\left(n_{j}^{p}, n_{k}^{p}\right)$ contém um $p$-uso de $x$ e há um caminho livre de definição com relação a $x$ de $n_{i}^{p}$ para $\left(n_{j}^{p}, n_{k}^{p}\right)$.

- associação sync-use: representada por $\left(n_{i}^{t},\left(n_{j}^{t}, n_{k}^{q}\right), s\right)$ se e somente se $s \in V_{S}$, $\left(n_{j}^{t}, n_{k}^{q}\right)$ possui um sync-use de $s$ e existe um caminho livre de definição em relação a $s$ de $\left(n_{i}^{t}\right)$ até a aresta $\left(n_{j}^{t}, n_{k}^{q}\right)$.

- associação comm-c-use: representada por $\left(n_{i}^{t}, n_{j}^{q}, x\right)$ se e somente se $x \in V_{C}, x \in$ $\operatorname{def}\left(n_{i}^{t}\right)$, e $n_{j}^{q}$ contém um c-uso de $x$, onde $x$ é uma variável compartilhada.

- associação comm-p-use: representada por $\left(n_{i}^{t},\left(n_{j}^{q}, n_{k}^{q}, x\right)\right.$ se e somente se $x \in V_{C}$, $x \in \operatorname{def}\left(n_{i}^{t}\right),\left(n_{j}^{q}, n_{k}^{q}\right)$ contém um $p$-uso da variável compartilhada $x$.

Com base no modelo proposto, no fluxo de dados, de controle e de comunicação, bem como as definições são propostos dois conjuntos de critérios de teste estrutural para programas paralelos com memória compartilhada (Sarmanho, 2009):

1. Critérios baseados no fluxo de controle:

- All-p-nodes: requer a execução de todos os nós post $n_{i}^{t} \in N_{p}$;

- All-w-nodes: requer a execução de todos os nós wait $n_{i}^{t} \in N_{w}$;

- All-nodes: requer a execução de todos os nós $n_{i}^{t} \in N$;

- All-s-edges: requer a execução de todas as arestas de $\operatorname{sincronização~}\left(n_{i}^{t}, n_{j}^{q}\right)$ $\in E_{S} ; \mathrm{e}$

- All-edges: requer a execução de todas as arestas $\left(n_{i}^{t}, n_{j}^{q}\right) \in E$.

2. Critérios baseado no fluxo de dados e comunicação:

- All-defs-comm: requer a execução de caminhos que executem ao menos uma associação comm-c-use ou comm-p-use para cada ponto de definição da variável $x \in V_{c}$;

- All-defs: requer a execução de caminhos que executem ao menos uma associação c-use, p-use, comm-c-use ou comm-p-use para cada ponto e definição da variável $x \in \operatorname{def}\left(n_{i}^{t}\right)$;

- All-comm-c-uses: requer a execução de caminhos que executem todas as associações comm-c-use;

- All-com-p-use: requer a execução de caminhos que executem todas as associações comm-p-use; 
- All-c-uses: requer a execução de caminhos que executem associações c-use;

- All-p-uses: requer a execução de caminhos que executem associações p-use; e

- All-sync-uses: requer a execução de caminhos que executem todas as associações sync-use.

Com o objetivo de validar as ideias propostas anteriormente, ferramentas de apoio foram implementadas. Souza et al. (2005) apresentam a ferramenta denominada Valipar. Essa ferramenta representa uma arquitetura geral, da qual foram instanciadas diferentes versões para tratar diferentes tipos de programas concorrentes. A ValiPar foi instanciada inicialmente para PVM, ferramenta ValiPVM (Souza et al., 2008b) e para MPI, ferramenta ValiMPI (Hausen e Vergilio, 2005; Hausen et al., 2007). Endo et al. (2008) instancia a ValiPar para o teste de $C W S$ em BPEL definindo assim uma ferramenta web que automatiza o processo de teste chamada ValiBPel (Endo et al., 2008). Para programas concorrentes que utilizam o paradigma de memória compartilhada Sarmanho (2009) propõe a ferramenta ValiPThread que automatiza a aplicação do modelo PCFGsm descrito na seção anterior e possibilita a avaliação dos critérios de teste definidos tratando aspectos específico do paradigma de memória compartilhada para programas escritos em Pthread.

A ferramenta ValiPThread é composta por cinco módulos principais: Valipthread_inst, Valipthread_Elem, Valipthread_Eval, Valipthread_Sync e Valipthread_Exec (Figura 2.3). O módulo Valipthread_Inst é responsável por realizar a análise estática do programa paralelo. Recebe como entrada o programa paralelo a ser testado, realiza a instrumentação através da inserção de check-points (marcadores) do programa com base nas informações do GFC. O programa instrumentado é usado para gerar os caminhos percorridos no grafo e a sequência de sincronização obtida com a execução. O PCFG é gerado tendo como base as informações sobre nós e arestas, definição e uso de variáveis e uso de primitivas post e wait.

A definição dos elementos requeridos é realizada no módulo Valipthread_Elem. Os elementos requeridos para a cobertura dos critérios definidos são gerados com base no caminho percorrido no PCFG e das informações sobre o fluxo de dados gerados no módulo anterior. Esse módulo gera também descritores e grafos auxiliares (grafo(i) e grafo reduzido de herdeiros) os quais são utilizados pelo módulo Valipthread_Eval para verificar quais elementos requeridos foram cobertos pelos casos de teste executados.

O módulo Valipthread_Exec recebe como entrada o programa instrumentado no módulo Valipthread_Inst e os dados de teste fornecidos pelo usuário realizando a execução do programa. Durante a execução são gravadas as sequências de sincronização e o caminho percorrido por cada processo, criando um trace de execução. 


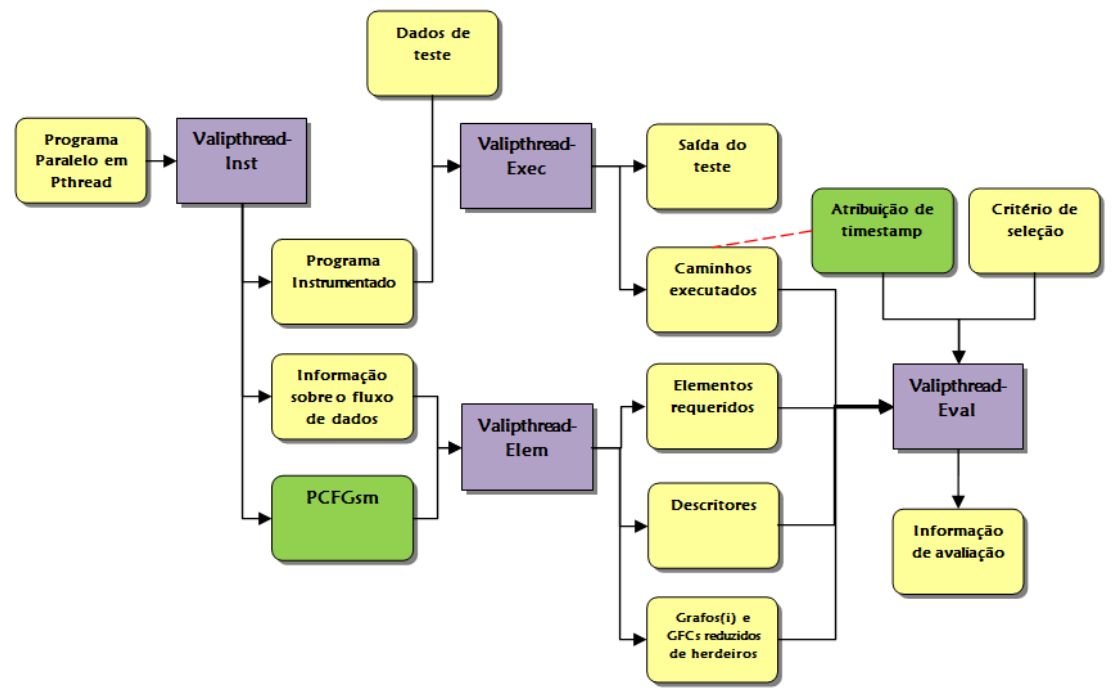

Figura 2.3: Arquitetura da ferramenta ValiPthread (Sarmanho, 2009).

O módulo Valipthread_Eval recebe o trace obtido no módulo Valipthread_Exec, os elementos requeridos e o grafo reduzido de herdeiros e realiza a avaliação da cobertura dos critérios definidos. Como saída, proporciona o percentual de cobertura e a lista de elementos cobertos pelo caso de teste.

O módulo Valipthread_Sync implementa o teste de alcançabilidade proposto por Lei e Carver (2006). A partir da execução de um caso de teste, usando informações sobre os traces gerados, esse módulo gera as possíveis variantes de sincronizações que são possíveis para esse caso de teste. Para execução das variantes, traces parciais são gerados, a fim de realizar a execução controlada dessas variantes. Detalhes desse módulo são encontrados em Camillo (2008).

A ValiPThread realiza o teste baseado no modelo PCFGsm que captura as informações necessárias ao teste, em quatro etapas:

1. análise estática: a partir do PCFGsm são obtidas as informações sobre a execução do programa, que serão utilizadas posteriormente no teste;

2. definição dos elementos requeridos: os elementos necessários à cobertura dos critérios são gerados com base no modelo de teste PCFGsm;

3. definição e execução dos casos de teste: um critério de cobertura é estabelecido e o programa em teste é executado com o caso de teste selecionado para a cobertura de um elemento requerido específico; e

4. análise de cobertura alcançada: a análise da cobertura é feita com base na taxa de cobertura conseguida pela aplicação do caso de teste sob um critério definido. Casos de teste são definidos e executados até que a taxa de cobertura desejada seja alcançada. 
Para ilustrar, a seguir é descrito como ocorre a execução do teste na ferramenta ValiPThread:

\section{Realização da análise estática e instrumentação pelo módulo Valipthread_} Inst:

Para execução do teste, será usado o programa hello.c, mostrado na Figura 2.1. Como entrada ao módulo Valipthread_Inst é necessário apenas passar como parâmetro o código fonte do programa, por meio da seguinte linha de comando:

\$valipthread_inst hello.c

Esse comando gera os seguintes arquivos:

- hello.c_09_instrumentado_final.c: contém o programa instrumentado;

- hvalipthread.h: contém as variáveis utilizadas pelo programa instrumentado, usadas apenas no programa original;

- c.main.dot: contém o GFC da função main em formato .dot;

- c.slave.dot: contém o GFC da função slave do programa em formato .dot;

- shared.var: contém as variáveis compartilhadas do programa, separadas pelo tipo, que pode ser de comunicação ou de sincronização; e

- thread.names: contém o nome das threads do programa, as threads representam as funções do programa. No caso do programa hello.c seriam as funções main e slave.

\section{Definição dos elementos requeridos pelo módulo Valipthread_Elem:}

O módulo Valipthread_Elem recebe como entrada o PCFGsm e as informações de fluxo de dados que foram gerados pelo módulo Valipthread_Inst. A linha de comando para execução do módulo é a seguinte:

\$ valipthread_elem 3 "main(0)" "slave(1,2)"

Na linha de comando acima, o argumento "3" corresponde ao número máximo de threads geradas durante a execução do programa, "main(0)" é o nome da thread que possui a função main identificada pelo número "0" e "slave(1,2)" corresponde a thread que corresponde a função slave e os argumentos "1" e "2" são os identificadores destas threads.

Esse comando gera todos os elementos requeridos para os critérios baseados no fluxo de controle, de dados e de sincronização definidos pelo modelo PCFGsm e seus respectivos descritores. Para obtenção dos elementos requeridos, há também 
a geração dos grafos(i), grafos reduzidos de herdeiros e descritores para todos os critérios. Como exemplo, pode-se citar o critério all-s-edges, que gera os arquivos:

- All-edgesS.req: a lista dos elementos requeridos pelo critério all-s-edges;

- All-edgesS.aut: contém os descritores para a cobertura dos elementos requeridos pelo critério all-s-edges. Descritores são expressões que descrevem um caminho que possibilita a cobertura de um elemento requerido.

A Figura 2.4 exibe o conteúdo do arquivo All-edgesS.req, onde cada linha contém um requisito que precisa ser exercitado. Para esse critério, um requisito corresponde a uma aresta de sincronização. Para representar essa aresta é inserido o número do nó onde ocorre um post ou wait seguido do número do processo. Assim, a aresta (5-0 5-1) corresponde a um post no nó cinco do processo zero e um wait no nó cinco do processo um.

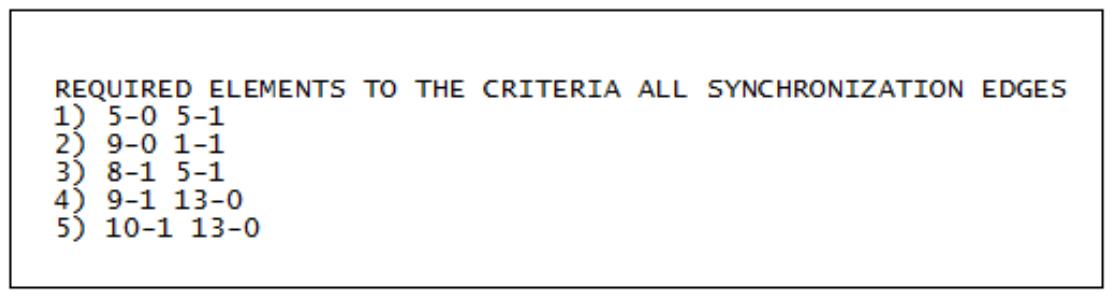

Figura 2.4: Conteúdo do arquivo All-edgesS.req.

Na Figura 2.5 é apresentado o conteúdo do arquivo All-edgesS.aut, onde cada linha corresponde a um descritor. Para o primeiro descritor, ou seja, para a primeira linha do arquivo All-edgesS.aut, na Figura 2.6 é explicada de maneira detalhada a maneira como o descritor deve ser interpretado. Neste caso, o elemento requerido [1) $\left.\begin{array}{lll}1 & 3 & 3\end{array}\right]$ a que o descritor se refere é o primeiro número da sequência, no caso o número 1 , os três valores seguintes 133 definem que o elemento requerido é formado por 1 autômato formado por 3 estados e que seu estado final é o estado 3. Em seguida, cada estado é especificado, para o estado 1 (1 1:Np1 2:5-0 0), onde o primeiro número indica o estado, 1 neste caso, a condição de transição do estado 1 para ele próprio é passar por qualquer nó do processo p1, a condição de transição do estado 1 para o estado 2 é passar pelo nó 5 do processo 0 . O número 0 indica o fim de um estado. Os estados seguintes 2 e 3 acompanham a mesma lógica de especificação. Todos os demais descritores são estruturados dessa forma.

Autômatos finitos são usados para validar um elemento requerido, ou seja, se o caminho descrito (elemento requerido) for aceito pelo autômato então o elemento requerido foi coberto. O autômato em modo gráfico que representa o descritor 1 , explicado acima é ilustrado na Figura 2.7. 


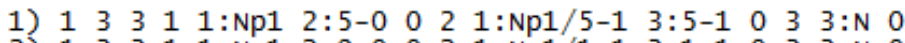

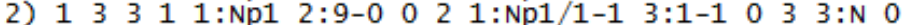

3) $\begin{array}{lllllllllllllllll}1 & 3 & 3 & 1 & 1: N p 1 & 2: 8-1 & 0 & 2 & 1: N p 1 / 5-1 & 3: 5-1 & 0 & 3 & 3: N & 0\end{array}$

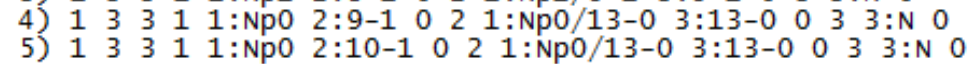

Figura 2.5: Conteúdo do arquivo All-edgesS.aut

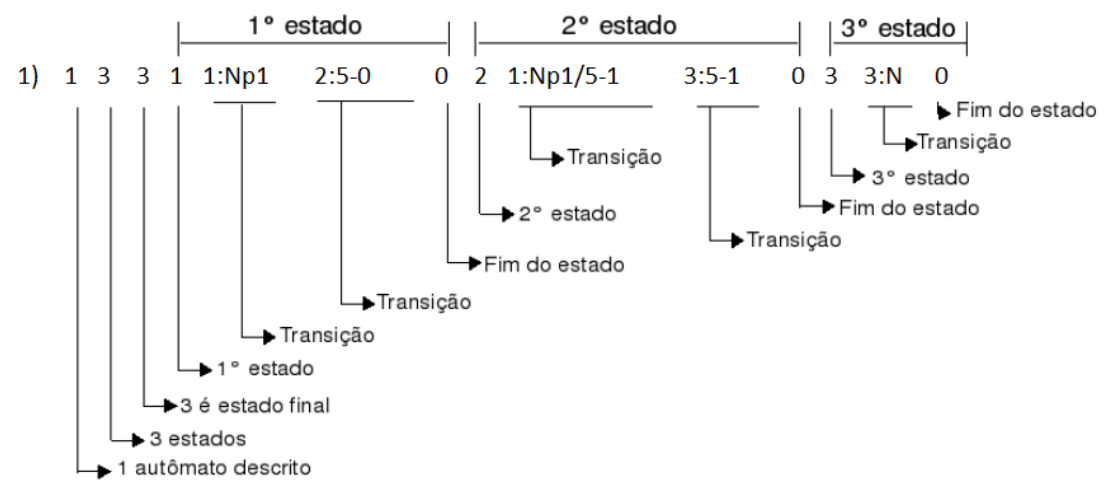

Figura 2.6: Interpretação do autômato em modo texto.

\section{Execução dos casos de teste pelo módulo Valipthread_Exec:}

O módulo Valipthread_Exec é responsável pelo apoio à execução dos casos de teste.

O primeiro passo é compilar o programa instrumentado. O script valipthread_cc compila o programa instrumentado hello.c_instrumentado_final com o gcc, o comando é:

\$valipthread_cc hello_inst hello.c_09_instrumentado_final.c

O passo seguinte é executar os casos de teste, cada caso de teste é executado separadamente com o comando:

\section{\$./valipthread_exec 1 run $3 . /$ hello.inst}

Onde: "1" é o número que identifica o caso de teste, "run" indica que para o caso de teste deseja-se obter o trace de sua execução não-determinística, "3" indica a quantidade máxima de threads a serem criadas "hello.inst" é o programa instrumentado. Nesse exemplo, o número "1" identifica o caso de teste definido pelo usuário, que poderá ser usado por outros módulos da ferramenta. Um caso de teste é formado pelos valores de entrada e a respectiva saída esperada para o programa em teste. Nesse caso o programa não necessita de nenhum parâmetro de entrada. A saída obtida pela execução do programa com o caso de teste 1 no módulo Valipthread_Exec é apresentada na Figura 2.8.

A execução do caso de teste gera também o trace de execução do programa. O trace de execução contém os nós que formam os caminhos percorridos em cada 


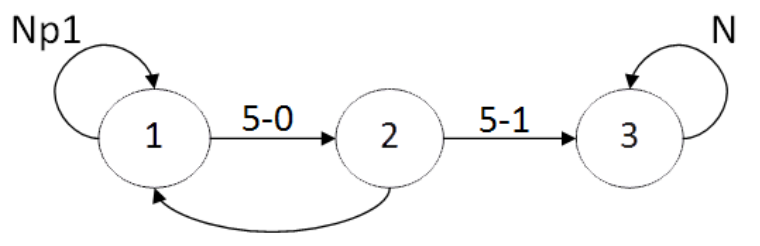

1:Np1/5-1

Figura 2.7: Autômato finito em modo gráfico.

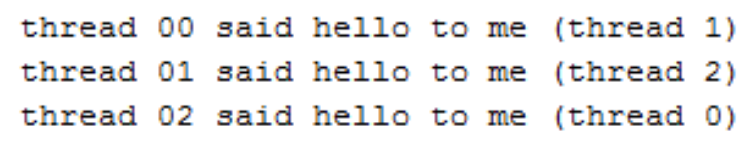

Figura 2.8: Saída do programa hello.c para o caso de teste 1.

thread com a execução do caso de teste. Os traces serão usados posteriormente para avaliar quais elementos requeridos foram cobertos por um dado critério de teste no módulo Valipthread_Eval. Na Figura 2.9 é mostrado os traces de execução de cada uma das threads que compõem o programa hello.c.

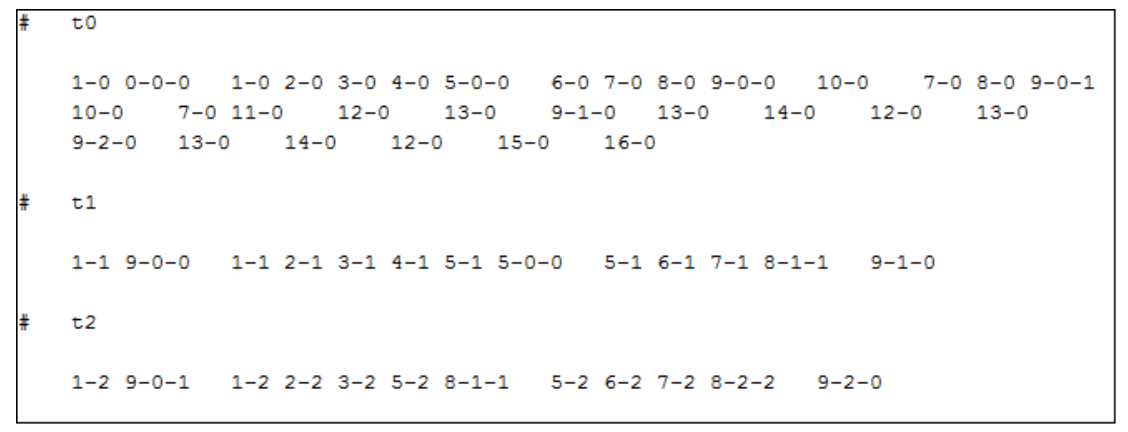

Figura 2.9: Traces de execução do programa hello.c para o caso de teste 1.

O resultado final da execução do módulo Valipthread_Exec é um subdiretório com informações sobre o caso de teste executado, com: o trace de execução das threads, a ordem de eventos de sincronização executados para cada semáforo, o arquivo "args" com o registo das entradas fornecidas, o arquivo "stdout" com o registo da saída obtida pela execução do programa, o arquivo "stdin" o registro do que foi fornecido pelo usuário como entrada padrão e o arquivo "nthreads" com o número de threads criadas e ou executadas pelo programa. Para a execução do programa hello.c os arquivos criados são: mem_trace.mainto, mem_trace.slave.t1, mem_trace.slave.t2, nthreads, stderr, stdout, trace.main.t0, trace.slave.t1 e trace.slave.t2. 


\section{Avaliação da cobertura dos critérios pelo módulo valipthread_Eval:}

O módulo Valipthread_Eval é responsável por avaliar a cobertura dos critérios de teste. Recebe como entrada as informações sobre o caso de teste executado pelo Valipthread_Exec, juntamente com o caso de teste escolhido pelo usuário e os respectivos descritores e elementos requeridos. Após a execução do módulo, o resultado da avaliação da cobertura do critério é retornado ao usuário.

A execução do módulo é feita pela seguinte linha de comando:

\$valipthread_eval all-edgesS 3 "main(0)" "slave(1,2)"

No qual: "all-edgesS" é o critério que se deseja cobrir, "马" é o número máximo de threads a serem criadas e os dois últimos argumentos são as threads com os parâmetros que correspondem aos seus identificadores.

O resultado da execução do módulo Valipthread_Eval com a taxa de cobertura atingida pela execução do caso de teste 1 para o critério all-s-edges para o programa hello.c é apresentado na Figura 2.10.

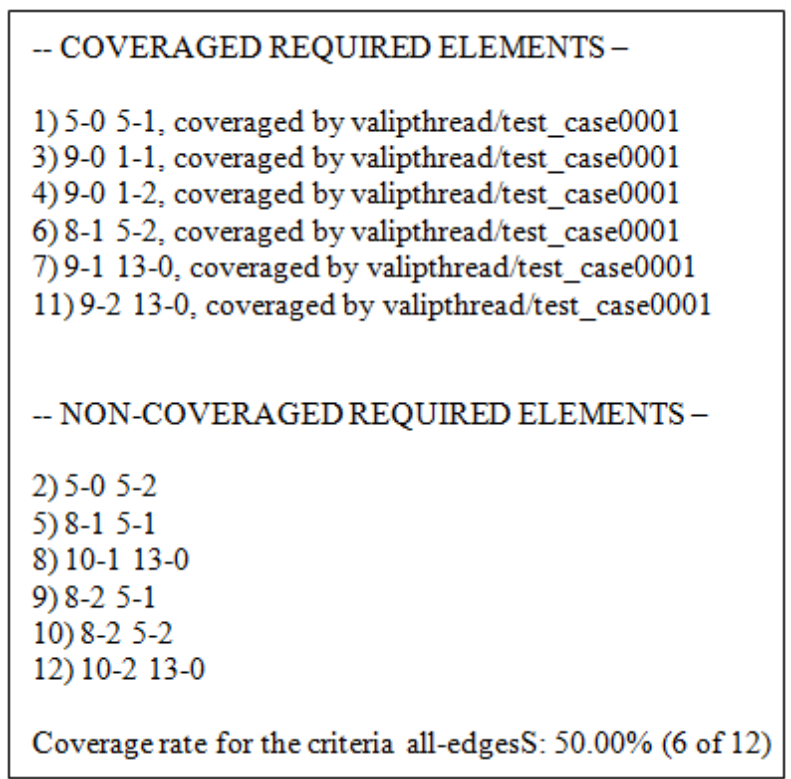

Figura 2.10: Resultado da cobertura do critério all-s-edges.

\subsubsection{Experimentação em Teste de Programas Concorrentes}

Embora critérios tenham sido definidos assim como modelos que apoiam o uso destes critérios, esses estudos ainda são bastante incipientes, pois não disponibilizam estudos experimentais que possibilitem sua replicação em outros contextos, o que proporcionaria uma avaliação mais eficaz de sua viabilidade. 
Visando dar continuidade aos trabalhos do grupo de pesquisa do ICMC, Brito (2011) propõe um estudo experimental para avaliar aspectos de custo e eficácia em revelar defeitos dos critérios de teste definidos para programas concorrentes que utilizam o paradigma de passagem de mensagem. Esse estudo busca definir melhorias aos critérios a fim de torná-los mais eficientes, estabelecendo estratégias de baixo custo na detecção de erros.

Uma revisão sistemática (Brito, 2011) foi realizada com o propósito de reunir trabalhos relacionados à atividade de teste em programas concorrentes, utilizados como base teórica, além de fonte de conhecimento sobre demais critérios existentes que possam ser analisados e talvez incorporados ao trabalho. Devido ao estudo experimental envolver análise de eficácia, essa revisão buscou também trabalhos que classificassem defeitos em programas concorrentes.

Após analisar os trabalhos relacionados à classificação de defeitos, foi definido um conjunto de defeitos que foram semeados nos programas considerados no experimento. Para isso, foi feito um estudo sobre como semear defeitos nesse tipo de programa.

Com relação aos programas utilizados no experimento, foram utilizados os programas empregados no trabalho de Machado (2008). Além desses programas, foi utilizada também uma aplicação real, desenvolvida para a área de bioinformática.

Esse estudo fornece informações sobre a eficácia e o custo dos critérios de teste para programas concorrentes com passagem de mensagens. Algumas ideias são aproveitadas para o estabelecimento do experimento proposto neste trabalho.

Para avaliar as técnicas, métodos ou critérios é fundamental a realização de estudos experimentais. Estudos experimentais sobre teste de software em programas concorrentes ainda são incipientes, porém são fundamentais para o estabelecimento de estratégias de aplicação de critérios de modo a aproveitar as vantagens de cada um, para que possam ser avaliados por diferentes pessoas em diferentes ambientes, permitindo caracterizar de modo mais significativo os resultados obtidos (Souza et al., 2008a).

No contexto de teste de programas concorrentes, alguns estudos experimentais têm sido conduzidos a fim de comprovar a validade de propostas (Brito, 2011). A seguir são descritos alguns estudos relacionados a aplicação de estudos experimentais na avaliação de técnicas e ferramentas de teste para análise de programas concorrentes com memória compartilhada.

Um estudo experimental é conduzido por (Seo et al., 2006) a fim de comprovar a validade de sua proposta, que apresenta o teste baseado em especificação de programas concorrentes usando statecharts. O objetivo da proposta é diminuir o custo da atividade de teste usando a geração de interleavings representativos por meio de informações sobre o acontecimento de eventos, que representam sequências de sincronização, eliminando interleavings equivalentes. 
Joshi et al. (2009) propõe um framework denominado Callfuzer para teste ativo de programas concorrentes escritos em Java. Teste ativo é realizado em duas fases, primeiramente é efetuada uma análise dinâmica preditiva do programa a fim de detectar potenciais erros de concorrência, como condições de disputa, deadlock e violações de atomicidade. Na próxima fase os resultados da fase anterior são usados para controlar a execução do programa por intermédio do escalonador a fim de comprovar se estes erros são reais. Para cada tipo de erro foram implementados três detectores: RaceFuzzer para detecção de condições de corrida, AtomFuzzer para violações de atomicidade e DeadlockFuzzer para deadlock. Um estudo experimental foi conduzido ao término do desenvolvimento do projeto, o qual indicou que os detectores são eficientes para revelar defeitos previstos em programas concorrentes.

No trabalho de Mamun et al. (2010) é realizado um estudo experimental com o intuito de avaliar a eficácia das ferramentas de análise estática Coverity, Jtest, FindBugs e JSlint, quanto a sua capacidade de detectar erros de concorrência e padrões de erros para programas em linguagem Java, usando benchmarks de programas. Além disso, realiza um estudo comparativo entre ferramentas comerciais e ferramentas open source, que mostra que a eficácia das ferramentas varia de acordo com a categoria de erro analisada, provando que as ferramentas comerciais podem ser melhores na detecção de um tipo de erro, porém pior em relação a outro.

Propondo um estudo experimental para avaliação de conjuntos de critérios de teste, Saini (2005) reúne diversos esforços na definição de critérios de cobertura para programas concorrentes. Fazem parte desse estudo os critérios de teste estrutural all-synchronizationpair, all-branches, all-decision/condition, all-du-pair, all-synchronization-pair \& all-branches, all-syncronization-pair \& all decision/condition e all-synchronization-pair \& all-dupair. O critério de análise de mutantes é utilizado na inserção de defeitos e uma análise da eficácia em revelar defeitos é realizada com base na quantidade de mutantes mortos com a cobertura dos critérios de teste. Os resultados mostram que o critério all-synchronizationpair é mais custoso, o critério all-branches é mais robusto que all-syncronization-pair porém mais custoso, o critério all-decision/condition está na média entre todos os critério em termos de custo e robustez, o par de critérios all-synchronization-pair \& all-du-pair são os mais robustos entre os critérios avaliados no experimento e também os mais custosos, e o par all-synchronization-pair \& all-branches são os critérios mais vantajosos levando em consideração a relação robustez e custo dos critérios analisados.

Cordeiro e Fischer (2011) avaliam três abordagens para verificação de modelo de software multi-thread usando a teoria de satisfatibilidade de modelo para restringir o acesso e as primitivas de sincronização da biblioteca Pthreads. As três abordagens para verificação de modelo (Schedule Recording, Lazy e a abordagem UW) são implementadas em uma 
ferramenta chamada ESBMC. A ferramenta ESBMC é comparada com duas ferramentas de verificação de modelo amplamente utilizadas, a CHESS e a SATABS por meio de um estudo experimental. São utilizados diversos benchmarks para a avaliação neste trabalho, alguns destes benchmarks são utilizados também em nossos estudos por fornecerem uma variedade de programas em $\mathrm{C} / \mathrm{Phreads}$ com erros inseridos.

Embora alguns trabalhos apresentem estudos que comparam técnicas de teste voltadas a aplicações concorrentes, nenhum trabalho foi encontrado que compara técnicas usando os conceitos da Engenharia de Software Experimental e que considera os pontos básicos de comparação empírica: custo, eficácia e strength. Este trabalho visa contribuir nesse sentido desenvolvendo um estudo experimental para avaliar a efetividade de critérios de teste estruturais para programas concorrentes implementados usando o padrão Pthreads

\subsection{Considerações Finais}

Neste capítulo foi apresentada uma introdução ao teste de software e uma visão geral sobre a programação concorrente, foi abordado também o teste de programas concorrentes, priorizando a aplicação de técnicas de teste estrutural no contexto de programas concorrentes. Foram apresentados os principais esforços na condução de estudos experimentais para avaliação de propostas no contexto de teste de aplicações concorrentes.

O desenvolvimento deste capítulo objetivou contextualizar a área de teste de software e também o teste de programas concorrentes, evidenciando os objetos de estudo desta proposta. Esses conceitos são importantes para a compreensão dos capítulos subsequentes. 



\section{Definição, Planejamento e Operação do Experimento}

\subsection{Considerações Iniciais}

Este capítulo descreve as etapas iniciais do processo experimental. A primeira etapa inclui a definição do estudo experimental, seus objetos e os objetivos a serem alcançados. A próxima etapa compreende o planejamento do experimento, nessa fase o projeto do experimento é traçado, o processo de instrumentação é proposto e as ameaças a validade são avaliadas. Na etapa de operação do experimento são descritos o processo de execução do experimento, a maneira como o ambiente será preparado e quais artefatos serão utilizados. Os dados coletados e a maneira como serão avaliados a fim de provar as hipóteses são também estabelecidas durante essa fase. As demais etapas do processo experimental são definidas nos capítulos posteriores.

Na Seção 3.2 o experimento é definido em detalhes. A Seção 3.3 apresenta o planejamento do experimento. A Seção 3.4 descreve o processo de preparação do experimento para execução e a execução do experimento, apresentando e alguns dados coletados nesta fase. Por fim, na Seção 3.5 são apresentadas algumas considerações a respeito dos assuntos abordados neste capítulo. 


\subsection{Definição do Experimento}

O estudo experimental realizado neste projeto de mestrado segue os princípios da Engenharia de Software Experimental e o modelo de processo experimental proposto por Wohlin (Wohlin et al., 2000). Nesse modelo um estudo experimental é dividido em diferentes etapas: 1. definição, 2. planejamento, 3. execução, 4. análise e interpretação dos resultados e 5 . apresentação e empacotamento.

\subsubsection{Objetivo}

O objetivo deste experimento é analisar os critérios de teste estruturais definidos para programas concorrentes com memória compartilhada com o propósito de avaliar a efetividade dos critérios em relação aos fatores: custo, eficácia e strength, sob o ponto de vista do pesquisador no contexto de alunos de Pós-Graduação que atuam como sujeitos no experimento, a fim de comprovar a aplicabilidade dos critérios.

\subsubsection{Objeto}

O objeto de estudo deste trabalho são os critérios de teste: all-nodes, all-p-nodes, all-w-nodes, all-edges, all-s-edges, all-comm-c-uses, all-comm-p-uses, all-c-uses, all-p-uses e all-sync-uses, definidos por Sarmanho et al. (2008). Esses critérios são aplicados a programas concorrentes com memória compartilhada, considerando o padrão PThreads.

\subsubsection{Foco Qualitativo}

O foco qualitativo é medir a eficácia em revelar defeitos, o custo de aplicação e a dificuldade de satisfação dos critérios. O foco qualitativo secundário é analisar esses fatores a fim de estabelecer uma estratégia de aplicação dos critérios de teste, considerando a relação de inclusão entre eles.

\subsubsection{Perspectiva}

O experimento é avaliado em ambiente acadêmico.

\subsubsection{Contexto}

Este é um experimento desenvolvido em ambiente acadêmico, conduzido por alunos de Pós-Graduação do Instituto de Ciências Matemáticas e de Computação (ICMC) da Universidade de São Paulo, que atuam como sujeitos do experimento. Os sujeitos são res- 
ponsáveis por inserir defeitos nos programas a serem usados na avaliação dos critérios de teste. A experiência dos sujeitos é considerada, balanceada em relação aos assuntos que envolvem a execução do experimento. Para evitar um possível viés no estudo, uma entrevista prévia sobre o conhecimento dos alunos foi realizada para que os sujeitos fossem corretamente classificados de acordo com seu nível de conhecimento.

A aluna de mestrado que desenvolve este trabalho é responsável pelas demais fases que envolvem a condução do experimento. O estudo é conduzido como um estudo blocado nos objetos, com um único sujeito avaliando mais de um objeto. O sujeito usa todos os tratamentos, um objeto para todos os tratamentos e a ordem de uso é aleatória.

\subsection{Planejamento}

A fase de planejamento do experimento tem o objetivo de gerar um documento de planejamento que contenha todos os passos a serem seguidos para que o experimento seja conduzido de maneira adequada, a fim de atingir seus objetivos. Esta fase pode ser dividida em seis etapas, conforme as subseções seguintes (Wohlin et al., 2000).

\subsubsection{Seleção do Contexto}

Nesta etapa é definido o ambiente no qual o experimento será executado. O contexto desse experimento compreende o ambiente acadêmico, onde é realizada a avaliação dos critérios de teste estrutural para programas concorrentes com memória compartilhada propostos por Sarmanho et al. (2008).

Para avaliar os critérios foram utilizados programas selecionados dos benchmarks Inspect (Yang, 2011), Helgrind (Valgrind-Developers, 2012) e Rungta (Rungta e Mercer, 2009). Foram utilizados também cinco programas que implementam soluções para problemas clássicos da programação concorrente. Esses benchmarks são amplamente usados por pesquisadores da área de teste de programas concorrentes para avaliação de técnicas e novas metodologias (Cordeiro, 2010; Cordeiro e Fischer, 2011; Deniz et al., 2012; Mühlenfeld e Wotawa, 2007; Teixeira et al., 2010; Yang, 2009). Optou-se por utilizar programas provenientes de benchmarks já difundidos ao invés do desenvolvimento de novos programas exclusivamente para este experimento, pois acredita-se que, como já foram utilizados por outros pesquisadores, são representativos da população e também propiciam a comparação com as demais teorias propostas, além de não inserir viés, projetando programas que atendem aos requisitos dos critérios de teste avaliados.

O estudo contou com 13 sujeitos, classificados em dois tipos distintos: alunos de pós-graduação, que atuam como semeadores de defeitos por meio da inserção de defeitos nos programas selecionados e a condutora do experimento, responsável pela elaboração 
de casos de teste para cobertura de elementos requeridos, usando a ferramenta de teste ValiPThread para auxiliar na atividade de teste.

Uma taxonomia de defeitos para programas concorrentes em PThreads, foi utilizada para classificar os defeitos inseridos pelos sujeitos de modo a verificar se todos os tipos de defeitos propostos na taxonomia haviam sido considerados. A utilização de programas desenvolvidos por terceiros e a seleção de sujeitos diferentes para a tarefa de inserção de defeitos visa mitigar o viés de inserir defeitos conforme conhecimento dos critérios de teste analisados.

\subsubsection{Definição das Hipóteses}

Com base na definição do experimento é derivada a seguinte questão a ser respondida com a realização deste experimento:

Qual a eficácia, custo e aspecto complementar dos critérios de teste estruturais definidos para programas concorrentes com memória compartilhada?

Buscando responder esta questão por meio da estatística descritiva, as seguintes hipóteses foram formalizadas:

- HN1 - Os critérios de teste estrutural definidos para programas concorrentes não são significativamente diferentes em relação ao custo de aplicação.

- HA1 - Os critérios de teste estrutural definidos para programas concorrentes apresentam custo de aplicação significativamente diferente.

- HN2 - Os critérios de teste estrutural definidos para programas concorrentes não são significativamente diferentes em relação a eficácia em revelar defeitos.

- HA2 - Os critérios de teste estrutural definidos para programas concorrentes apresentam eficácia em revelar defeitos significativamente diferente.

- HN3 - Não há relação de inclusão entre os critérios avaliados.

- HA3 - Há alguma relação de inclusão entre os critérios avaliados.

\subsubsection{Seleção dos Sujeitos}

A seleção dos indivíduos que farão parte do experimento foi feita por conveniência, selecionando pessoas com conhecimento em desenvolvimento de programas concorrentes. No caso desse estudo, em especial, por se tratar de um assunto complexo, de difícil manipulação, que exige conhecimento multidisciplinar e específico em diferentes áreas de conhecimento, foram selecionados de maneira aleatória alunos de mestrado do ICMC/USP que já tinham 
concluído a disciplina de programação concorrente. A conclusão dessa disciplina é importante, pois é necessário que os alunos possuam um conhecimento razoável que possibilite a compreensão dos programas que lhe são atribuídos e formulação de novos programas com erros inseridos. A fim de nivelar o conhecimento dos alunos, os programas são distribuídos conforme o grau de experiência declarado pelos mesmos em relação a cada uma das áreas envolvidas, a medida que o conhecimento aumenta programas mais complexos são atribuídos. Todas as demais fases do processo experimental são conduzidas exclusivamente pela aluna de mestrado que desenvolve esse trabalho, devido a necessidade de conhecimento interdisciplinar nas áreas de teste de software, programação concorrente, estatística, além do domínio da ferramenta de teste utilizada.

\subsubsection{Seleção das Variáveis}

\section{- Variáveis Independentes:}

- Critérios de teste estruturais para programas concorrentes com memória compartilhada.

- Programas concorrentes em C/PThreads.

- Defeitos semeados.

- Variáveis Dependentes:

- Casos de teste.

- Quantidade de erros revelados por critério.

- Número de elementos requeridos necessários para satisfazer um critério

- Número de elementos não executáveis encontrados.

- Porcentagem de cobertura atingida para satisfação de um determinado critério.

\subsubsection{Projeto do Experimento}

Neste experimento pode-se distinguir dois tipos de sujeitos: a mestranda, que é responsável pelo planejamento e condução da maior parte do experimento e os alunos de pós-graduação convidados, que são responsáveis por inserir defeitos nos programas selecionados dos benchmarks. Foi solicitado aos participantes que inserissem defeitos relacionados à comunicação ou sincronização podendo estes defeitos serem determinísticos ou não determinísticos.

O experimento foi projetado pela aluna de mestrado que tem o experimento como foco de sua dissertação de mestrado. Em sua atuação como condutora do experimento, cabe a 
ela: selecionar os programas dos benchmarks; distribuir os programas selecionados entre os alunos (sujeitos) para que defeitos sejam inseridos; realizar a análise dos programas com defeitos inseridos; e, obter informações relevantes para o seu estudo a partir desses dados.

A avaliação dos fatores (custo, eficácia e strength) e os possíveis tratamentos (critérios de teste) é realizada por meio do teste dos programas concorrentes com defeitos, por um único sujeito que é a aluna de mestrado que conduz o experimento, ou seja um sujeito executando todos os tratamentos. O design desse experimento pode ser classificado como "mais de um fator e mais de um tratamento".

A ferramenta ValiPThread é utilizada durante a condução do experimento. A condutora do experimento é responsável pela instalação da ferramenta de teste nas máquinas que ambientam o experimento.

\subsubsection{Descrição da Instrumentação}

Tendo em vista que o objetivo do experimento foi a avaliação dos critérios de teste estrutural definidos para programas concorrentes apresentados na Seção 3.2.2. Foi necessário selecionar programas para efetuar os testes utilizando os critérios. Foram selecionados trinta e três programas implementados em $\mathrm{C} /$ Pthreads com erros inseridos. Esses programas provém de benchmarks comumente usados na área de teste de programas concorrentes. Foram selecionados 12 programas do benchmark Inspect (Yang, 2011), do benchmark Helgrind (Valgrind-Developers, 2012) foram selecionados 12 programas e do benchmark Rungta (Rungta e Mercer, 2009), 4 programas. Além disso, foram implementados pelos condutores do experimento 5 programas que implementam soluções à problemas clássicos da programação concorrente.

Alguns dos programas selecionados já possuíam defeitos inseridos, esses defeitos correspondem a erros clássicos encontrados no universo da programação concorrente e por isso são considerados no estudo, durante a avaliação dos critérios. Porém o estudo contou também com erros inseridos por alunos de Pós-Graduação. Coube a condutora do experimento retirar os defeitos presentes nos programas selecionados e atribuir de maneira aleatória aos alunos um conjunto de programas nos quais eles eram responsáveis por inserir defeitos. Os programas com defeitos inseridos foram reportados à condutora do experimento para prosseguir o experimento.

Após receber os programas com defeitos inseridos a condutora do experimento fez uma análise, identificando e classificando esses defeitos.

Inicia-se a atividade de teste a fim de avaliar os fatores custo, eficácia e strength dos critérios de teste. A ferramenta de teste ValiPThread foi usada para auxiliar a atividade de 
teste, sua utilização foi essencial para diminuir o esforço e o tempo gasto com a atividade de teste, auxiliando na tarefa de aplicação dos critérios de teste.

A avaliação de cada fator de interesse é feita por meio de um conjunto de casos de teste adequado. Para obter esse conjunto a condutora do experimento deve, para cada um dos programas selecionados e com defeitos inseridos, gerar os elementos requeridos para cada critério de teste, isso é feito de maneira automatizada, utilizando a ferramenta de teste. Em posse dos elementos requeridos, a condutora do experimento desenvolve um conjunto inicial de casos de teste e aplica ao programa obtendo uma cobertura inicial, a partir dai são gerados novos casos de teste de teste para cobrir os elementos remanescentes requeridos pelo critério, até atingir a cobertura total do critério. Durante este passo são determinados os elementos não-executáveis e obtida a cobertura final usando o conjunto total de casos de teste.

A análise dos fatores segue a metodologia proposta na Seção 4.2. A partir desta análise e da aplicação de testes estatísticos conclusões a respeito do objeto de estudo são obtidas.

\subsubsection{Ameaças a Validade do Experimento}

\section{- Validade da conclusão}

A validade da conclusão, corresponde ao fato de serem obtidas conclusões corretas sobre a teoria analisada. As conclusões obtidas como resultado deste experimento podem ser influenciadas pelo teste estatístico escolhido. Para este estudo foram escolhidos testes estatísticos de acordo com a análise de normalidade dos dados e as características dos dados coletados, foi considerada também a relação entre as amostras. Cada amostra corresponde a um fator (critério de teste) com dez tratamentos distintos (all-nodes, all-p-nodes, all-w-nodes, all-edges, all-s-edges, all-c-uses, all-p-uses, all-sync-uses, all-comm-c-uses, all-comm-p-uses). A escolha do teste estatístico apropriado para a variável em estudo foi realizado com base nos fundamentos da estatística experimental. Além disso, o estudo contou com o auxílio de especialistas na área durante a interpretação dos dados resultantes. Com isso, acredita-se ter reduzido uma possível ameaça à validade do estudo.

\section{- Validade interna}

A validade interna está relacionada às conclusões sobre as causas e seus efeitos no estudo. Neste sentido, as diferenças entre os fatores analisados (custo, eficácia e strenth) podem não ser resultantes do tipo do critério utilizado e sim atribuídas a outras causas, como a validade dos elementos requeridos gerados pela ferramenta de teste. Porém neste caso, acreditamos que a implementação da metodologia na ferramenta foi feita corretamente e esta não é considerada uma ameaça à validade. 
Outro fator que pode ser relevante aos efeitos observados, são os defeitos inseridos nos programas, que poderiam influenciar nos resultados observados para eficácia e strength. Entretanto, os defeitos inseridos foram classificados sob uma taxonomia, o que pode assegurar que esses defeitos são abrangentes de maneira geral, pois envolvem diversas categorias da classificação, mitigando o viés, de que eles pudessem influenciar negativamente nas análises.

\section{- Validade de construção}

A validade de construção está relacionada ao fato do experimento refletir de maneira realística a influência das causas (tratamentos) nos efeitos (resultados observados). Nesse estudo, esta ameaça está relacionada com o conhecimento do pesquisador sobre os programas usados e falhas inseridas, que pode influenciar a aplicação dos critérios de teste. Isso poderia ser uma ameaça ao estudo se o condutor realizasse o experimento pensando nas hipóteses definidas e nos resultados que esperava obter. Para minimizar esse viés, para cada um dos programas avaliados os critérios utilizados eram selecionados um a um de maneira aleatória. Prevenindo que o conhecimento sobre os programas e os defeitos inseridos influenciassem a geração de casos de teste. Além disso, optou-se pelo uso de defeitos inseridos por outros sujeitos, evitando viés conforme conhecimento dos critérios de teste.

\section{- Validade externa}

A validade externa refere-se à habilidade de generalizar os resultados do estudo experimental para outros contextos como, por exemplo, considerando outra população no estudo. Esse estudo foi desenvolvido de modo a possibilitar sua replicação para comparar novas e diferentes abordagens de teste para programas concorrentes, entretanto, a generalização dos resultados para outras populações pode não ser possível. A instrumentação utilizada na condução deste experimento talvez não seja adequada à utilização em maior abrangência na academia, pois apesar dos programas refletirem práticas comuns na programação concorrente, não é possível garantir que todos os casos foram cobertos. Os programas escolhidos procuram considerar diferentes características da programação concorrente, tentando contemplar o que é encontrado em programas reais.

\subsection{Operação}

A operação do experimento consiste na preparação dos sujeitos, do material utilizado e do ambiente em que o experimento será conduzido; na execução do experimento de acordo como o planejamento definido e a validação dos dados coletados. 


\subsubsection{Preparação}

Antes do início da execução do experimento é necessário que o ambiente para os testes tenha sido preparado. Devido aos testes serem realizados com o auxilio da ferramenta de teste ValiPThread, durante a preparação do experimento ela deve ser instalada e configurada. A ferramenta exige uma configuração especial do ambiente para sua instalação e funcionamento adequado, que compreende:

- Sistema Operacional Ubuntu 8.04;

- GNU bash, versão 3.2.39;

- Ferramenta IDeL instanciada para linguagem C;

- e o pacote libstdc++2.10-glibc2.2, requerido pela ferramenta IDeL.

Com o ambiente de teste pronto, é necessário selecionar os programas que serão usados para realização dos testes. Foram selecionados 33 programas em $C /$ Pthread provenientes de benchmarks de teste. Após selecionados os programas, cabe a condutora do experimento distribuir os programas entre os sujeitos participantes do experimento, para que defeitos sejam inseridos. Alguns dos programas provenientes dos benchmarks já apresentavam defeitos inseridos e por esse motivo esses defeitos foram retirados antes do envio aos sujeitos. Porém, durante os testes esses defeitos são novamente considerados.

De posse dos programas já com defeitos inseridos e instalação da ferramenta de teste, o ambiente do experimento já está pronto podendo seguir para a próxima fase que é a coleta de dados por meio da execução do experimento. Maiores detalhes a respeito da instalação e utilização da ferramenta ValiPThread podem obtidos nos trabalhos de (Sarmanho, 2009; Sarmanho et al., 2008).

\subsubsection{Execução}

O experimento foi realizado em 3 etapas. A primeira etapa compreendeu a seleção dos programas que fariam parte do experimento a partir de um estudo sobre benchmarks disponíveis na literatura para avaliação de técnicas e métodos de teste de programas concorrentes com memória compartilhada. A partir deste estudo, três benchmarks comumente usados para avaliação de técnicas e critérios de testes no contexto de programas concorrentes foram selecionados: Inspect (Yang, 2011) (12 programas), Helgrind (Valgrind-Developers, 2012) (12 programas) e Rungta (Rungta e Mercer, 2009) (4 programas). Além dos programas dos benchmarks foram selecionados cinco programas que implementam soluções para problemas clássicos da programação concorrente. Na Tabela 
3.1 são apresentados os 33 programas utilizados no experimento e algumas informações relacionadas a sua complexidade como o número de linhas de código de cada programa (LOC), o número de threads e o número de operações post e wait.

Tabela 3.1: Programas selecionados.

\begin{tabular}{|c|c|c|c|c|c|}
\hline & Programas & $\mathrm{LOC}$ & Threads & Posts & Waits \\
\hline \multirow{5}{*}{ 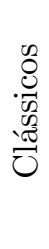 } & Sleeping barber & 62 & 4 & 4 & 4 \\
\hline & GCD & 101 & 4 & 11 & 11 \\
\hline & Jacobi & 192 & 5 & 12 & 9 \\
\hline & MMult & 148 & 13 & 4 & 4 \\
\hline & ProdCons & 97 & 5 & 5 & 4 \\
\hline \multirow{12}{*}{$\begin{array}{l}\stackrel{+}{0} \\
\stackrel{0}{0} \\
\tilde{\Xi} \\
\Xi\end{array}$} & Carter01 & 45 & 3 & 6 & 6 \\
\hline & Deadlock01 & 30 & 3 & 4 & 4 \\
\hline & Dpor-example1 & 73 & 4 & 3 & 3 \\
\hline & Dpor-example2 & 104 & 4 & 9 & 6 \\
\hline & Dpor-example3 & 30 & 4 & 0 & 0 \\
\hline & Lazy01 & 39 & 4 & 3 & 3 \\
\hline & Phase01 & 25 & 3 & 4 & 4 \\
\hline & Race01 & 15 & 3 & 0 & 0 \\
\hline & Rafkind01 & 15 & 2 & 1 & 1 \\
\hline & Simple1 & 27 & 3 & 2 & 2 \\
\hline & Stateful01 & 34 & 3 & 4 & 4 \\
\hline & Stateful06 & 40 & 3 & 2 & 2 \\
\hline \multirow{12}{*}{ 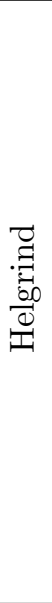 } & Hg01_all_ok & 21 & 3 & 2 & 2 \\
\hline & Hg02_deadlock & 30 & 3 & 5 & 5 \\
\hline & Hg03_inherit & 41 & 3 & 0 & 0 \\
\hline & Hg04_race & 16 & 3 & 0 & 0 \\
\hline & Hg06_readshared & 21 & 3 & 0 & 0 \\
\hline & Tc01_simple_race & 19 & 2 & 0 & 0 \\
\hline & Tc02_simple_tls & 20 & 2 & 0 & 0 \\
\hline & Tc05_simple_race & 28 & 2 & 2 & 2 \\
\hline & Tc06_two_races & 30 & 2 & 2 & 0 \\
\hline & Tc13_laog & 32 & 1 & 4 & 4 \\
\hline & Tc16_byterace & 25 & 1 & 0 & 0 \\
\hline & Tc18_semabuse & 26 & 1 & 1 & 1 \\
\hline \multirow{4}{*}{ 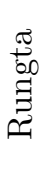 } & Account & 91 & 3 & 6 & 6 \\
\hline & Reorder & 66 & 5 & 0 & 0 \\
\hline & Twostage & 82 & 3 & 5 & 4 \\
\hline & Wronglock & 67 & 3 & 5 & 4 \\
\hline
\end{tabular}

A próxima fase da execução do experimento compreende a atribuição aleatória dos programas selecionados aos alunos de pós-graduação para a inserção de defeitos nos programas. A maioria dos programas do benchmark já continham algum tipo de defeito, os quais foram considerados no estudo. Para que os participantes inserissem novos defeitos, versões corretas desses programas foram geradas e disponibilizadas aos partici- 
pantes. Após a inserção de defeitos, a condutora do experimento classificou os defeitos seguindo a taxonomia de defeitos proposta por Bradbury (Bradbury e Jalbert, 2009) que traz uma classificação para defeitos típicos cometidos por programadores que utilizam o padrão PThreads. A Tabela 3.2 sumariza a quantidade de defeitos inseridos para cada uma das categorias distintas de defeitos propostos na taxonomia. Cada defeito deu origem a um programa que foi executado na ferramenta ValiPThread. Optou-se por adicionar somente um defeito por programa, procurando evitar que os defeitos se combinassem de alguma forma. A última etapa do processo de execução do experimento envolve apenas a condutora do experimento. Essa etapa corresponde a execução dos testes com o auxílio da ferramenta ValiPThread e também a coleta dos dados gerados. Essa etapa pode ser definida em um conjunto de passos seguindo a seguinte sequência:

1. Um dos 33 programas é selecionado seguindo a ordem crescente de complexidade, ou seja primeiro é selecionado o programa com menor quantidade de linhas de código e instruções de sincronização e comunicação ao final dos testes com este programa outro mais complexo é selecionado e a avaliação continua até a avaliação completa dos 33 programas.

2. O programa selecionado é executado na ferramenta. A cada execução, é selecionado aleatoriamente um critério por vez para geração dos elementos requeridos. Com base nos elementos requeridos, a aluna de mestrado é responsável por gerar casos de teste que cubram esses elementos.

3. A coleta dos dados é feita por meio de planilhas eletrônicas e durante a execução dos testes, o testador deve informar cada novo caso de teste inserido para cada nova entrada apenas um identificador deve estar associado. A cada caso de teste executado, os elementos requeridos cobertos devem ser informados e a taxa de cobertura parcial respectivamente.

4. Ao final dos testes (o fim dos testes é determinado pelo alcance da máxima cobertura para todos os critérios avaliados), três planilhas com os dados devem ser produzidas. A primeira planilha contêm informações a respeito do número total de elementos requeridos gerados pela ferramenta, o número total de elementos cobertos pelos casos de teste gerados e uma relação dos elementos não-executáveis identificados pelo testador. Essas informações serão utilizadas durante a análise dos dados a fim de obter conclusões a respeito do custo de aplicação dos critérios. A segunda planilha deve conter informações sobre a eficácia dos critérios em revelar defeitos, para isso ao final dos testes é verificado se o defeito inserido no programa foi revelado com a cobertura de cada um dos critérios de teste avaliados cada defeito identificado é então classificado de acordo com a taxonomia utilizada. A última planilha contém as informações sobre a dificuldade de satisfação dos critérios, o conjunto adequado a um critério é aplicado a todos os demais critérios e a 
porcentagem de cobertura atingida é armazenada na planilha para que posteriormente seja realizada a análise dos dados e testes estatísticos.

Tabela 3.2: Classificação dos erros inseridos.

\begin{tabular}{|l|c|}
\hline Tipos de defeito & Inseridos \\
\hline 1. Deadlock & 70 \\
\hline 2. Bloqueio errado ou falta de bloqueio & 61 \\
\hline 3. Interferência & 80 \\
\hline 4. Inicialização de contagem incorreta & 30 \\
\hline 5. Exaustão de recursos & 13 \\
\hline 6. Bloqueio na região crítica & 36 \\
\hline 7. Operações não atômicas assumidas como atômicas & 4 \\
\hline 8. Sleep & 15 \\
\hline 9. Perdendo sinais ou sinais inexistentes & 16 \\
\hline 10. Perdendo uma notificação & 13 \\
\hline
\end{tabular}

5. Os passos 3 e 4 devem ser repetidos para um mesmo programa, considerando cada um dos critérios de teste selecionados para este experimento.

6. Ao final da execução do teste para todos os programas e todos os critérios de teste, as planilhas devem ser analisadas para obtenção das informações e análise dos dados.

7. Todos os artefatos utilizados no experimento são reunidos em um pacote de laboratório. Esse pacote tem acesso público e pode ser usado na replicação do experimento em outros ambientes e na comparação com outras teorias.

Os dados coletados na fase de execução do experimento são inspecionados a fim de encontrar qualquer dado inválido, ao final da inspeção os dados são validados pelos pesquisadores envolvidos no projeto.

\subsection{Considerações Finais}

Este capítulo abordou de maneira padronizada a definição do experimento com base na análise do objetivo principal do estudo. Expôs também algumas possíveis ameaças a sua validade dadas as dificuldades inerentes a este estudo. Abordou de maneira detalhada o processo de operação do experimento envolvendo sua execução e coleta de dados. Listou os requisitos exigidos do ambiente para execução controlada do experimento. Foram apresentados de maneira resumida os dados coletados durante a execução do experimento para cada um dos fatores avaliados, cuja análise é abordada no próximo capítulo. 


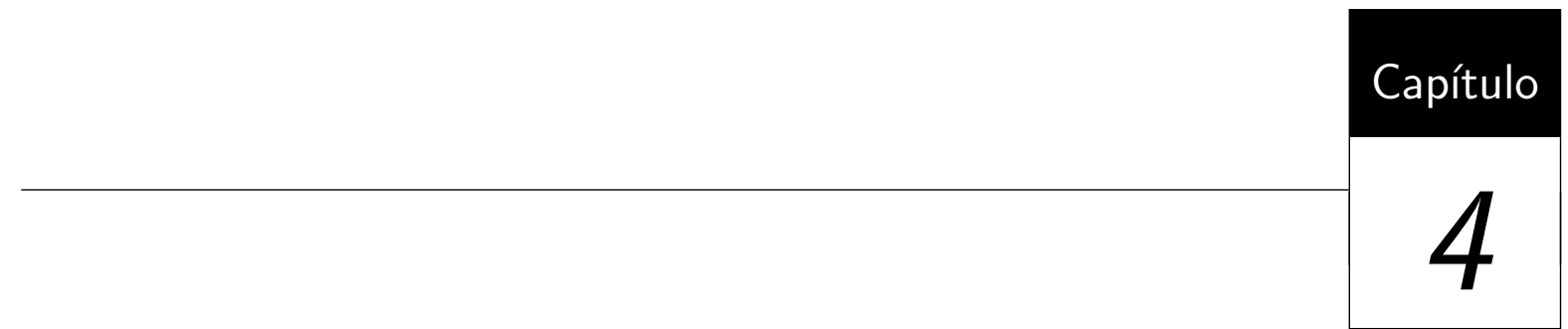

\section{Análise dos Resultados}

\subsection{Considerações Iniciais}

Neste capítulo, os dados coletados durante a execução do experimento são analisados com base nos princípios da estatística descritiva. O teste de hipóteses é realizado a fim de validar a pesquisa e obter conclusões a respeito dos fatores avaliados no estudo. As principais conclusões são então sumarizadas e apresentadas.

A Seção 4.2 apresenta a análise dos dados por meio da estatística descritiva. A Seção 4.3 descreve a eliminação de dados redundantes ou incorretos do estudo. Na Seção 4.4 as hipóteses são testadas com base em métodos estatísticos definidos. Na Seção 4.5 é apresentada uma comparação entre os critérios sequenciais e os concorrentes. Na Seção 4.6 é descrito o modo como os resultados do estudo são apresentados e empacotados. E por fim, na Seção 4.7 são apresentadas algumas considerações sobre os resultados obtidos.

\subsection{Estatística Descritiva}

No processo de análise dos resultados, os dados coletados durante a execução do experimento são avaliados com base nos princípios da estatística descritiva. A estatística descritiva é utilizada para organizar e sumarizar os dados de maneira que possam ser melhor compreendidos, porém não possibilita inferir algo ou tirar conclusões sobre a po- 
pulação. O teste de hipóteses é realizado a fim de validar a pesquisa e obter conclusões a respeito do objeto de estudo.

\subsubsection{Análise do Custo}

Em relação ao custo, três aspectos foram analisados: quantidade de casos de teste necessários para satisfazer cada critério, quantidade de elementos requeridos e quantidade de elementos não-executáveis.

A Tabela 4.1 apresenta os valores de custo relacionado ao tamanho do conjunto de casos de teste adequado (considerando somente casos de teste efetivos) para cada um dos critérios de teste avaliados em relação a cada um dos 33 programas utilizados no experimento. A primeira coluna da tabela lista os programas dos benchmarks utilizados no experimento, da segunda coluna até a décima primeira coluna são discriminados o número de casos de testes que compõe o conjunto adequado para cada um dos critérios avaliados, all-nodes (AN), all-p-nodes (ANP), all-w-nodes (ANW), all-edges (AE), all-s-edges (AES), all-c-uses (ACU), all-p-uses (APU), all-sync-uses (ASU), all-comm-c-uses (ACCU) e all-comm-p-uses (ACPU). Para programas que não geraram elementos requeridos para um dado critério, a célula da tabela correspondente a este programa e critério apresenta o símbolo "*" que representa a ausência de elementos. Programas que requerem valores de entrada, como Mmult, GCD e Jacobi, por exemplo, necessitam de um número maior de casos de teste para cobrir os elementos requeridos gerados, pois cada nova entrada pode levar a execução de caminhos diferentes além dos caminhos correspondentes à execução de novos pares de sincronização. Já os programas que não necessitam valores de entrada dependem apenas da execução de pares diferentes de sincronização para a cobertura de novos caminhos e novos elementos requeridos, tornando o conjunto adequado consideravelmente menor para estes programas.

A fim de analisar o comportamento da amostra selecionada para este estudo, os dados foram organizados em gráficos de barras e gráficos boxplot para cada fator analisado. A Figura 4.1 ilustra o custo médio para cada um dos critérios de teste em relação ao tamanho do conjunto de casos de teste adequado para cada benchmark do experimento.

A análise do histograma sugere a existência de diferenças de custo entre os critérios analisados. Podemos dizer que para essa amostra, os critérios mais custosos em relação à quantidade de casos de teste que compõe o conjunto adequado são os critérios all-edges (AE), all-s-edges (AES), all-sync-uses (ASU) e all-comm-c-uses (ACCU) respectivamente. Já o critério all-p-uses (APU) apresenta variabilidade significativa dos dados o que torna difícil obter conclusões sobre suas propriedades a partir deste gráfico. Os demais critérios apresentam baixo custo se comparado aos anteriores, seguindo uma ordem crescente de 


\section{CAPÍTULO 4. ANÁLISE DOS RESULTADOS}

Tabela 4.1: Custo por critério considerando o conjunto de casos de teste adequado

\begin{tabular}{|c|c|c|c|c|c|c|c|c|c|c|c|}
\hline & Programa & AN & ANP & ANW & $\mathbf{A E}$ & AES & ACU & APU & ASU & $\mathrm{ACCU}$ & ACPU \\
\hline \multirow{5}{*}{$\begin{array}{l}0 \\
0 \\
.0 \\
0 \\
0 \\
0 \\
0 \\
0\end{array}$} & Sleeping barber & 1 & 1 & 1 & 16 & 16 & 1 & 1 & 16 & 1 & 1 \\
\hline & GCD & 8 & 3 & 5 & 17 & 14 & 7 & 8 & 12 & 7 & 2 \\
\hline & Jacobi & 4 & 2 & 3 & 11 & 11 & 2 & 2 & 14 & 6 & 5 \\
\hline & Mmult & 12 & 12 & 12 & 52 & 52 & 12 & 12 & 52 & 24 & $*$ \\
\hline & ProdCons & 2 & 1 & 1 & 6 & 6 & 2 & 1 & 7 & 7 & 2 \\
\hline \multirow{12}{*}{$\begin{array}{l}\vec{U} \\
0 \\
\stackrel{D}{0} \\
\Xi \\
\Xi\end{array}$} & Carter01 & 1 & 1 & 1 & 5 & 5 & 1 & $*$ & 5 & 1 & 1 \\
\hline & Deadlock01 & 1 & 1 & 1 & 2 & 2 & 1 & $*$ & 2 & 2 & $*$ \\
\hline & Dpor-example1 & 1 & 1 & 1 & 2 & 2 & 2 & 2 & 2 & 1 & 2 \\
\hline & Dpor-example2 & 1 & 1 & 1 & 4 & 4 & 1 & 1 & 4 & 1 & 1 \\
\hline & Dpor-example3 & 1 & 1 & 1 & 3 & 3 & 1 & $*$ & 3 & 3 & $*$ \\
\hline & Lazy01 & 1 & 1 & 1 & 4 & 4 & 1 & $*$ & 4 & 2 & 3 \\
\hline & Phase01 & 1 & 1 & 1 & 7 & 7 & 1 & $*$ & 7 & $*$ & $*$ \\
\hline & Race01 & 1 & 1 & 1 & 2 & 2 & 1 & $*$ & 2 & 2 & $*$ \\
\hline & Rafkind01 & 1 & 1 & 1 & 1 & 1 & 1 & $*$ & 1 & $*$ & $*$ \\
\hline & Simple1 & 1 & 1 & 1 & 2 & 2 & 1 & $*$ & 2 & 2 & $*$ \\
\hline & Stateful01 & 1 & 1 & 1 & 5 & 5 & 1 & $*$ & 5 & 2 & $*$ \\
\hline & Stateful06 & 1 & 1 & 1 & 2 & 2 & 1 & 1 & 2 & 1 & $*$ \\
\hline \multirow{12}{*}{ 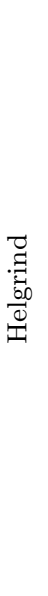 } & Hg01_all_ok & 1 & 1 & 1 & 2 & 2 & 1 & $*$ & 2 & 2 & $*$ \\
\hline & Hg02_deadlock & 1 & 1 & 1 & 2 & 2 & 1 & $*$ & 2 & $*$ & $*$ \\
\hline & Hg03_inherit & 1 & 1 & 1 & 2 & 2 & 1 & $*$ & 2 & $*$ & $*$ \\
\hline & Hg04_race & 1 & 1 & 1 & 2 & 2 & 1 & $*$ & 2 & 2 & $*$ \\
\hline & Hg06_readshared & 1 & 1 & 1 & 1 & 1 & 1 & $*$ & $*$ & * & $*$ \\
\hline & Tc01_simple_race & 1 & 1 & 1 & 2 & 2 & $*$ & 1 & 2 & 2 & $*$ \\
\hline & Tc02_simple_tls & 1 & 1 & 1 & 1 & 1 & $*$ & 1 & 1 & 1 & $*$ \\
\hline & Tc05_simple_race & 1 & 1 & 1 & 2 & 2 & $*$ & 1 & 2 & 2 & 2 \\
\hline & Tc06_two_races & 1 & 1 & 1 & 2 & 2 & $*$ & 1 & 2 & 2 & 2 \\
\hline & Tc13_laog1 & 1 & $*$ & $*$ & 1 & $*$ & 1 & $*$ & $*$ & $*$ & $*$ \\
\hline & Tc16_byterace & 1 & 1 & 1 & 1 & 1 & 1 & 1 & $*$ & $*$ & $*$ \\
\hline & Tc18_semabuse & 1 & 1 & 1 & 1 & 1 & $*$ & $*$ & 1 & $*$ & $*$ \\
\hline \multirow{5}{*}{ 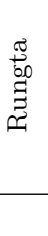 } & Account & 1 & 1 & 1 & 5 & 5 & 1 & 1 & 5 & 1 & 3 \\
\hline & Reorder & 1 & 1 & 1 & 21 & 19 & 1 & 1 & 19 & * & 6 \\
\hline & Twostage & 4 & 3 & 1 & 4 & 2 & 2 & 3 & 4 & 1 & 2 \\
\hline & Wronglock & 3 & 2 & 1 & 4 & 2 & 2 & 3 & 3 & 2 & 1 \\
\hline & Média & 2 & 2 & 2 & 6 & 6 & 2 & 2 & 6 & 3 & 2 \\
\hline
\end{tabular}

custo, podemos classificá-los como segue: all-comm-p-uses (ACPU), all-p-nodes (ANP), all-w-nodes (ANW), all-c-uses (ACU) e all-nodes (AN).

Além do histograma, a análise estatística dos dados é feita utilizando gráficos boxplot 4.2. O gráfico boxplot ilustra o valor mínimo, máximo e a média da amostra. O boxplot é um gráfico definido com base em cinco valores: o valor mínimo, primeiro quartil, mediana, terceiro quartil e valor máximo. Esses cinco valores são calculados ordenando os elementos da amostra, a mediana corresponde ao valor central da amostra, a mediana da metade 


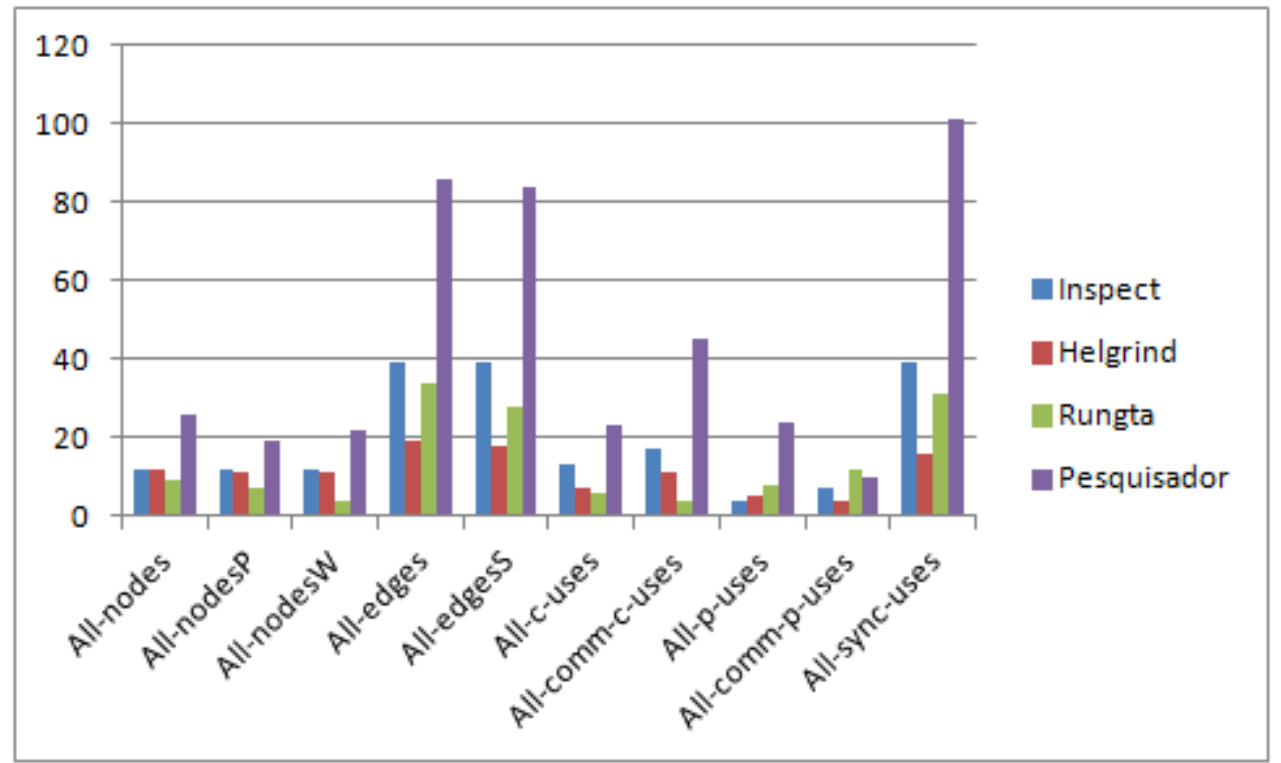

Figura 4.1: Custo relacionado ao tamanho do conjunto de casos de teste adequado.

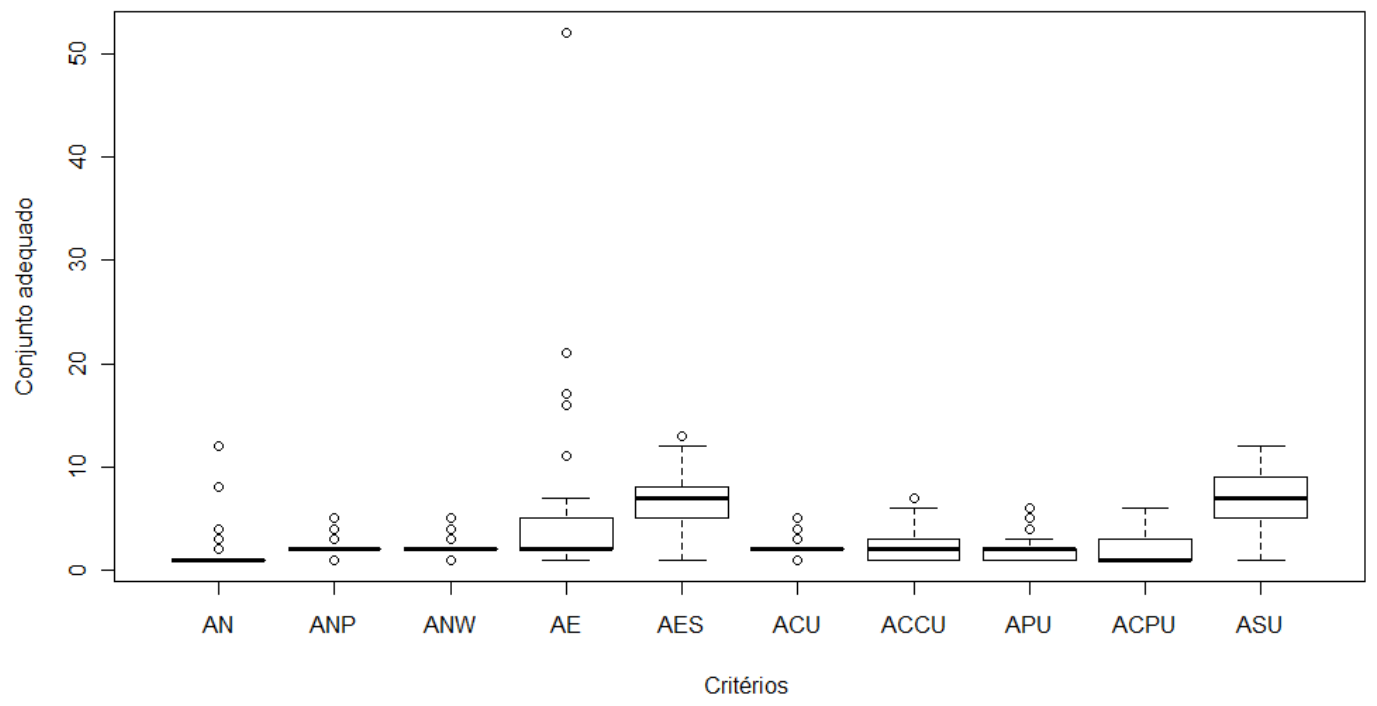

Figura 4.2: Custo dos critérios considerando o conjunto adequado.

dos valores abaixo do valor central corresponde ao primeiro quartil e a mediana dos valores abaixo do valor geral corresponde ao terceiro quartil. Os valores mínimo e máximo correspondem aos valores mínimos e máximos para a amostra respectivamente. O gráfico é composto por uma caixa paralela ao eixo das escalas dos dados, tendo seu início no primeiro quartil e se estendendo até o terceiro quartil. Dentro da caixa há uma linha que corresponde ao valor da mediana. Há também linhas paralelas à escala que vão das extremidades da caixa até o valor extremo (máximo e mínimo) dos dados, essas linhas são 
comumente chamadas de bigodes. Além dessas informações, são representados em forma de pontos dispersos os valores atípicos para a amostra, denominados outliers.

Considerando ainda a análise de custo em relação ao conjunto de casos de teste adequados, o boxplot apresentado na Figura 4.2 ilustra a variabilidade dos dados da amostra para cada um dos critérios. Com base na análise deste gráfico podemos inferir que os critérios all-edges (AE), all-s-edges (AES), all-p-uses (APU), all-comm-c-uses (ACCU) e all-comm-p-uses (ACPU) tendem a apresentar valores mais elevados para o fator custo e uma maior dispersão dos dados, pois os valores médios "linha no meio da caixa" para esses critérios são maiores e o tamanho da caixa que representa a variabilidade dos dados também é maior. Além disso, podemos identificar alguns outliers "pontos fora das caixas" que constituem valores que dispersam dos demais para a amostra e devem ser investigados na próxima etapa da análise de dados.

A Tabela 4.2 apresenta a análise do custo em relação a elementos requeridos. Assim, para cada um dos programas que compõe o experimento, a tabela apresenta a quantidade de elementos requeridos para cada critério, pelo número de elementos não executáveis identificados pelo testador.

Os resultados do custo em relação ao número de elementos requeridos mostram que, assim como para o conjunto adequado, os programas com entradas distintas requerem um maior número de elementos requeridos. Em relação à quantidade de elementos não-executáveis identificados, pode-se concluir que, de maneira geral, critérios que apresentam muitos elementos requeridos consequentemente apresentam um maior número de elementos não-executáveis, como é o caso dos critérios all-edges (AE), all-s-edges (AES) e all-sync-uses (ASU). Porém, os critérios all-comm-p-use (ACPU) e all-comm-c-use (ACCU), que apresentam um conjunto de elementos requeridos reduzido para a maioria dos programas, apresentam uma quantidade elevada de elementos não-executáveis. Esse fato pode decorrer do modo como são definidos os elementos requeridos, os quais são gerados de maneira conservadora, considerando todos os pares possíveis de definição e uso sem considerar elementos não executáveis. Isso ocorre porque não é possível obter de maneira estática os pares definição e uso (isso é obtido somente em tempo de execução, com uma análise pós-mortem).

A Figura 4.3 ilustra os valores médios de custo para cada critério em relação ao número de elementos para cada benchmark analisado. Os resultados são similares aos obtidos considerando o conjunto de casos de teste adequados, porém alguns critérios apresentam-se mais custosos considerando o número de elementos requeridos, como é o caso do critério all-nodes $(\mathrm{AN})$, que apresenta valores de custo mais elevados que os demais critérios, ficando atrás apenas do critério all-edges (AE), ao contrário do que acontecia quando considerado o tamanho do conjunto de casos de teste adequado. 
CAPÍTULO 4. ANÁLISE DOS RESULTADOS

Tabela 4.2: Custo: Elementos requeridos/Elementos não executáveis.

\begin{tabular}{|c|c|c|c|c|c|c|c|c|c|c|}
\hline Programa & $\mathbf{A N}$ & ANP & ANW & $\mathbf{A E}$ & AES & ACU & APU & ASU & ACCU & ACPU \\
\hline Sleeping barber & $82 / 6$ & $29 / 5$ & $19 / 0$ & $76 / 14$ & $67 / 14$ & $27 / 0$ & $8 / 2$ & $57 / 13$ & $2 / 0$ & $4 / 1$ \\
\hline GCD & $161 / 6$ & $27 / 4$ & $25 / 0$ & $130 / 22$ & $81 / 18$ & $44 / 7$ & $118 / 15$ & $60 / 14$ & $39 / 10$ & $8 / 4$ \\
\hline Jacobi & $283 / 27$ & $40 / 9$ & $27 / 2$ & $273 / 130$ & $189 / 118$ & $68 / 1$ & $78 / 16$ & $243 / 158$ & $349 / 256$ & $308 / 247$ \\
\hline Mmult & $353 / 13$ & $66 / 41$ & $49 / 0$ & $539 / 236$ & $444 / 236$ & $247 / 0$ & $236 / 27$ & $404 / 228$ & $448 / 128$ & $*$ \\
\hline ProdCons & $41 / 0$ & $11 / 0$ & $8 / 0$ & $21 / 5$ & $16 / 5$ & $8 / 0$ & $8 / 2$ & $10 / 1$ & $13 / 3$ & $*$ \\
\hline Carter01 & $44 / 3$ & $13 / 0$ & $10 / 0$ & $42 / 19$ & $32 / 14$ & $2 / 0$ & $*$ & $26 / 10$ & $18 / 14$ & $36 / 30$ \\
\hline Deadlock01 & $24 / 0$ & $10 / 0$ & $8 / 0$ & $21 / 6$ & $18 / 6$ & $2 / 0$ & * & $12 / 4$ & $4 / 2$ & $*$ \\
\hline Dpor-example1 & $69 / 4$ & $8 / 2$ & $5 / 0$ & $26 / 6$ & $12 / 4$ & $36 / 2$ & $32 / 9$ & $12 / 4$ & $2 / 0$ & $8 / 4$ \\
\hline Dpor-example2 & $66 / 9$ & $15 / 4$ & $7 / 0$ & $37 / 16$ & $24 / 10$ & $36 / 8$ & $28 / 12$ & $22 / 10$ & $2 / 2$ & $20 / 0$ \\
\hline Dpor-example3 & $30 / 0$ & $12 / 0$ & $10 / 0$ & $28 / 10$ & $24 / 10$ & $3 / 0$ & $*$ & $12 / 4$ & $6 / 3$ & $*$ \\
\hline Lazy01 & $27 / 1$ & $11 / 0$ & $9 / 0$ & $28 / 9$ & $24 / 9$ & $3 / 0$ & $*$ & $12 / 3$ & $3 / 1$ & $3 / 0$ \\
\hline Phase01 & $30 / 0$ & $14 / 0$ & $12 / 0$ & $51 / 22$ & $48 / 22$ & $2 / 0$ & $*$ & $42 / 17$ & $*$ & $*$ \\
\hline Race01 & $18 / 0$ & $7 / 0$ & $6 / 0$ & $17 / 4$ & $14 / 4$ & $2 / 0$ & * & $8 / 3$ & $4 / 2$ & $*$ \\
\hline Rafkind01 & $11 / 0$ & $4 / 4$ & $3 / 3$ & $6 / 1$ & $4 / 1$ & $1 / 0$ & $*$ & $2 / 1$ & $*$ & $*$ \\
\hline Simple1 & $19 / 0$ & $7 / 0$ & $6 / 0$ & $15 / 4$ & $12 / 4$ & $2 / 0$ & $*$ & $6 / 2$ & $4 / 2$ & $*$ \\
\hline Stateful01 & $28 / 0$ & $10 / 0$ & $8 / 0$ & $29 / 12$ & $26 / 12$ & $2 / 0$ & $*$ & $20 / 8$ & $6 / 2$ & $*$ \\
\hline Stateful06 & $26 / 0$ & $7 / 0$ & $6 / 0$ & $17 / 4$ & $12 / 4$ & $8 / 0$ & $8 / 2$ & $6 / 0$ & $3 / 2$ & $*$ \\
\hline Hg01_all_ok & $20 / 0$ & $8 / 0$ & $7 / 0$ & $23 / 9$ & $20 / 9$ & $2 / 0$ & $*$ & $14 / 6$ & $4 / 2$ & * \\
\hline Hg02_deadlock & $24 / 0$ & $11 / 0$ & $9 / 0$ & $27 / 11$ & $24 / 11$ & $2 / 0$ & $*$ & $18 / 9$ & * & $*$ \\
\hline Hg03_inherit & $33 / 4$ & $7 / 0$ & $6 / 0$ & $20 / 8$ & $12 / 4$ & $7 / 0$ & * & $6 / 2$ & * & $*$ \\
\hline Hg04_race & $18 / 0$ & $7 / 0$ & $6 / 0$ & $17 / 4$ & $14 / 4$ & $2 / 0$ & $*$ & $8 / 3$ & $4 / 2$ & $*$ \\
\hline Hg06_readshared & $14 / 0$ & $4 / 0$ & $4 / 0$ & $9 / 2$ & $6 / 2$ & $3 / 0$ & $*$ & * & $*$ & * \\
\hline Tc01_simple_race & $16 / 12$ & $6 / 1$ & $3 / 0$ & $10 / 3$ & $7 / 2$ & $*$ & $2 / 1$ & $6 / 2$ & $4 / 2$ & * \\
\hline Tc02_simple_tls & $15 / 2$ & $5 / 1$ & $2 / 0$ & $6 / 2$ & $3 / 1$ & * & $2 / 1$ & $2 / 1$ & $9 / 7$ & $*$ \\
\hline Tc05_simple_race & $27 / 4$ & $9 / 1$ & $5 / 0$ & $18 / 6$ & $13 / 4$ & $*$ & $2 / 1$ & $12 / 4$ & $12 / 8$ & $8 / 4$ \\
\hline Tc06_two_races & $35 / 4$ & $12 / 1$ & $7 / 0$ & $24 / 8$ & $19 / 6$ & * & $2 / 1$ & $18 / 6$ & $18 / 12$ & $12 / 6$ \\
\hline Tc13_laog1 & $26 / 2$ & $2 / 2$ & $*$ & $1 / 0$ & $*$ & $10 / 0$ & * & $*$ & $*$ & $*$ \\
\hline Tc16_byterace & $24 / 0$ & $3 / 0$ & $1 / 0$ & $9 / 1$ & $1 / 0$ & $16 / 0$ & $18 / 5$ & $*$ & $*$ & $*$ \\
\hline Tc18_semabuse & $13 / 1$ & $5 / 1$ & $2 / 0$ & $5 / 2$ & $3 / 2$ & $*$ & $*$ & $2 / 1$ & * & $*$ \\
\hline Account & $57 / 5$ & $11 / 0$ & $7 / 0$ & $27 / 5$ & $18 / 3$ & $26 / 3$ & $18 / 6$ & $12 / 3$ & $15 / 12$ & $18 / 12$ \\
\hline Reorder & $69 / 5$ & $17 / 0$ & $14 / 0$ & $77 / 15$ & $60 / 13$ & $26 / 0$ & $28 / 4$ & $48 / 6$ & $*$ & $48 / 24$ \\
\hline Twostage & $57 / 6$ & $14 / 3$ & $6 / 0$ & $35 / 14$ & $22 / 13$ & $20 / 0$ & $40 / 7$ & $22 / 15$ & $6 / 3$ & $4 / 2$ \\
\hline Wronglock & $46 / 1$ & $9 / 0$ & $6 / 0$ & $23 / 5$ & $12 / 4$ & $20 / 0$ & $38 / 5$ & $8 / 2$ & $9 / 3$ & $6 / 5$ \\
\hline Média & $55 / 4$ & $13 / 3$ & $9 / 0$ & $51 / 19$ & $40 / 18$ & $22 / 1$ & $39 / 7$ & $38 / 18$ & $41 / 21$ & $37 / 26$ \\
\hline
\end{tabular}

O gráfico box-plot da Figura 4.4 ilustra a variabilidade dos dados em relação ao número de elementos requeridos para cada um dos critérios de teste. A figura evidencia também a presença de outliers para os critérios all-nodes (AN), all-p-nodes (ANP) e all-edges (AE), que devem ser analisados antes da realização do teste de hipóteses.

Em relação ao número de elementos não-executáveis, os resultados para o fator custo foram muito semelhantes aos obtidos para o conjunto adequado, conforme apresentado na Figura 4.5. Nessa figura fica claro também a presença de outliers para o fator custo considerando o número do elementos não-executáveis.

A Figura 4.6 possibilita uma comparação entre os valores de custo para os critérios. Pode-se inferir que os critérios não apresentam diferenças significativas de custo, pois além dos valores médios de custo estarem bem próximos, todos os critérios estão dentro do intervalo de confiança uns dos outros.

Os resultados obtidos dão evidências de que a hipótese nula não poderia ser rejeitada. A hipótese nula afirma que os critérios não apresentam diferença significativa de custo. Por meio da análise dos gráficos notamos que os critérios em geral apresentam custo bastante similar. Porém, esses resultados podem ter sido alterados devido à presença marcante 


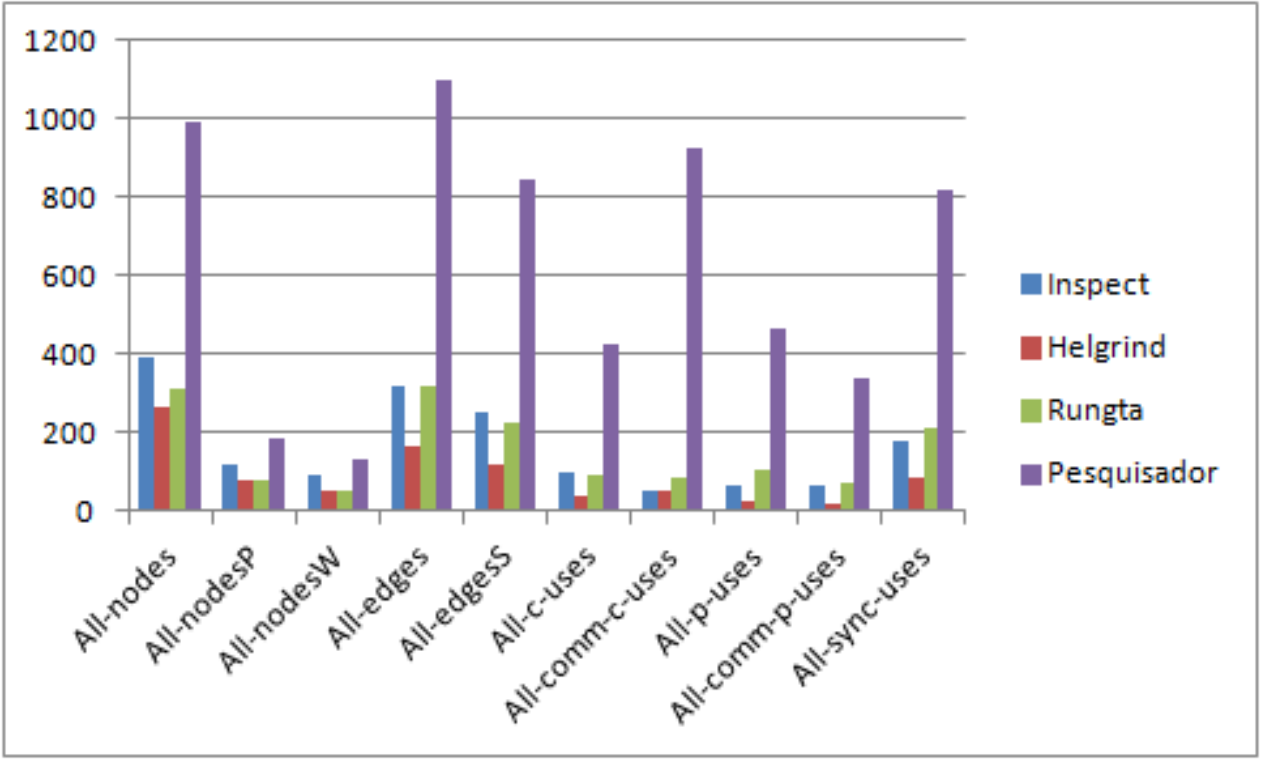

Figura 4.3: Custo relacionado à quantidade de elementos requeridos.

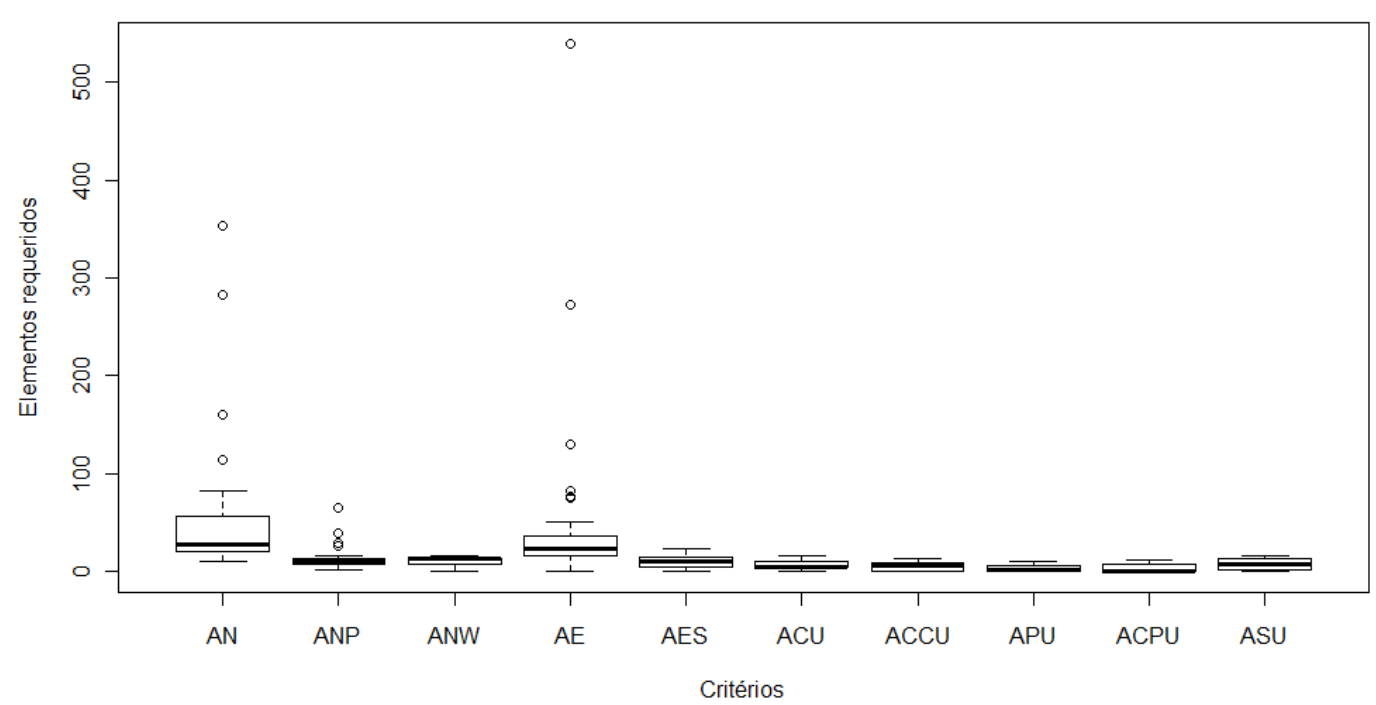

Figura 4.4: Custo dos critérios considerando o número de elementos requeridos.

de diversos outliers na amostra que devem ter sua origem analisada para evitar viés na pesquisa. Desse modo, na Seção 4.4 são apresentados os resultados obtidos por meio do teste estatístico e análise de hipóteses.

\subsubsection{Análise da Eficácia}

Nesse estudo a eficácia está relacionada à quantidade de defeitos que um conjunto de casos de teste adequado a um critério é capaz de revelar. A eficácia é definida, então, pelo 


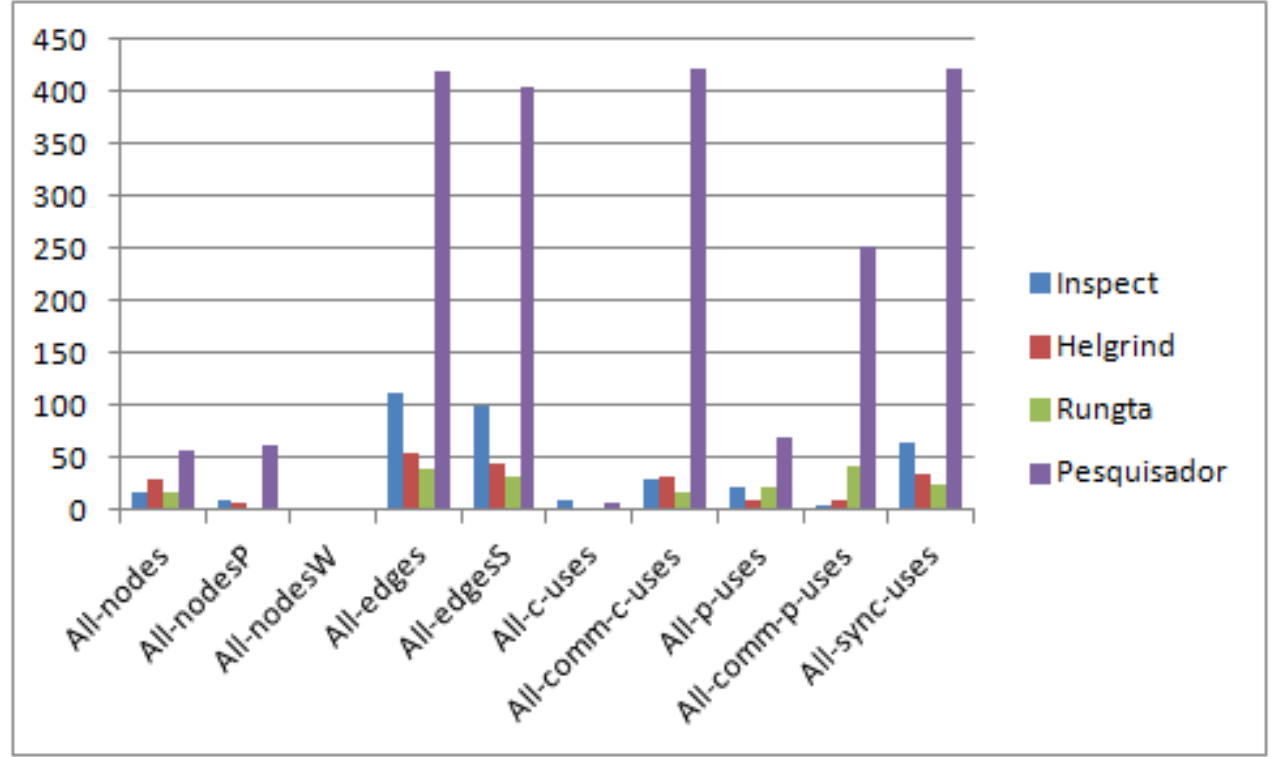

Figura 4.5: Custo relacionado ao número de elementos não executáveis.

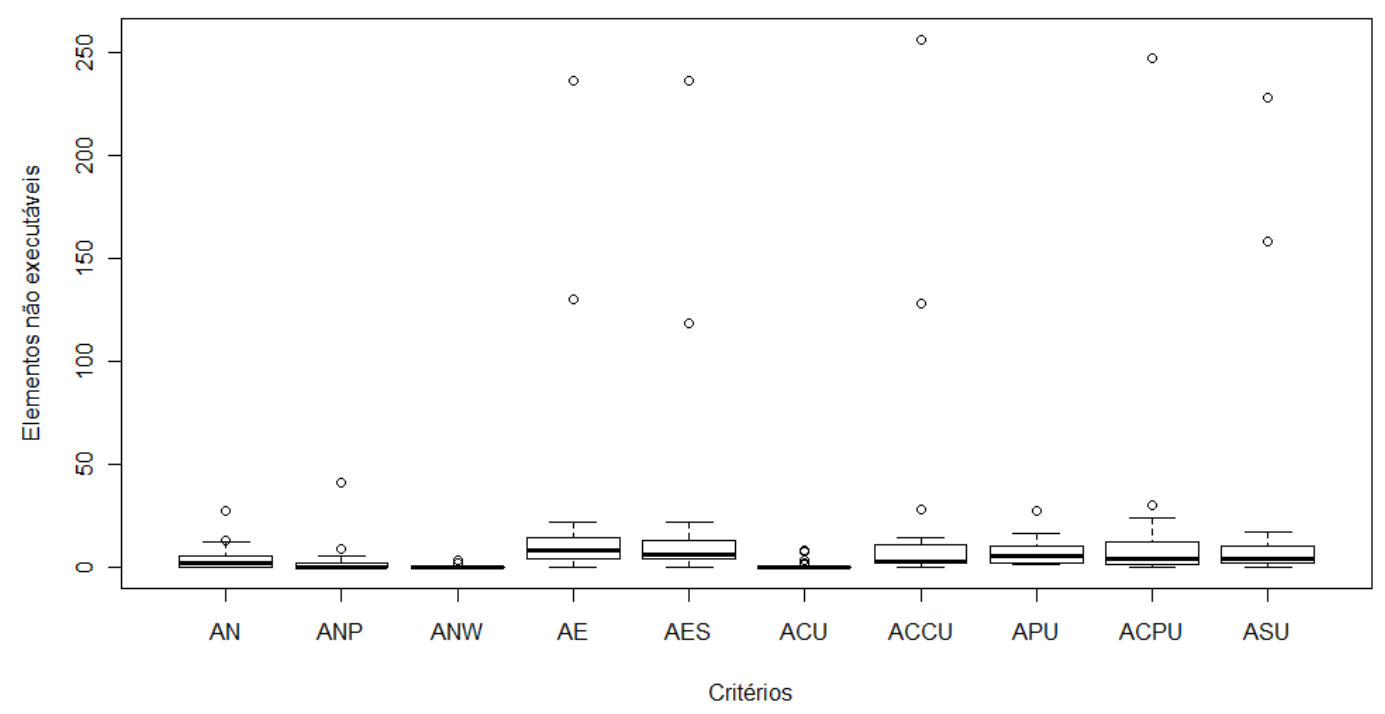

Figura 4.6: Custo dos critérios considerando o número de elementos não executáveis.

cálculo da porcentagem de defeitos revelados pelo critério pelo número total de defeitos inseridos, de acordo com a seguinte fórmula:

$$
\text { Eficacia }=\frac{\text { Numero de defeitos encontrados }}{\text { Numero de defeitos inseridos }} * 100
$$

Para avaliação da eficácia, todas as possíveis sincronizações foram consideradas. Isso é importante porque dependendo da sequência executada um determinado defeito pode não ser revelado. 
CAPÍTULO 4. ANÁLISE DOS RESULTADOS

Tabela 4.3: Eficácia por critério de teste.

\begin{tabular}{|c|c|c|c|c|c|c|c|c|c|c|}
\hline Tipo de defeito & $\mathbf{A N}$ & ANP & ANW & $\mathbf{A E}$ & AES & ACU & APU & ASU & ACCU & ACPU \\
\hline Deadlock & $61 \%$ & $56 \%$ & $54 \%$ & $64 \%$ & $59 \%$ & $53 \%$ & $40 \%$ & $59 \%$ & $57 \%$ & $44 \%$ \\
\hline Bloqueio errado & $75 \%$ & $57 \%$ & $56 \%$ & $82 \%$ & $66 \%$ & $54 \%$ & $51 \%$ & $64 \%$ & $59 \%$ & $52 \%$ \\
\hline Interferência & $31 \%$ & $17 \%$ & $15 \%$ & $35 \%$ & $24 \%$ & $11 \%$ & $17 \%$ & $24 \%$ & $24 \%$ & $26 \%$ \\
\hline Inicialização incorreta & $77 \%$ & $67 \%$ & $63 \%$ & $83 \%$ & $80 \%$ & $63 \%$ & $57 \%$ & $77 \%$ & $63 \%$ & $53 \%$ \\
\hline Exaustão de recursos & $69 \%$ & $61 \%$ & $61 \%$ & $69 \%$ & $61 \%$ & $69 \%$ & $46 \%$ & $61 \%$ & $54 \%$ & $46 \%$ \\
\hline Bloqueio na região crítica & $64 \%$ & $58 \%$ & $58 \%$ & $69 \%$ & $64 \%$ & $56 \%$ & $44 \%$ & $67 \%$ & $56 \%$ & $42 \%$ \\
\hline Atomicidade & $50 \%$ & $0 \%$ & $0 \%$ & $50 \%$ & $0 \%$ & $0 \%$ & $0 \%$ & $0 \%$ & $0 \%$ & $25 \%$ \\
\hline Sleep & $60 \%$ & $47 \%$ & $47 \%$ & $73 \%$ & $60 \%$ & $47 \%$ & $47 \%$ & $60 \%$ & $67 \%$ & $33 \%$ \\
\hline Perda de sinais & $44 \%$ & $44 \%$ & $44 \%$ & $50 \%$ & $50 \%$ & $37 \%$ & $37 \%$ & $50 \%$ & $44 \%$ & $37 \%$ \\
\hline Perda de notificações & $61 \%$ & $61 \%$ & $61 \%$ & $69 \%$ & $69 \%$ & $54 \%$ & $54 \%$ & $69 \%$ & $61 \%$ & $46 \%$ \\
\hline Média & $59 \%$ & $47 \%$ & $46 \%$ & $64 \%$ & $53 \%$ & $44 \%$ & $39 \%$ & $53 \%$ & $49 \%$ & $40 \%$ \\
\hline
\end{tabular}

Na Tabela 4.3 são apresentados os valores relacionados à eficácia para cada um dos critérios avaliados com base na classificação de defeitos apresentada anteriormente na Tabela 3.2. Essa análise considera apenas a quantidade total de defeitos revelados pelo total de defeitos inseridos, não levando em conta se foram ou não gerados elementos para um determinado critério. Com a análise da tabela podemos ranquear os critérios por ordem crescente de eficácia: all-p-uses, all-comm-p-uses, all-c-uses, all-w-nodes, all-p-nodes, all-comm-c-uses, all-sync-uses, all-s-edges, all-nodes e all-eges.

Durante a realização dos testes, foi possível notar que para alguns programas, um determinado tipo de defeito era revelado apenas por algum critério específico. Levando em consideração esse fato, a Tabela 4.4 apresenta a análise de quais critérios, desde que apresentasse elementos requeridos, sempre revelavam um tipo específico de defeito. A análise da tabela mostra que sempre que era possível sua aplicação, os critérios ACCU, ACPU e ASU foram bastante eficazes para revelar defeitos de diferentes categorias. Esses resultados indicam que a aplicação de critérios de teste específicos para programas concorrentes com memória compartilhada, pode contribuir com a atividade de teste, revelando defeitos, que não são descobertos apenas com a aplicação de critérios sequenciais.

Tabela 4.4: Eficácia por tipo de defeito.

\begin{tabular}{|l|l|}
\hline Tipos de erros & Critérios que sempre revelam o defeito \\
\hline 1 - deadlock & ACCU \\
\hline 2 - bloqueio errado & ACPU, ASU \\
\hline 3 - interferência & ACCU,ACPU \\
\hline 4 - inicialização incorreta & AE, AES, ASU, ACCU \\
\hline 5 - exaustão de recursos & AN, ANP, ANW, AE, AES, ACU, APU, ASU, ACCU, ACPU \\
\hline 6 - bloqueio na região crítica & ACCU, ACPU \\
\hline 7 - atomicidade & AN, AE \\
\hline 8 - sleep & AE, ACCU \\
\hline 9 - perdendo sinais & AE, AES, ASU, ACPU \\
\hline 10 - perdendo notificações & $\mathrm{AE}, \mathrm{AES}, \mathrm{ASU}, \mathrm{ACPU}$ \\
\hline
\end{tabular}

A eficácia para os critérios de teste analisados pode ser visualizada na Figura 4.7. Podemos destacar a importância do critério all-comm-p-uses (ACPU) na detecção de defeitos, pois apresentou em média maior eficácia que os demais critérios. Os critérios all-sync-use (ASU), all-s-edges (AES), all-nodes (AN) e all-comm-c-uses (ACCU) também apresentaram boa eficácia ficando a média em torno de 70\%. Os demais critérios apresentaram 
valores para eficácia em torno de $60 \%$ com pouca variabilidade nos dados parecendo não existir diferença significativa de eficácia entre eles.

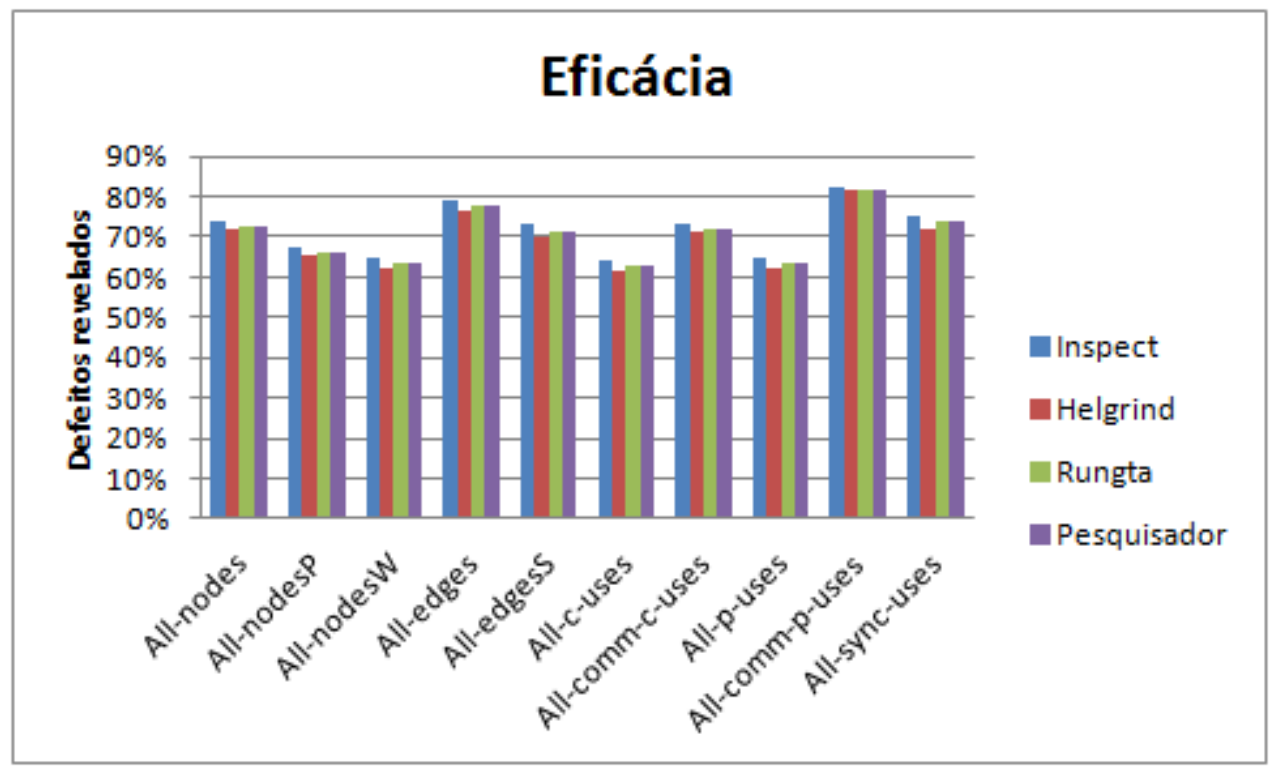

Figura 4.7: Eficácia dos critérios de teste.

O gráfico boxplot apresentado na Figura 4.8 proporciona conclusões similares ao gráfico de barras, porém fica mais clara a variabilidade dos dados. Embora os critérios apresentem, em média, um nível baixo de eficácia para alguns tipos específicos de defeitos, pode ser que para outro tipo apresente maior eficácia. A grande variabilidade dos dados da amostra mostra que conclusões em torno apenas da média não são muito significativas e devem ser melhor investigadas utilizando testes estatísticos.

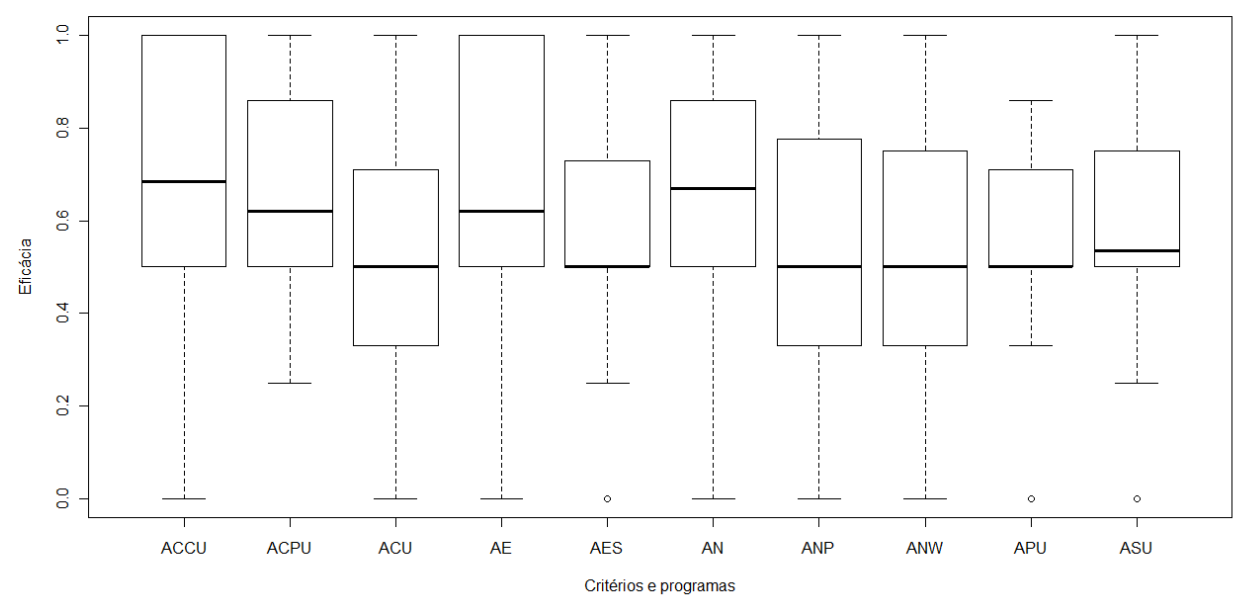

Figura 4.8: Eficácia dos critérios de teste. 


\subsubsection{Análise do Strength}

O strength de um critério está relacionado a possibilidade de um conjunto de casos de teste adequado a um critério $C 2$ também ser adequado a outro critério $C 1$. O objetivo da análise do strength é comparar dois critérios a fim de definir uma estratégia de aplicação de critérios. A métrica utilizada para avaliar o strength de um critério é a cobertura atingida ao aplicar a um critério $C 1$ o conjunto de casos de teste adequado a outro critério $C 2$ $\left(T_{C 2}\right)$. A cobertura alcançada com a aplicação de $T_{C 2}$ em $C 1$ demostra a dificuldade de satisfação de um critério. Uma alta porcentagem de cobertura indica baixo strength e sugere que o critério $C 2$ inclui o critério $C$, então a aplicação do critério $C 1$ depois do critério $C 2$ pode não contribuir para melhorar o conjunto de casos de teste (Wong et al., 1995). A equação a seguir fornece o cálculo do strength para um critério $C 1$.

$$
\text { Strength }_{C_{1}}=\frac{\text { Elementos de } C_{1} \text { cobertos por } T_{C 2}}{\text { Elementos requeridos de } C_{1}-\text { Elementos nao executaveis de } C_{1}} * 100
$$

Para avaliação do strength, os dados foram coletados considerando a aplicação do conjunto de casos de teste adequado a um determinado critério a cada um dos demais critérios analisados. As Tabelas 4.5, 4.6, 4.7, 4.8 e 4.9 apresentam os resultados da aplicação dos conjuntos de casos de teste adequados aos critérios all-nodes, all-p-nodes, all-w-nodes, all-edges e all-s-edges respectivamente, quando aplicados aos demais critérios de teste. As tabelas e análises referentes aos demais critérios são apresentados no Apêndice A.1.

A Figura 4.9 apresenta os dados obtidos considerando o conjunto de casos de teste adequado ao critério all-nodes (AN) aplicado aos demais critérios de teste. Os critérios all-edges (AE), all-s-edges (AES), all-sync-uses (ASU), all-comm-c-uses (ACCU) e allcomm-p-uses (ACPU) apresentam alto strength em relação ao critério all-nodes se considerarmos as médias obtidas para estes critérios, possuindo também grande variabilidade nos dados. Os critérios all-c-uses (ACU) e all-p-uses (APU) apresentaram baixa dificuldade de satisfação em relação ao critério all-nodes, porém embora possuam valores constantes em média, apresentaram alguns outliers para a amostra, o que pode levar a não inclusão destes critérios pelo critério all-nodes. Isso deve ser levado em consideração na realização do teste de hipóteses.

Os dados relacionados ao strength do critério all-p-nodes (ANP) são apresentados na Figura 4.10. Analisando esta figura podemos inferir que o critério all-w-nodes (ANW) apresenta baixo strength para os critérios all-nodes (AN) e all-c-uses (ACU) em média para os valores da amostra, porém outliers são identificados para esses critérios e devem ser analisados. Com alto índice de strength estão os critérios all-edges (AE), all-s-edges (AES), all-p-uses (APU), all-sync-uses (ASU) all-comm-c-uses (ACCU) e all-comm-p-uses (ACPU) e também grande variabilidade nos dados quando comparados ao critério all-p-nodes. 


\section{CAPÍTULO 4. ANÁLISE DOS RESULTADOS}

Tabela 4.5: Aplicação do conjunto de casos de teste adequado ao critério all-nodes (AN) aos demais critérios.

\begin{tabular}{|c|c|c|c|c|c|c|c|c|c|}
\hline Programa & ANP & ANW & $\mathbf{A E}$ & AES & $\mathrm{ACU}$ & APU & ASU & $\mathrm{ACCU}$ & ACPU \\
\hline Sleeping barber & $100 \%$ & $100 \%$ & $100 \%$ & $100 \%$ & $100 \%$ & $*$ & $*$ & $*$ & $*$ \\
\hline GCD & $100 \%$ & $100 \%$ & $94 \%$ & $91 \%$ & $100 \%$ & $100 \%$ & $84 \%$ & $*$ & $*$ \\
\hline Jacobi & $100 \%$ & $100 \%$ & $83 \%$ & $75 \%$ & $*$ & $100 \%$ & $50 \%$ & $75 \%$ & $*$ \\
\hline Mmult & $100 \%$ & $100 \%$ & $93 \%$ & $86 \%$ & $100 \%$ & $100 \%$ & $*$ & $97 \%$ & $89 \%$ \\
\hline ProdCons & $100 \%$ & $100 \%$ & $100 \%$ & $100 \%$ & $06 \%$ & $62 \%$ & $33 \%$ & $100 \%$ & $91 \%$ \\
\hline Carter01 & $100 \%$ & $100 \%$ & $78 \%$ & $64 \%$ & $100 \%$ & $100 \%$ & $63 \%$ & $86 \%$ & $100 \%$ \\
\hline Deadlock01 & $*$ & $100 \%$ & $100 \%$ & $*$ & $100 \%$ & $*$ & $*$ & $*$ & $*$ \\
\hline Dpor-example1 & $100 \%$ & $100 \%$ & $100 \%$ & $100 \%$ & $100 \%$ & * & $*$ & $100 \%$ & $*$ \\
\hline Dpor-example2 & $100 \%$ & $100 \%$ & $100 \%$ & $100 \%$ & $100 \%$ & $100 \%$ & $100 \%$ & $*$ & $*$ \\
\hline Dpor-example3 & $100 \%$ & $100 \%$ & $100 \%$ & $100 \%$ & $*$ & $100 \%$ & $*$ & $100 \%$ & $100 \%$ \\
\hline Lazy01 & $100 \%$ & $100 \%$ & $57 \%$ & $44 \%$ & $100 \%$ & $*$ & $38 \%$ & $100 \%$ & $100 \%$ \\
\hline Phase01 & $100 \%$ & $100 \%$ & $37 \%$ & $57 \%$ & $100 \%$ & $*$ & $36 \%$ & $*$ & $*$ \\
\hline Race01 & $92 \%$ & $100 \%$ & $92 \%$ & $93 \%$ & $100 \%$ & $83 \%$ & $100 \%$ & $100 \%$ & $100 \%$ \\
\hline Rafkind01 & $100 \%$ & $100 \%$ & $82 \%$ & $75 \%$ & $100 \%$ & $*$ & $50 \%$ & $*$ & $*$ \\
\hline Simple1 & $100 \%$ & $100 \%$ & $78 \%$ & $67 \%$ & $100 \%$ & $*$ & $100 \%$ & $100 \%$ & $*$ \\
\hline Stateful01 & $100 \%$ & $100 \%$ & $71 \%$ & $18 \%$ & $100 \%$ & $100 \%$ & $*$ & $*$ & * \\
\hline Stateful06 & $100 \%$ & $100 \%$ & $69 \%$ & $60 \%$ & $100 \%$ & $*$ & $67 \%$ & $50 \%$ & $*$ \\
\hline Hg01_all_ok & $100 \%$ & $100 \%$ & $72 \%$ & $59 \%$ & $100 \%$ & $100 \%$ & $53 \%$ & $78 \%$ & * \\
\hline Hg02_deadlock & $100 \%$ & $100 \%$ & $53 \%$ & $43 \%$ & $100 \%$ & $*$ & $33 \%$ & $50 \%$ & $*$ \\
\hline Hg03_inherit & $100 \%$ & $100 \%$ & $83 \%$ & $77 \%$ & $100 \%$ & $100 \%$ & $67 \%$ & $60 \%$ & $75 \%$ \\
\hline Hg04_race & $100 \%$ & $100 \%$ & $69 \%$ & $67 \%$ & $100 \%$ & $*$ & $50 \%$ & $50 \%$ & $*$ \\
\hline Hg06_readshared & $100 \%$ & $100 \%$ & $100 \%$ & $100 \%$ & $100 \%$ & $100 \%$ & $*$ & $*$ & $*$ \\
\hline Tc01_simple_race & $100 \%$ & $100 \%$ & $100 \%$ & $100 \%$ & $100 \%$ & $100 \%$ & $100 \%$ & $*$ & $*$ \\
\hline Tc02_simple_tls & $100 \%$ & $100 \%$ & $73 \%$ & $67 \%$ & $100 \%$ & $*$ & $56 \%$ & $*$ & $*$ \\
\hline Tc05_simple_race & $100 \%$ & $100 \%$ & $85 \%$ & $75 \%$ & $100 \%$ & $100 \%$ & $67 \%$ & $100 \%$ & $*$ \\
\hline Tc06_two_races & $100 \%$ & $100 \%$ & $100 \%$ & $100 \%$ & $100 \%$ & $*$ & $*$ & $*$ & $*$ \\
\hline Tc13_laog1 & $100 \%$ & $100 \%$ & $100 \%$ & $100 \%$ & $*$ & $*$ & $100 \%$ & $*$ & $*$ \\
\hline Tc16_byterace & $100 \%$ & $100 \%$ & $78 \%$ & $67 \%$ & $100 \%$ & $*$ & $100 \%$ & $100 \%$ & $*$ \\
\hline Tc18_semabuse & $100 \%$ & $100 \%$ & $100 \%$ & $100 \%$ & $*$ & $100 \%$ & $*$ & $100 \%$ & $*$ \\
\hline Account & $100 \%$ & $100 \%$ & $83 \%$ & $75 \%$ & $*$ & $100 \%$ & $50 \%$ & $83 \%$ & $*$ \\
\hline Reorder & $100 \%$ & $100 \%$ & $100 \%$ & $100 \%$ & $100 \%$ & $*$ & $100 \%$ & $100 \%$ & $*$ \\
\hline Twostage & $100 \%$ & $100 \%$ & $100 \%$ & $100 \%$ & $100 \%$ & $100 \%$ & $100 \%$ & $*$ & $100 \%$ \\
\hline Wronglock & $100 \%$ & $100 \%$ & $68 \%$ & $60 \%$ & $100 \%$ & $*$ & $33 \%$ & $50 \%$ & $33 \%$ \\
\hline
\end{tabular}

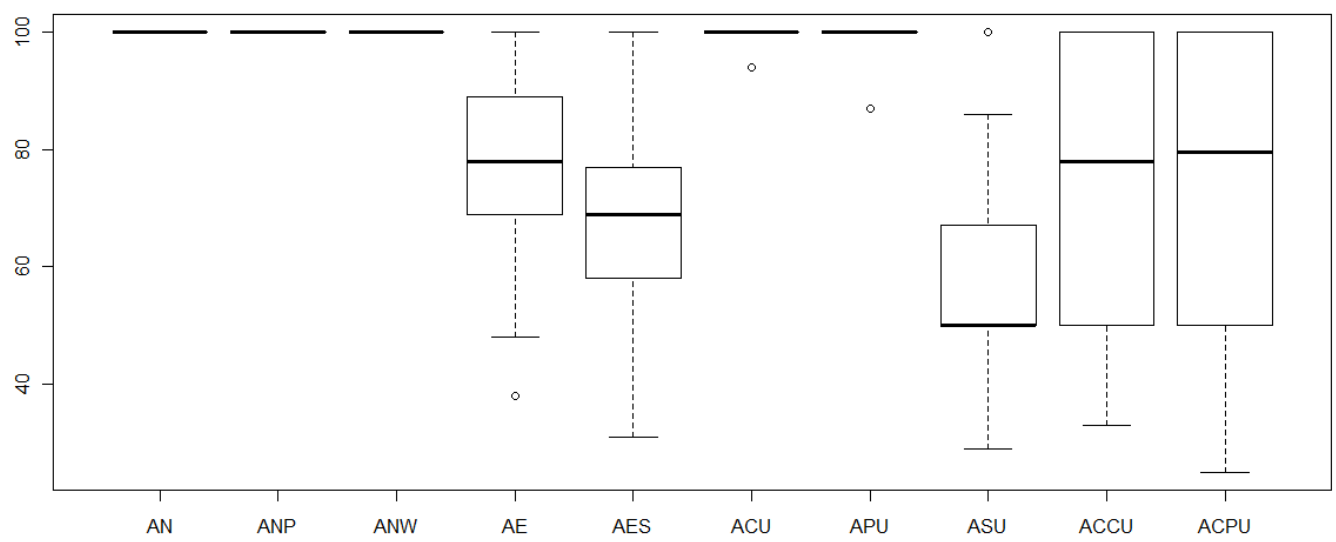

Figura 4.9: Dificuldade de satisfação para o critério all-nodes quando aplicado aos demais critérios.

Ao aplicar o conjunto de testes adequado ao critério all-w-nodes (ANW) os resultados obtidos para o strength dos critérios foi bastante variado, conforme apresentado na Figura 


\section{CAPÍTULO 4. ANÁLISE DOS RESULTADOS}

Tabela 4.6: Aplicação do conjunto de casos de teste adequado ao critério all-p-nodes (ANP) aos demais critérios.

\begin{tabular}{|c|c|c|c|c|c|c|c|c|c|}
\hline Programas & $\mathbf{A N}$ & ANW & $\mathbf{A E}$ & AES & ACU & APU & ASU & ACCU & ACPU \\
\hline Sleeping barber & $100 \%$ & $100 \%$ & $57 \%$ & $44 \%$ & $100 \%$ & $*$ & $38 \%$ & $100 \%$ & $100 \%$ \\
\hline GCD & $100 \%$ & $100 \%$ & $69 \%$ & $67 \%$ & $100 \%$ & * & $50 \%$ & $50 \%$ & $*$ \\
\hline Jacobi & $100 \%$ & $100 \%$ & $85 \%$ & $75 \%$ & $94 \%$ & $87 \%$ & $75 \%$ & $100 \%$ & $50 \%$ \\
\hline MMult & $100 \%$ & $100 \%$ & $71 \%$ & $57 \%$ & $100 \%$ & $100 \%$ & $50 \%$ & $100 \%$ & $100 \%$ \\
\hline ProdCons & $100 \%$ & $100 \%$ & $78 \%$ & $71 \%$ & $100 \%$ & * & $50 \%$ & $33 \%$ & * \\
\hline Carter01 & $100 \%$ & $100 \%$ & $68 \%$ & $60 \%$ & $100 \%$ & $*$ & $33 \%$ & $50 \%$ & $33 \%$ \\
\hline Deadlock01 & $100 \%$ & $100 \%$ & $38 \%$ & $31 \%$ & $100 \%$ & * & $36 \%$ & * & $*$ \\
\hline Dpor-example1 & $100 \%$ & $100 \%$ & $69 \%$ & $60 \%$ & $100 \%$ & * & $60 \%$ & $50 \%$ & $*$ \\
\hline Dpor-example2 & $100 \%$ & $100 \%$ & $100 \%$ & $100 \%$ & $100 \%$ & * & $100 \%$ & $100 \%$ & * \\
\hline Dpor-example3 & $100 \%$ & $100 \%$ & $82 \%$ & $75 \%$ & $100 \%$ & $*$ & $50 \%$ & * & * \\
\hline Lazy01 & $100 \%$ & $100 \%$ & $53 \%$ & $43 \%$ & $100 \%$ & * & $33 \%$ & $50 \%$ & $*$ \\
\hline Phase01 & $100 \%$ & $100 \%$ & $85 \%$ & $75 \%$ & $100 \%$ & $100 \%$ & $67 \%$ & $100 \%$ & $*$ \\
\hline Race01 & $100 \%$ & $100 \%$ & $64 \%$ & $54 \%$ & $100 \%$ & * & $50 \%$ & $50 \%$ & * \\
\hline Rafkind01 & $100 \%$ & $100 \%$ & $73 \%$ & $67 \%$ & $100 \%$ & * & $55 \%$ & * & * \\
\hline Simple1 & $100 \%$ & $100 \%$ & $83 \%$ & $75 \%$ & $100 \%$ & * & $50 \%$ & * & $*$ \\
\hline Stateful01 & $100 \%$ & $100 \%$ & $69 \%$ & $60 \%$ & $100 \%$ & * & $60 \%$ & $50 \%$ & * \\
\hline Stateful06 & $100 \%$ & $100 \%$ & $100 \%$ & $100 \%$ & $100 \%$ & * & * & * & * \\
\hline Hg01_all_ok & $100 \%$ & $100 \%$ & $83 \%$ & $75 \%$ & $*$ & $100 \%$ & $50 \%$ & $50 \%$ & * \\
\hline Hg02_deadlock & $100 \%$ & $100 \%$ & $100 \%$ & $100 \%$ & * & $100 \%$ & $100 \%$ & $100 \%$ & * \\
\hline Hg03_inherit & $100 \%$ & $100 \%$ & $80 \%$ & $71 \%$ & * & $100 \%$ & $50 \%$ & $50 \%$ & $50 \%$ \\
\hline Hg04_race & $100 \%$ & $100 \%$ & $54 \%$ & $40 \%$ & * & $100 \%$ & $50 \%$ & $50 \%$ & $50 \%$ \\
\hline Hg06_readshare & * & * & * & * & * & * & * & $*$ & $*$ \\
\hline Tc01_simple_race & $100 \%$ & $100 \%$ & $100 \%$ & $100 \%$ & $100 \%$ & $100 \%$ & $*$ & $*$ & $*$ \\
\hline Tc02_simple_tls & $100 \%$ & $100 \%$ & $100 \%$ & $100 \%$ & * & * & $100 \%$ & * & * \\
\hline Tc05_simple_race & $100 \%$ & $100 \%$ & $73 \%$ & $60 \%$ & $100 \%$ & $100 \%$ & $33 \%$ & $100 \%$ & $67 \%$ \\
\hline Tc06_two_races & $100 \%$ & $100 \%$ & $48 \%$ & $38 \%$ & $100 \%$ & $100 \%$ & $29 \%$ & * & $25 \%$ \\
\hline Tc13_laog & $90 \%$ & $100 \%$ & $95 \%$ & $100 \%$ & $45 \%$ & $60 \%$ & $57 \%$ & $100 \%$ & $100 \%$ \\
\hline Tc16_byterace & $91 \%$ & $100 \%$ & $83 \%$ & $75 \%$ & $45 \%$ & $58 \%$ & $33 \%$ & $50 \%$ & $100 \%$ \\
\hline Tc18_semabuse & $100 \%$ & $100 \%$ & $55 \%$ & $47 \%$ & $100 \%$ & $100 \%$ & $36 \%$ & $100 \%$ & $100 \%$ \\
\hline Account & $81 \%$ & $100 \%$ & $60 \%$ & $51 \%$ & $65 \%$ & $42 \%$ & $50 \%$ & $55 \%$ & $100 \%$ \\
\hline Reorder & $97 \%$ & $100 \%$ & $85 \%$ & $80 \%$ & $95 \%$ & $90 \%$ & $73 \%$ & $78 \%$ & $77 \%$ \\
\hline Twostage & $100 \%$ & $100 \%$ & $72 \%$ & $59 \%$ & $100 \%$ & $100 \%$ & $53 \%$ & $78 \%$ & * \\
\hline Wronglock & $94 \%$ & $100 \%$ & $72 \%$ & $64 \%$ & $100 \%$ & $100 \%$ & $59 \%$ & $47 \%$ & $50 \%$ \\
\hline
\end{tabular}

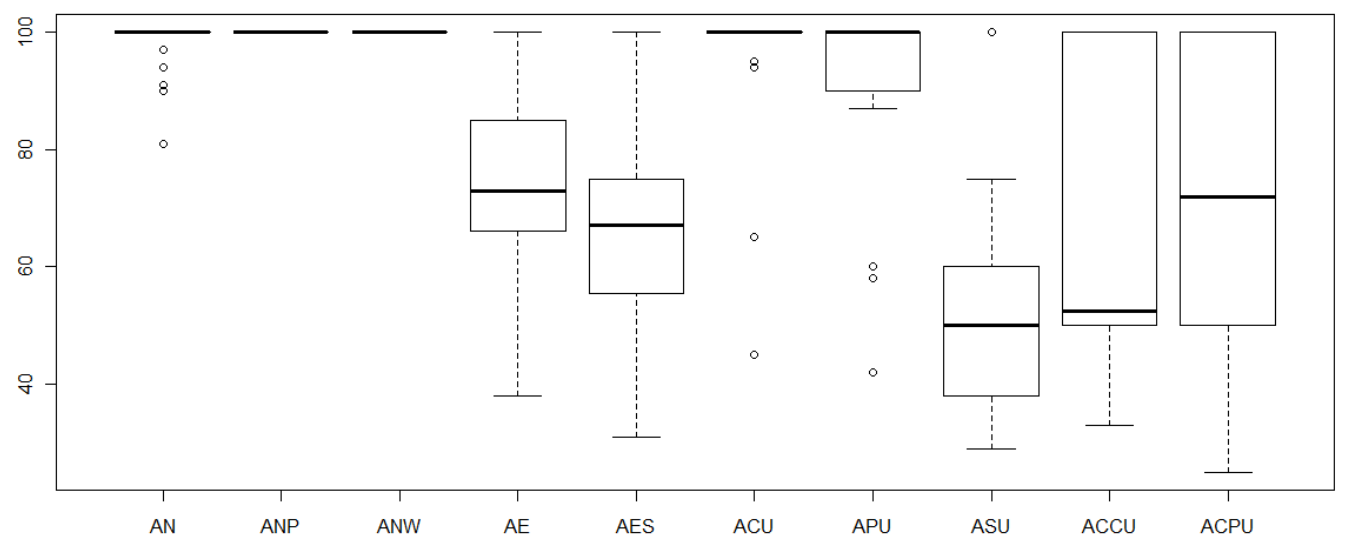

Figura 4.10: Dificuldade de satisfação para o critério all-p-nodes quando aplicado aos demais critérios.

4.11. Os critérios all-comm-c-uses (ACCU), all-comm-p-uses (ACPU), all-edges (AE), all-s-edges (AES), e all-sync-uses (ASU) apresentarem um índice alto de dificuldade de 


\section{CAPÍTULO 4. ANÁLISE DOS RESULTADOS}

Tabela 4.7: Aplicação do conjunto de casos de teste adequado ao critério all-w-nodes (ANW) aos demais critérios.

\begin{tabular}{|c|c|c|c|c|c|c|c|c|c|}
\hline Programas & $\mathbf{A N}$ & ANP & $\mathbf{A E}$ & AES & $\mathrm{ACU}$ & APU & ASU & ACCU & ACPU \\
\hline Sleeping barber & $100 \%$ & $100 \%$ & $57 \%$ & $44 \%$ & $100 \%$ & $*$ & $38 \%$ & $100 \%$ & $100 \%$ \\
\hline GCD & $100 \%$ & $100 \%$ & $69 \%$ & $67 \%$ & $100 \%$ & * & $50 \%$ & $50 \%$ & * \\
\hline Jacobi & $100 \%$ & $100 \%$ & $85 \%$ & $75 \%$ & $94 \%$ & $87 \%$ & $75 \%$ & $100 \%$ & $50 \%$ \\
\hline MMult & $100 \%$ & $100 \%$ & $71 \%$ & $57 \%$ & $100 \%$ & $100 \%$ & $5 \%$ & $100 \%$ & $100 \%$ \\
\hline ProdCons & $100 \%$ & $100 \%$ & $78 \%$ & $71 \%$ & $100 \%$ & $*$ & $50 \%$ & $33 \%$ & $*$ \\
\hline Carter01 & $100 \%$ & $100 \%$ & $68 \%$ & $6 \%$ & $100 \%$ & * & $33 \%$ & $50 \%$ & $33 \%$ \\
\hline Deadlock01 & $100 \%$ & $100 \%$ & $38 \%$ & $31 \%$ & $100 \%$ & * & $36 \%$ & $*$ & $*$ \\
\hline Dpor-example1 & $100 \%$ & $100 \%$ & $69 \%$ & $60 \%$ & $100 \%$ & * & $60 \%$ & $50 \%$ & * \\
\hline Dpor-example2 & $100 \%$ & $100 \%$ & $100 \%$ & $100 \%$ & $100 \%$ & $*$ & $100 \%$ & $100 \%$ & * \\
\hline Dpor-example3 & $100 \%$ & $100 \%$ & $82 \%$ & $75 \%$ & $100 \%$ & $*$ & $50 \%$ & $*$ & $*$ \\
\hline Lazy01 & $100 \%$ & $100 \%$ & $53 \%$ & $43 \%$ & $100 \%$ & $*$ & $33 \%$ & $50 \%$ & * \\
\hline Phase01 & $100 \%$ & $100 \%$ & $85 \%$ & $75 \%$ & $100 \%$ & $100 \%$ & $67 \%$ & $100 \%$ & * \\
\hline Race01 & $100 \%$ & $100 \%$ & $64 \%$ & $54 \%$ & $100 \%$ & $*$ & $50 \%$ & $50 \%$ & $*$ \\
\hline Rafkind01 & $100 \%$ & $100 \%$ & $73 \%$ & $67 \%$ & $100 \%$ & $*$ & $55 \%$ & * & $*$ \\
\hline Simple1 & $100 \%$ & $100 \%$ & $83 \%$ & $75 \%$ & $100 \%$ & $*$ & $50 \%$ & * & $*$ \\
\hline Stateful01 & $100 \%$ & $100 \%$ & $69 \%$ & $60 \%$ & $100 \%$ & $*$ & $60 \%$ & $50 \%$ & * \\
\hline Stateful06 & $100 \%$ & $100 \%$ & $100 \%$ & $100 \%$ & $100 \%$ & $*$ & * & * & * \\
\hline Hg01_all_ok & $100 \%$ & $100 \%$ & $83 \%$ & $75 \%$ & * & $100 \%$ & $50 \%$ & $50 \%$ & * \\
\hline Hg02_deadlock & $100 \%$ & $100 \%$ & $100 \%$ & $100 \%$ & * & $100 \%$ & $100 \%$ & $100 \%$ & * \\
\hline Hg03_inherit & $100 \%$ & $100 \%$ & $80 \%$ & $71 \%$ & * & $100 \%$ & $50 \%$ & $50 \%$ & $50 \%$ \\
\hline Hg04_race & $100 \%$ & $100 \%$ & $54 \%$ & $40 \%$ & * & $100 \%$ & $50 \%$ & $50 \%$ & $50 \%$ \\
\hline Hg06_readshare & $*$ & $*$ & $*$ & $*$ & * & $*$ & * & * & $*$ \\
\hline Tc01_simple_race & $100 \%$ & $100 \%$ & $100 \%$ & $100 \%$ & $100 \%$ & $100 \%$ & * & $*$ & $*$ \\
\hline Tc02_simple_tls & $100 \%$ & $100 \%$ & $100 \%$ & $100 \%$ & * & * & $100 \%$ & * & * \\
\hline Tc05_simple_race & $100 \%$ & $100 \%$ & $73 \%$ & $60 \%$ & $100 \%$ & $100 \%$ & $33 \%$ & $100 \%$ & $67 \%$ \\
\hline Tc06_two_races & $100 \%$ & $100 \%$ & $48 \%$ & $38 \%$ & $100 \%$ & $100 \%$ & $29 \%$ & * & $25 \%$ \\
\hline Tc13_laog & $84 \%$ & $73 \%$ & $71 \%$ & $67 \%$ & $45 \%$ & $58 \%$ & $29 \%$ & $100 \%$ & $50 \%$ \\
\hline Tc16_byterace & $84 \%$ & $88 \%$ & $78 \%$ & $75 \%$ & $45 \%$ & $54 \%$ & $33 \%$ & $50 \%$ & $100 \%$ \\
\hline Tc18_semabuse & $100 \%$ & $100 \%$ & $55 \%$ & $47 \%$ & $100 \%$ & $100 \%$ & $36 \%$ & $100 \%$ & $100 \%$ \\
\hline Account & $95 \%$ & $100 \%$ & $76 \%$ & $64 \%$ & $78 \%$ & $100 \%$ & $63 \%$ & $86 \%$ & $100 \%$ \\
\hline Reorder & $96 \%$ & $96 \%$ & $66 \%$ & $42 \%$ & $100 \%$ & $100 \%$ & $40 \%$ & $83 \%$ & $60 \%$ \\
\hline Twostage & $100 \%$ & $100 \%$ & $72 \%$ & $59 \%$ & $100 \%$ & $100 \%$ & $53 \%$ & $78 \%$ & * \\
\hline Wronglock & $94 \%$ & $100 \%$ & $72 \%$ & $64 \%$ & $100 \%$ & $100 \%$ & $59 \%$ & $47 \%$ & $50 \%$ \\
\hline
\end{tabular}

satisfação, já os critérios all-nodes (AN), all-p-nodes (ANP), all-c-uses (ACU) e all-p-uses (APU) apresentaram, em média, valores baixos de strength, porém muitos outliers, o que torna impossível tirar conclusões a respeito dos dados antes do teste estatístico e redução dos dados.

A Figura 4.12 apresenta os dados da aplicação dos conjunto adequado ao critério all-edges (AE) aos demais critérios avaliados. De maneira geral, os critérios obtiveram baixo strength quando submetidos ao conjunto adequado ao critério all-edges, o que indica que o critério all-edges é capaz de incluir a maioria dos critérios. Um possível motivo para isso é que o critério all-edges inclui os requisitos de teste do critério all-s-edges, pois esse resultado não é similar aqueles normalmente obtidos para programas sequenciais. Uma atenção especial deve ser dada, porém, aos critérios all-sync-uses (ASU), all-comm-c-uses (ACCU) e all-comm-p-uses que apresentam alguns outliers para a amostra.

Para o critério all-s-edges (AES) foi gerado o gráfico boxplot ilustrado na Figura 4.13. O gráfico sugere que os critérios all-nodes (AN), all-p-nodes (ANP) e all-w-nodes (ANW) apresentam baixo strength para o conjunto adequado ao critério all-s-edges. Em relação 


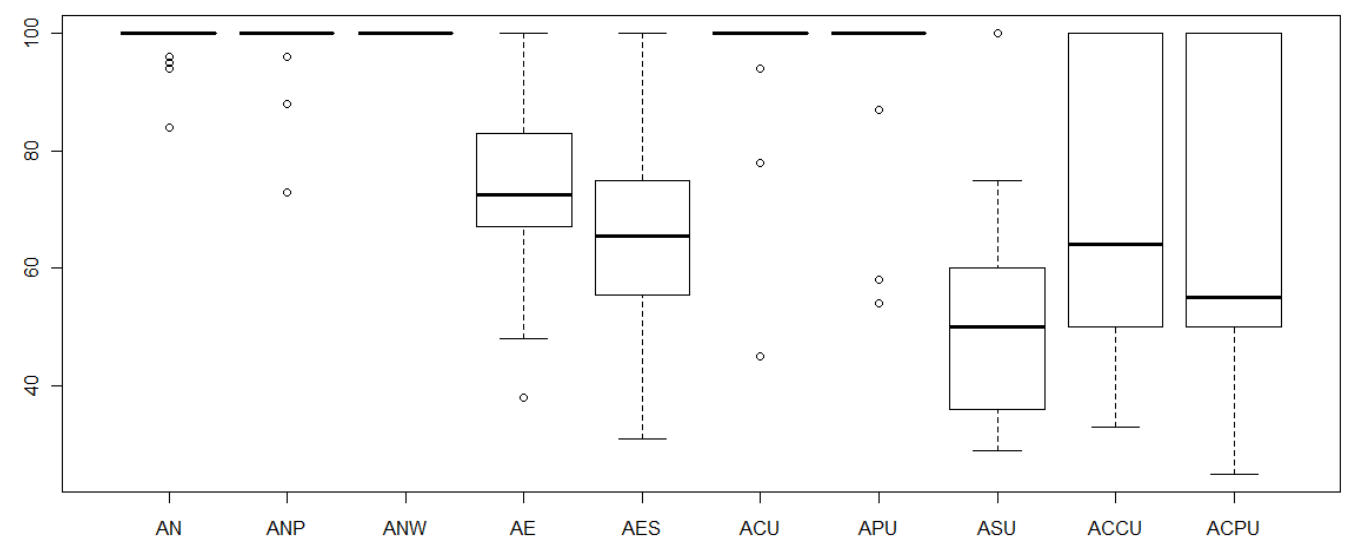

Figura 4.11: Dificuldade de satisfação para o critério all-w-nodes quando aplicado aos demais critérios

Tabela 4.8: Aplicação do conjunto de casos de teste adequado ao critério all-edges (AE) aos demais critérios.

\begin{tabular}{|c|c|c|c|c|c|c|c|c|c|}
\hline Programa & $\mathbf{A N}$ & ANP & ANW & AES & ACU & APU & ASU & ACCU & ACPU \\
\hline Sleeping barber & $100 \%$ & $100 \%$ & $100 \%$ & $100 \%$ & * & * & $100 \%$ & $*$ & $*$ \\
\hline GCD & $100 \%$ & $100 \%$ & $100 \%$ & $100 \%$ & $100 \%$ & $*$ & $100 \%$ & $100 \%$ & * \\
\hline Jacobi & $100 \%$ & $100 \%$ & $100 \%$ & $100 \%$ & $100 \%$ & * & $100 \%$ & $100 \%$ & $100 \%$ \\
\hline Mmult & $100 \%$ & $100 \%$ & $100 \%$ & $100 \%$ & $100 \%$ & $100 \%$ & $100 \%$ & * & $*$ \\
\hline ProdCons & $100 \%$ & $100 \%$ & $100 \%$ & $100 \%$ & $100 \%$ & * & $100 \%$ & $100 \%$ & * \\
\hline Carter01 & $100 \%$ & $100 \%$ & $100 \%$ & $100 \%$ & $100 \%$ & $100 \%$ & $100 \%$ & * & $*$ \\
\hline Deadlock01 & $100 \%$ & $100 \%$ & $100 \%$ & $100 \%$ & $100 \%$ & $100 \%$ & $100 \%$ & $100 \%$ & * \\
\hline Dpor-example1 & $100 \%$ & $100 \%$ & $100 \%$ & $100 \%$ & $100 \%$ & * & * & $100 \%$ & * \\
\hline Dpor-example2 & $100 \%$ & $100 \%$ & $100 \%$ & $100 \%$ & $*$ & $100 \%$ & $100 \%$ & $100 \%$ & $*$ \\
\hline Dpor-example3 & $100 \%$ & $100 \%$ & $100 \%$ & $100 \%$ & $100 \%$ & $100 \%$ & $*$ & $100 \%$ & $97 \%$ \\
\hline Lazy01 & $100 \%$ & $100 \%$ & $100 \%$ & $100 \%$ & $100 \%$ & * & $100 \%$ & $100 \%$ & * \\
\hline Phase01 & $100 \%$ & $100 \%$ & $100 \%$ & $100 \%$ & $100 \%$ & $100 \%$ & $100 \%$ & $98 \%$ & * \\
\hline Race01 & $98 \%$ & $100 \%$ & $100 \%$ & $97 \%$ & $94 \%$ & $98 \%$ & * & $*$ & * \\
\hline Rafkind01 & $100 \%$ & $100 \%$ & $100 \%$ & $100 \%$ & $100 \%$ & * & $100 \%$ & $100 \%$ & * \\
\hline Simple1 & $100 \%$ & $100 \%$ & $100 \%$ & $100 \%$ & $100 \%$ & * & $100 \%$ & * & $*$ \\
\hline Stateful01 & $100 \%$ & $100 \%$ & $100 \%$ & $100 \%$ & * & $100 \%$ & $100 \%$ & $100 \%$ & * \\
\hline Stateful06 & $100 \%$ & $100 \%$ & $100 \%$ & $100 \%$ & $100 \%$ & $100 \%$ & $97 \%$ & * & $*$ \\
\hline Hg01_all_ok & $100 \%$ & $100 \%$ & $100 \%$ & $100 \%$ & * & $100 \%$ & $*$ & $100 \%$ & * \\
\hline Hg02_deadlock & $100 \%$ & $100 \%$ & $100 \%$ & $100 \%$ & $6 \%$ & $62 \%$ & $33 \%$ & $100 \%$ & $91 \%$ \\
\hline Hg03_inherit & $100 \%$ & $100 \%$ & $100 \%$ & $100 \%$ & * & $100 \%$ & $*$ & $100 \%$ & $100 \%$ \\
\hline Hg04_race & $100 \%$ & $100 \%$ & $100 \%$ & $100 \%$ & $100 \%$ & $*$ & $100 \%$ & $100 \%$ & $100 \%$ \\
\hline Hg06_readshared & $100 \%$ & $100 \%$ & $100 \%$ & $100 \%$ & $100 \%$ & $100 \%$ & $100 \%$ & $100 \%$ & $100 \%$ \\
\hline Tc01_simple_race & $100 \%$ & $100 \%$ & $100 \%$ & $100 \%$ & $100 \%$ & $100 \%$ & $100 \%$ & $*$ & $100 \%$ \\
\hline Tc02_simple_tls & $100 \%$ & $100 \%$ & $100 \%$ & $100 \%$ & $100 \%$ & * & * & $*$ & * \\
\hline Tc05_simple_race & $100 \%$ & $100 \%$ & $100 \%$ & $100 \%$ & $100 \%$ & * & $100 \%$ & $100 \%$ & $*$ \\
\hline Tc06_two_races & $100 \%$ & $100 \%$ & $100 \%$ & $100 \%$ & $100 \%$ & $100 \%$ & $100 \%$ & $100 \%$ & $100 \%$ \\
\hline Tc13_laog1 & $100 \%$ & $100 \%$ & $100 \%$ & $100 \%$ & $100 \%$ & $*$ & $100 \%$ & $100 \%$ & $*$ \\
\hline Tc16_byterace & $100 \%$ & $100 \%$ & $100 \%$ & $100 \%$ & $100 \%$ & * & $100 \%$ & * & * \\
\hline Tc18_semabuse & $100 \%$ & $*$ & $100 \%$ & * & $100 \%$ & * & * & * & * \\
\hline Account & $100 \%$ & $100 \%$ & $100 \%$ & $100 \%$ & $100 \%$ & $100 \%$ & $*$ & * & * \\
\hline Reorder & $100 \%$ & $100 \%$ & $100 \%$ & $100 \%$ & $100 \%$ & * & $100 \%$ & * & * \\
\hline Twostage & $96 \%$ & $85 \%$ & $100 \%$ & $100 \%$ & $100 \%$ & $61 \%$ & $100 \%$ & $100 \%$ & $100 \%$ \\
\hline Wronglock & $100 \%$ & $100 \%$ & $100 \%$ & $100 \%$ & $100 \%$ & * & $*$ & $*$ & $*$ \\
\hline
\end{tabular}

aos demais critérios nenhuma conclusão pode ser obtida antes da análise dos outlieres identificados na amostra na fase de redução do conjunto de dados. 


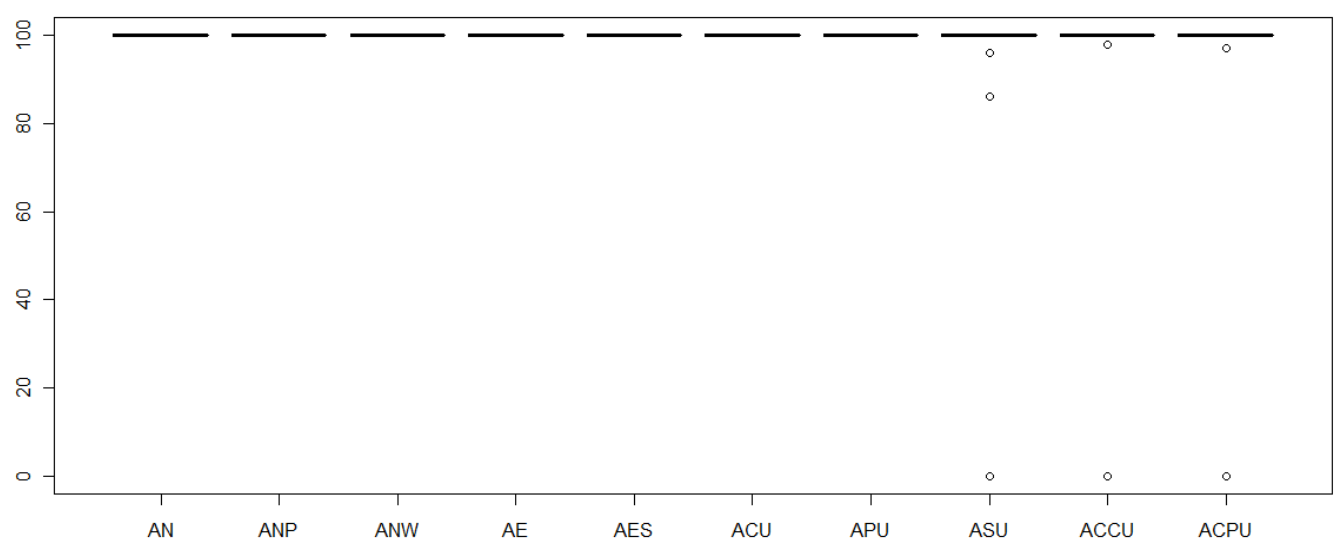

Figura 4.12: Dificuldade de satisfação para o critério all-edges quando aplicado aos demais critérios.

Tabela 4.9: Aplicação do conjunto de casos de teste adequado ao critério all-s-edges (AES) aos demais critérios.

\begin{tabular}{|c|c|c|c|c|c|c|c|c|}
\hline Programa & $\mathbf{A N}$ & ANP & ANW & AE & $\mathrm{ACU}$ & APU & ASU & $\mathrm{ACCU}$ \\
\hline Sleeping barber & $95 \%$ & $78 \%$ & $100 \%$ & $87 \%$ & $56 \%$ & $52 \%$ & $67 \%$ & $100 \%$ \\
\hline GCD & $96 \%$ & $85 \%$ & $100 \%$ & $100 \%$ & $100 \%$ & $61 \%$ & $100 \%$ & $100 \%$ \\
\hline Jacobi & $100 \%$ & $100 \%$ & $100 \%$ & $100 \%$ & $100 \%$ & $100 \%$ & $100 \%$ & $*$ \\
\hline Mmult & $100 \%$ & $100 \%$ & $100 \%$ & $100 \%$ & $100 \%$ & $*$ & $100 \%$ & $100 \%$ \\
\hline ProdCons & $100 \%$ & $100 \%$ & $100 \%$ & $100 \%$ & $*$ & $100 \%$ & $100 \%$ & $100 \%$ \\
\hline Carter01 & $100 \%$ & $100 \%$ & $100 \%$ & $100 \%$ & $100 \%$ & $*$ & $100 \%$ & $*$ \\
\hline Deadlock01 & $*$ & $*$ & $*$ & $*$ & $*$ & $*$ & $*$ & $*$ \\
\hline Dpor-example1 & $100 \%$ & $100 \%$ & $100 \%$ & $100 \%$ & $100 \%$ & $100 \%$ & $*$ & $100 \%$ \\
\hline Dpor-example2 & $100 \%$ & $100 \%$ & $100 \%$ & $100 \%$ & $*$ & $100 \%$ & $*$ & $100 \%$ \\
\hline Dpor-example3 & $93 \%$ & $70 \%$ & $100 \%$ & $82 \%$ & $100 \%$ & $92 \%$ & $100 \%$ & $*$ \\
\hline Lazy01 & $100 \%$ & $100 \%$ & $100 \%$ & $100 \%$ & $100 \%$ & * & $100 \%$ & $100 \%$ \\
\hline Phase01 & $100 \%$ & $100 \%$ & $100 \%$ & $100 \%$ & $100 \%$ & $*$ & $*$ & $*$ \\
\hline Race01 & $100 \%$ & $100 \%$ & $100 \%$ & $100 \%$ & $100 \%$ & $*$ & $*$ & $*$ \\
\hline Rafkind01 & $100 \%$ & $100 \%$ & $100 \%$ & $100 \%$ & $100 \%$ & $100 \%$ & $*$ & * \\
\hline Simple1 & $100 \%$ & $100 \%$ & $100 \%$ & $100 \%$ & $*$ & $100 \%$ & * & $100 \%$ \\
\hline Stateful01 & $100 \%$ & $100 \%$ & $100 \%$ & $100 \%$ & $100 \%$ & $*$ & $100 \%$ & $100 \%$ \\
\hline Stateful06 & $100 \%$ & $100 \%$ & $100 \%$ & $100 \%$ & $100 \%$ & $100 \%$ & $100 \%$ & 98 \\
\hline Hg01_all_ok & $100 \%$ & $100 \%$ & $100 \%$ & $100 \%$ & $100 \%$ & $*$ & $100 \%$ & $*$ \\
\hline Hg02_deadlock & $100 \%$ & $100 \%$ & $100 \%$ & $100 \%$ & $*$ & $100 \%$ & $100 \%$ & $100 \%$ \\
\hline Hg03_inherit & $100 \%$ & $100 \%$ & $100 \%$ & $100 \%$ & $100 \%$ & $*$ & $100 \%$ & $*$ \\
\hline Hg04_race & $100 \%$ & $100 \%$ & $100 \%$ & $100 \%$ & $100 \%$ & $100 \%$ & $100 \%$ & $100 \%$ \\
\hline Hg06_readshared & $100 \%$ & $100 \%$ & $100 \%$ & $100 \%$ & $100 \%$ & $100 \%$ & $100 \%$ & $*$ \\
\hline Tc01_simple_race & $100 \%$ & $100 \%$ & $100 \%$ & $100 \%$ & $100 \%$ & $100 \%$ & $100 \%$ & $*$ \\
\hline Tc02_simple_tls & $100 \%$ & $100 \%$ & $100 \%$ & $100 \%$ & $100 \%$ & * & $100 \%$ & $100 \%$ \\
\hline Tc05_simple_race & $98 \%$ & $100 \%$ & $100 \%$ & $100 \%$ & $94 \%$ & $98 \%$ & $*$ & $*$ \\
\hline Tc06_two_races & $100 \%$ & $100 \%$ & $100 \%$ & $100 \%$ & * & $*$ & $100 \%$ & $*$ \\
\hline Tc13_laog1 & $100 \%$ & $100 \%$ & $100 \%$ & $100 \%$ & $100 \%$ & $*$ & $100 \%$ & $100 \%$ \\
\hline Tc16_byterace & $100 \%$ & $100 \%$ & $100 \%$ & $100 \%$ & $100 \%$ & $*$ & $100 \%$ & $100 \%$ \\
\hline Tc18_semabuse & $100 \%$ & $100 \%$ & $100 \%$ & $100 \%$ & $100 \%$ & $100 \%$ & $100 \%$ & $100 \%$ \\
\hline Account & $100 \%$ & $100 \%$ & $100 \%$ & $100 \%$ & $100 \%$ & $*$ & $100 \%$ & $100 \%$ \\
\hline Reorder & $100 \%$ & $100 \%$ & $100 \%$ & $100 \%$ & $100 \%$ & * & $*$ & $100 \%$ \\
\hline Twostage & $100 \%$ & $100 \%$ & $100 \%$ & $100 \%$ & $100 \%$ & * & $100 \%$ & $100 \%$ \\
\hline Wronglock & $95 \%$ & $100 \%$ & $100 \%$ & $100 \%$ & $78 \%$ & $76 \%$ & $100 \%$ & $100 \%$ \\
\hline
\end{tabular}

Com a análise dos dados coletados para o fator strength observou-se um número elevado de outliers. Os outliers, em geral, apresentam dados que, por ter um comportamento 


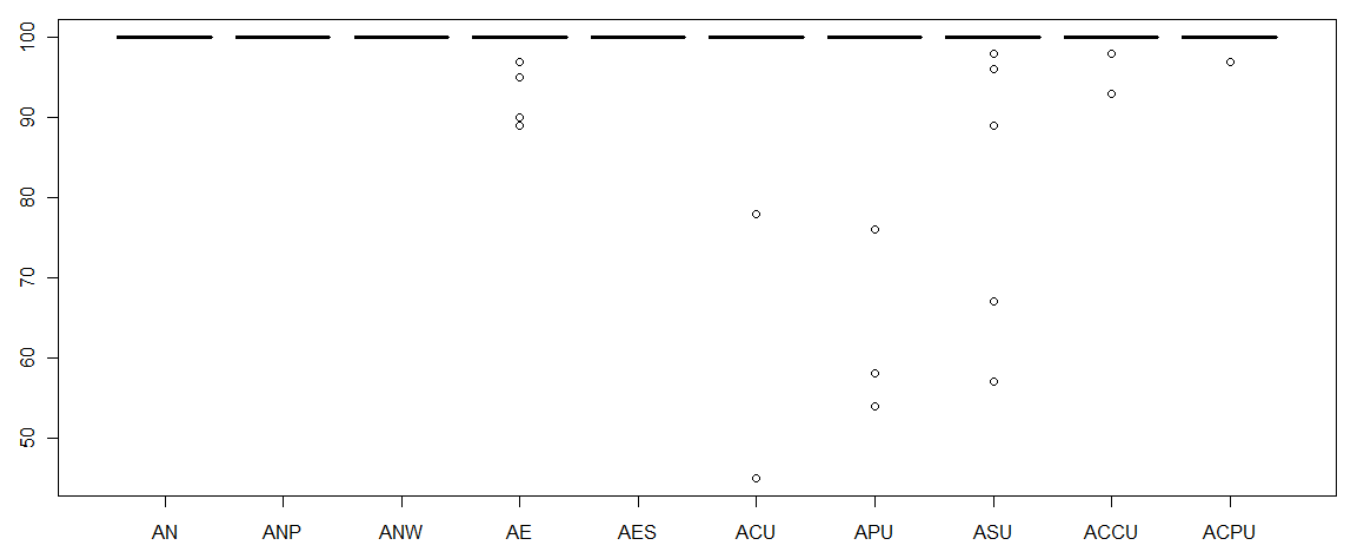

Figura 4.13: Dificuldade de satisfação para o critério all-s-edges quando aplicado aos demais critérios.

diferente do conjunto, devem ser investigados. Se provado que esses valores aparentemente anormais provém de erros na análise dos dados, eles podem ser descartados e o teste estatístico segue normalmente. Caso contrário, os outliers devem ser cuidadosamente analisados, pois podem conter informações relevantes sobre a população que podem ser decisivas nas análises realizadas e talvez constituírem o fator de interesse a ser estudado. Na próxima seção é apresentada a redução dos dados, obtida com a análise de outliers para esta amostra.

\subsection{Redução do Conjunto de Dados}

$\mathrm{Na}$ fase de análise dos dados utilizando a estatística descritiva, outliers foram identificados na amostra utilizada. A análise da origem desses valores "anormais" mostrou que eles pertencem aos resultados obtidos com os programas mais complexos que compunham a amostra, ou seja, os programas provenientes do benchmark Rungta e os programas que implementam soluções para problemas clássicos de programação concorrente. No caso deste experimento em particular, um programa é dito mais complexo se apresenta um maior número de variáveis compartilhadas e variáveis de sincronização além de uma maior quantidade de linhas de código. Esses valores influenciam os resultados para a amostra e simplesmente descartá-los não seria aconselhável. Comparações entre os dados mostraram que os valores outliers, são na verdade, os valores de interesse dessa pesquisa, pois compreendem o conjunto de programas com mais resultados significativos para os critérios avaliados. Optou-se, então, por não considerar no teste de hipótese os programas provenientes dos benchmarks Inspect e Helgrind, pois por serem mais simples não apresentam elementos para alguns critérios avaliados. 


\subsection{Teste de Hipóteses}

\subsubsection{Custo do Critério de Teste}

Existem diferentes perspectivas para a avaliação do custo de um critério de teste. Neste estudo optamos por mensurá-lo com base no número de casos de testes necessários para satisfação do critério (conjunto adequado), o número de elementos requeridos para cada critério e o número de elementos não-executáveis. A hipótese nula (HN1), definida anteriormente, que afirma que não há diferença significativa de custo entre os critérios avaliados, é rejeitada se, por meio dos testes estatísticos, for comprovado que para qualquer um destes fatores, quaisquer dois critérios distintos apresentam diferença significativa de custo entre si.

A análise do custo considerando a quantidade de casos de teste que compõe o conjunto de casos de teste adequado inciou-se com o teste de Shapiro-Wilk a fim de verificar a normalidade dos dados. O teste resultou em um valor de $\mathrm{p}$-value $=0.005915$, portanto, menor que 0.05 provando que essa amostra não é normal e que o teste estatístico a ser usado é o teste não-paramétrico e optamos por usar o teste de Kruskal-Wallis a fim de testar a hipótese. O resultado do teste foi de p-value $=0.0001914$, provando que a hipótese nula (HN1) pode ser rejeitada, quando consideramos o tamanho do conjunto adequado. Ou seja, que existe ao menos um par de critérios para os quais há uma diferença significativa nos valores para o custo considerando o conjunto adequado.

A próxima etapa do teste estatístico é verificar para quais pares de critérios a hipótese alternativa deve ser aceita, sendo que para esta análise foi utilizado também o teste Kruskal-Wallis. Os resultados para o teste Kruskal-Wallis são apresentados na Tabela 4.10. Na tabela, a primeira coluna corresponde aos pares de fatores comparados, a segunda coluna apresenta o valor da diferença média entre os pares da amostra, a terceira coluna apresenta o valor base para a diferença crítica. Se o valor da diferença média observada for maior ou igual ao valor da diferença crítica, há indícios de que há diferença significativa entre os critérios comparados. Quando há diferença significativa entre os pares, a quarta coluna da tabela apresenta o valor "TRUE", e quando não há diferença, apresenta o valor "FALSE". Observando a tabela pode-se concluir que para o par de critérios all-edges (AE) e all-w-nodes (ANW) e também para o par de critérios critérios all-sync-uses (ASU) e all-w-nodes (ANW) há uma diferença significativa de custo.

A análise do teste de normalidade para o custo considerando a quantidade de elementos requeridos foi realizada usando o teste de Shapiro-Wilk, que resultou em um p-value $=0.2909$ o que prova que esta amostra é normal. O teste de hipótese usado foi o teste de análise de variância ANOVA, o resultado do teste indica que podemos rejeitar a hipótese 
Tabela 4.10: Resultados do teste Kruskal-Wallis para o custo em relação ao conjunto de casos de teste adequado.

\begin{tabular}{|c|c|c|c|}
\hline \multicolumn{4}{|c|}{$\begin{array}{l}\text { Multiple comparison test after Kruskal-Wallis p.value: } 0.0 \\
\text { Comparisons }\end{array}$} \\
\hline & obs.dif & critical.dif & difference \\
\hline ACCU-ACPU & 7.6875000 & 41.65197 & FALSE \\
\hline ACCU-ACU & 8.9861111 & 40.47844 & FALSE \\
\hline ACCU-AE & 25.3472222 & 40.47844 & FALSE \\
\hline ACCU-AES & 20.7916667 & 40.47844 & FALSE \\
\hline ACCU-AN & 3.5416667 & 40.47844 & FALSE \\
\hline ACCU-ANP & 12.1527778 & 40.47844 & FALSE \\
\hline ACCU-ANW & 15.2083333 & 40.47844 & FALSE \\
\hline ACCU-APU & 8.3750000 & 40.47844 & FALSE \\
\hline ACCU-ASU & 24.8472222 & 40.47844 & FALSE \\
\hline ACPU-ACU & 12.986111 & 40.47844 & FALSE \\
\hline ACPU-AE & 33.0347222 & 40.47844 & FALSE \\
\hline ACPU-AES & 28.4791667 & 40.47844 & FALSE \\
\hline ACPU-AN & 4.1458333 & 40.47844 & FALSE \\
\hline ACPU-ANP & 4.4652778 & 40.47844 & FALSE \\
\hline ACPU-ANW & 7.5208333 & 40.47844 & FALSE \\
\hline ACPU-APU & 0.6875000 & 40.47844 & FALSE \\
\hline ACPU-ASU & 32.5347222 & 40.47844 & FALSE \\
\hline ACU-AE & 34.3333333 & 39.26985 & FALSE \\
\hline ACU-AES & 29.7777778 & 39.26985 & FALSE \\
\hline ACU-AN & 5.4444444 & 39.26985 & FALSE \\
\hline ACU-ANP & 3.1666667 & 39.26985 & FALSE \\
\hline ACU-ANW & 62.222 .222 & 39.26985 & FALSE \\
\hline ACU-APU & 0.6111111 & 39.26985 & FALSE \\
\hline ACU-ASU & 33.8333333 & 39.26985 & FALSE \\
\hline AE-AES & 4.5555556 & 39.26985 & FALSE \\
\hline AE-AN & 28.8888889 & 39.26985 & FALSE \\
\hline AE-ANP & 37.5000000 & 39.26985 & FALSE \\
\hline AE-ANW & 40.5555556 & 39.26985 & TRUE \\
\hline AE-APU & 33.7222222 & 39.26985 & FALSE \\
\hline AE-ASU & 0.5000000 & 39.26985 & FALSE \\
\hline AES-AN & 24.3333333 & 39.26985 & FALSE \\
\hline AES-ANP & 32.9444444 & 39.26985 & FALSE \\
\hline AES-ANW & 36.0000000 & 39.26985 & FALSE \\
\hline AES-APU & 29.1666667 & 39.26985 & FALSE \\
\hline AES-ASU & 4.0555556 & 39.26985 & FALSE \\
\hline AN-ANP & 8.6111111 & 39.26985 & FALSE \\
\hline AN-ANW & 11.6666667 & 39.26985 & FALSE \\
\hline AN-APU & 4.8333333 & 39.26985 & FALSE \\
\hline AN-ASU & 28.3888889 & 39.26985 & FALSE \\
\hline ANP-ANW & 3.0555556 & 39.26985 & FALSE \\
\hline ANP-APU & 3.7777778 & 39.26985 & FALSE \\
\hline ANP-ASU & 37.0000000 & 39.26985 & FALSE \\
\hline ANW-APU & 6.8333333 & 39.26985 & FALSE \\
\hline ANW-ASU & 40.0555556 & 39.26985 & TRUE \\
\hline APU-ASU & 33.2222222 & 39.26985 & FALSE \\
\hline
\end{tabular}

nula com um nível de significância de 0.05, pois o p-value obtido foi de 0.002742 e, portanto, menor que o nível de significância. Os resultados da análise ANOVA para o custo considerando a quantidade de elementos requeridos são apresentados na Tabela 4.13. A tabela apresenta os n-1 elementos da amostra menos o (DF), a soma de quadrados do fator e a soma de quadrado dos erros (Sum Sq), a média dos quadrados do fator e a média dos quadrados dos erros (Sum Sq), o valor da variância ( $F$ value) e a probabilidade de rejeitar a hipótese $(\operatorname{Pr}>\mathrm{F})$.

Com relação ao custo relacionado ao número de elementos requeridos, o resultado do teste de análise de variância (ANOVA) indica que a hipótese nula deve ser rejeitada e a 
Tabela 4.11: Resultados do teste ANOVA para o custo considerando a quantidade de elementos requeridos.

\begin{tabular}{cccccc}
\hline & Df & Sum Sq & Mean Sq & F value & $\operatorname{Pr}(>\mathrm{F})$ \\
\hline Critérios & 9 & 23.451 & 2.60568 & 3.1483 & 0.002742 \\
Residuals & 78 & 64.556 & 0.82764 & & \\
\hline
\end{tabular}

alternativa aceita, havendo, então, evidências de que existe diferença de custo entre os pares de critérios considerando a quantidade de elementos requeridos. A igualdade entre os níveis é avaliada analisando-se os intervalos de confiança no gráfico de Tukey, apresentado na Figura 4.14. O teste de Tukey realiza a comparação par a par entre todos os níveis de um fator, nesse caso entre todos os diferentes critérios quando o valor "zero" não pertence ao intervalo de confiança para um dado par, pode-se dizer que há diferença entre os pares de critérios. Há, então, evidências de diferença de custo entre os critérios all-nodes (AN) e all-comm-p-uses (ACCU); all-w-nodes (ANW) e all-edges (AE); e all-w-nodes (ANW) e all-nodes (AN).

Outro fator considerado na análise do custo do critério é a quantidade de elementos não-executáveis. O teste de hipóteses para este fator seguiu a mesma estratégia das análises anteriores. Antes da realização do teste estatístico, a fim de validar ou rejeitar a hipótese foi realizado o teste de normalidade da amostra. Foi usado o teste Shapiro-Wilk para a amostra e o resultado obtido foi de p-value $=0.06243$ maior que 0.05 indicando que a suposição de normalidade dos erros não estava sendo violada.

Como a amostra é normal e os dados não são dependentes, o teste estatístico escolhido para o teste de hipóteses foi a análise de variância (ANOVA). O resultado do teste de análise de variância foi de p-value $=0.07903$, conforme apresentado na Tabela 4.12, indicando que a hipótese nula (HN1), que afirma que não há diferença de custo entre os critérios avaliados, não pode ser rejeitada quando consideramos o número de elementos não executáveis. O resultado para a comparação par a par entre os critérios, apresentada no gráfico de Tukey na Figura 4.15 mostra que para todos os pares de critérios analisados a diferença não é significativa como pode ser observado, pois os intervalos de confiança sempre incluem o valor zero.

Com isso, apesar de alguns critérios apresentarem um número maior de elementos não-executáveis, essa diferença não é significativa se a análise do fator custo for feita considerando essa variável. Já em relação ao número de elementos requeridos, alguns critérios apresentaram uma diferença significativa de custo. Assim como para o custo considerando o tamanho do conjunto de casos de teste adequado, há indícios de diferença de custo entre alguns pares distintos de critérios de teste. 


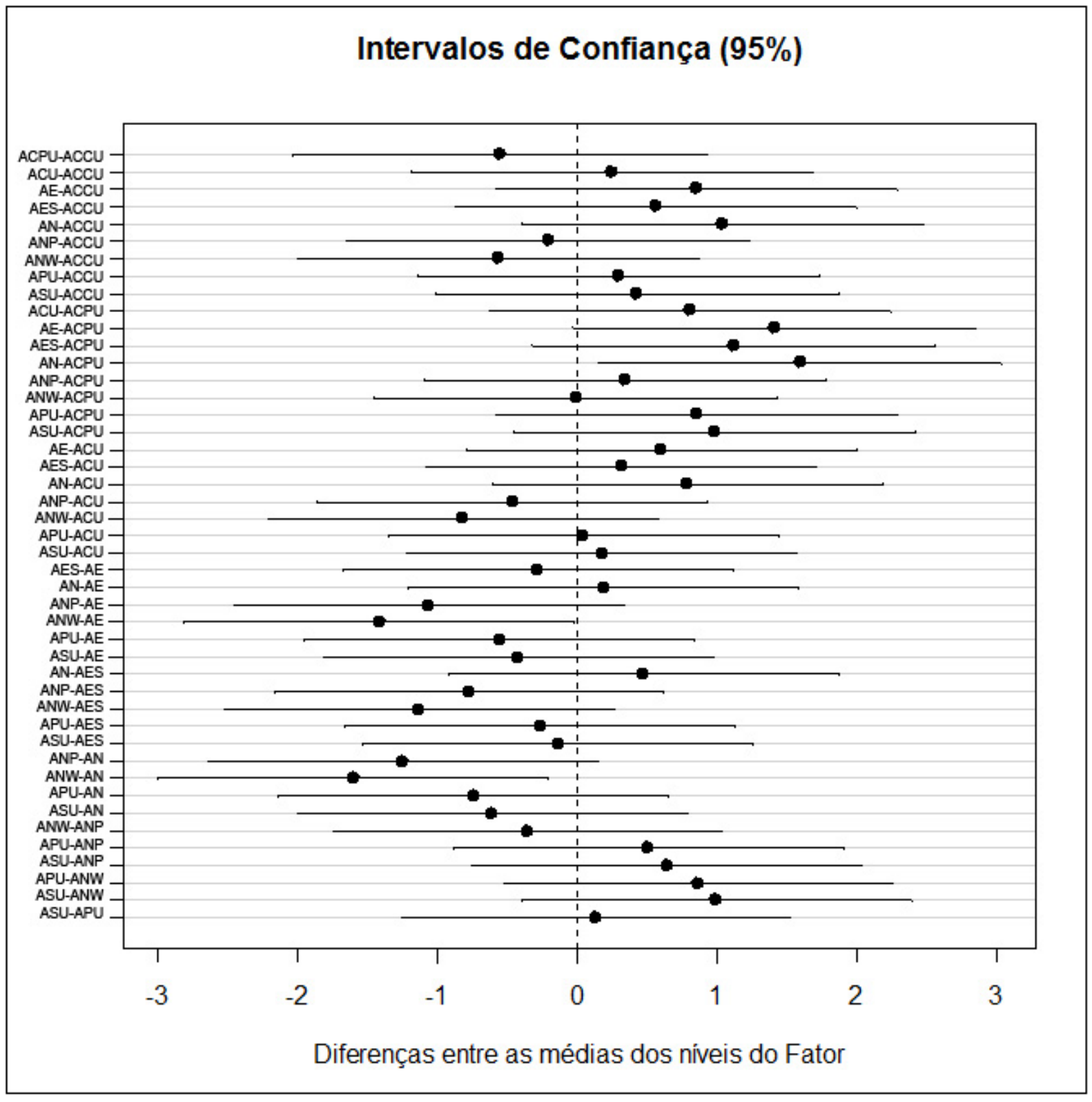

Figura 4.14: Resultados do teste de Tukey para o custo em relação à quantidade de elementos requeridos.

Tabela 4.12: Resultados do teste ANOVA para o custo considerando a quantidade de elementos não-executáveis.

\begin{tabular}{cccccc}
\hline & Df & Sum Sq & Mean Sq & F value & $\operatorname{Pr}(>\mathrm{F})$ \\
\hline Critérios & 9 & 10.154 & 1.12820 & 1.8419 & 0.07903 \\
Residuals & 60 & 36.752 & 0.61253 & & \\
\hline
\end{tabular}

\subsubsection{Eficácia do Critério de Teste}

Para que a hipótese nula correspondente a eficácia (HN2) possa ser rejeitada é necessário que ao menos dois dos dez critérios avaliados apresentem valores distintos de eficácia entre 


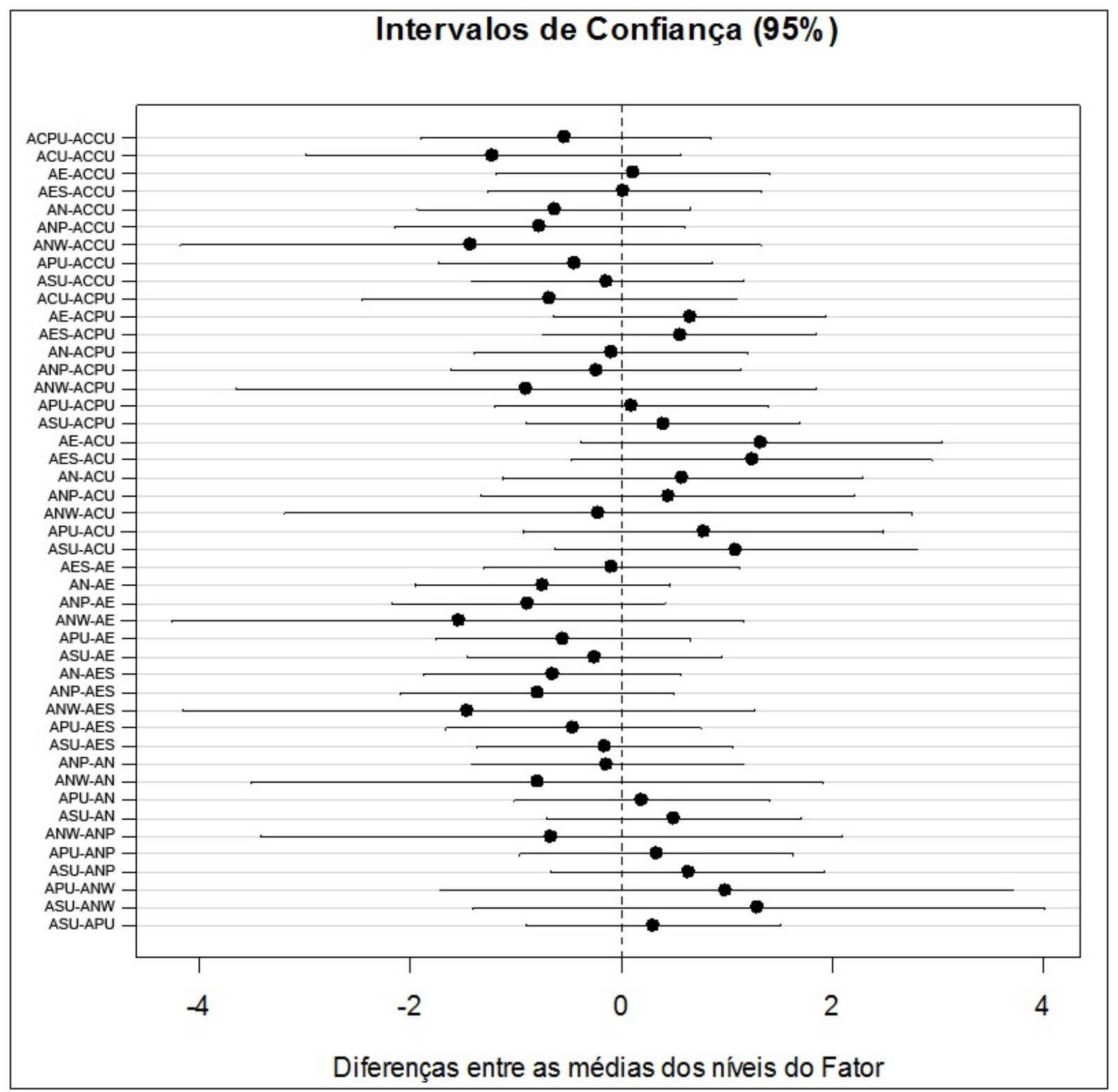

Figura 4.15: Resultados do teste de Tukey para o custo em relação à quantidade de elementos não-executáveis.

si. A eficácia de um critério é determinada com base na porcentagem de defeitos revelados por um critério em relação ao total de defeitos inseridos.

A análise das hipóteses referentes à eficácia dos critérios segue a mesma abordagem utilizada para o teste das hipóteses relacionadas ao custo. A primeira análise indicou que a amostra para a eficácia possui uma distribuição normal com p-value $=0.1765$ portando o uso de testes paramétricos é possível. O teste paramétrico utilizado foi a análise de variância (ANOVA) e os resultados obtidos são apresentados na Tabela 4.13. Com base nesses resultados, a hipótese nula deve ser aceita a hipótese alternativa rejeitada, uma vez que os critérios não apresentaram diferenças significativas de eficácia. 
Tabela 4.13: Resultados do teste ANOVA para a eficácia do critério de teste.

\begin{tabular}{cccccc}
\hline & Df & Sum Sq & Mean Sq & F value & $\operatorname{Pr}(>\mathrm{F})$ \\
\hline Critérios & 9 & 228908 & 25434 & 0.5461 & 0.8364 \\
Residuals & 79 & 3679200 & 46572 & & \\
\hline
\end{tabular}

\subsubsection{Strength}

A análise dos dados relacionados ao strength foi realizada usando o método estatístico de análise de cluster com objetivo de identificar se há relação de inclusão entre os critérios de teste. Gráficos do tipo dendrograma são utilizados para ilustrar os resultados sobre relação de inclusão entre critérios. Nesse gráfico, quando um critério inclui outro critério, ambos devem estar no último nível (nível zero) do gráfico, indicando que a diferença para a cobertura de ambos é nula.

A Figura 4.16 representa o strength para o critério all-nodes (AN). Observa-se que esse critério inclui os critérios all-w-nodes (ANW) e all-p-nodes (ANP), o que já era esperado pois esses critérios são derivados do critério all-nodes.

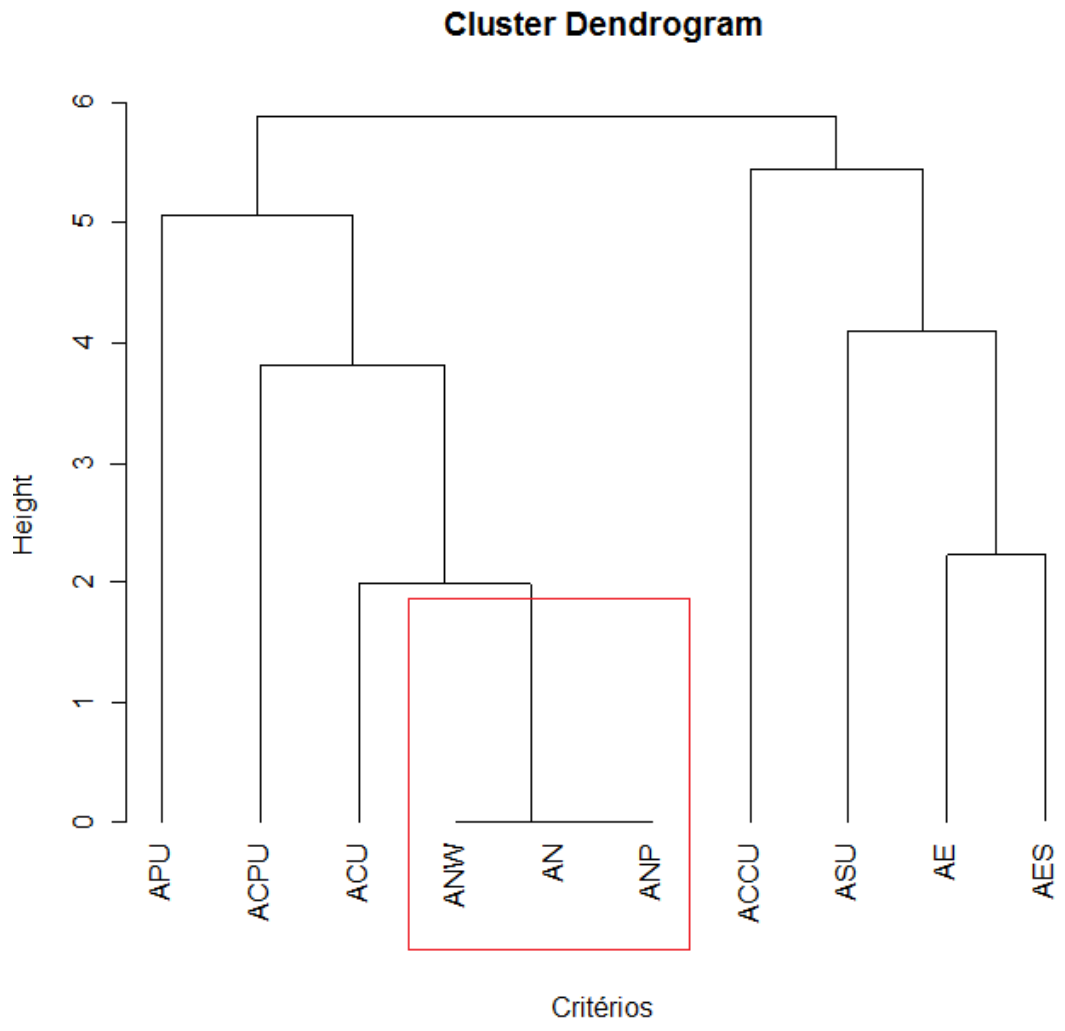

Figura 4.16: Strength para o critério all-nodes (AN).

Os critérios all-w-nodes (ANW) e all-comm-p-uses (ACPU) não apresentam relação de inclusão com nenhum dos demais critérios, conforme pode ser constatado nas Figuras 4.17 e 4.22. Esse resultado é consequência da propriedade do critério all-w-nodes, que é 
bastante restritivo em sua definição, pois inclui apenas nós que contenham a primitiva de sincronização wait.

\section{Cluster Dendrogram}

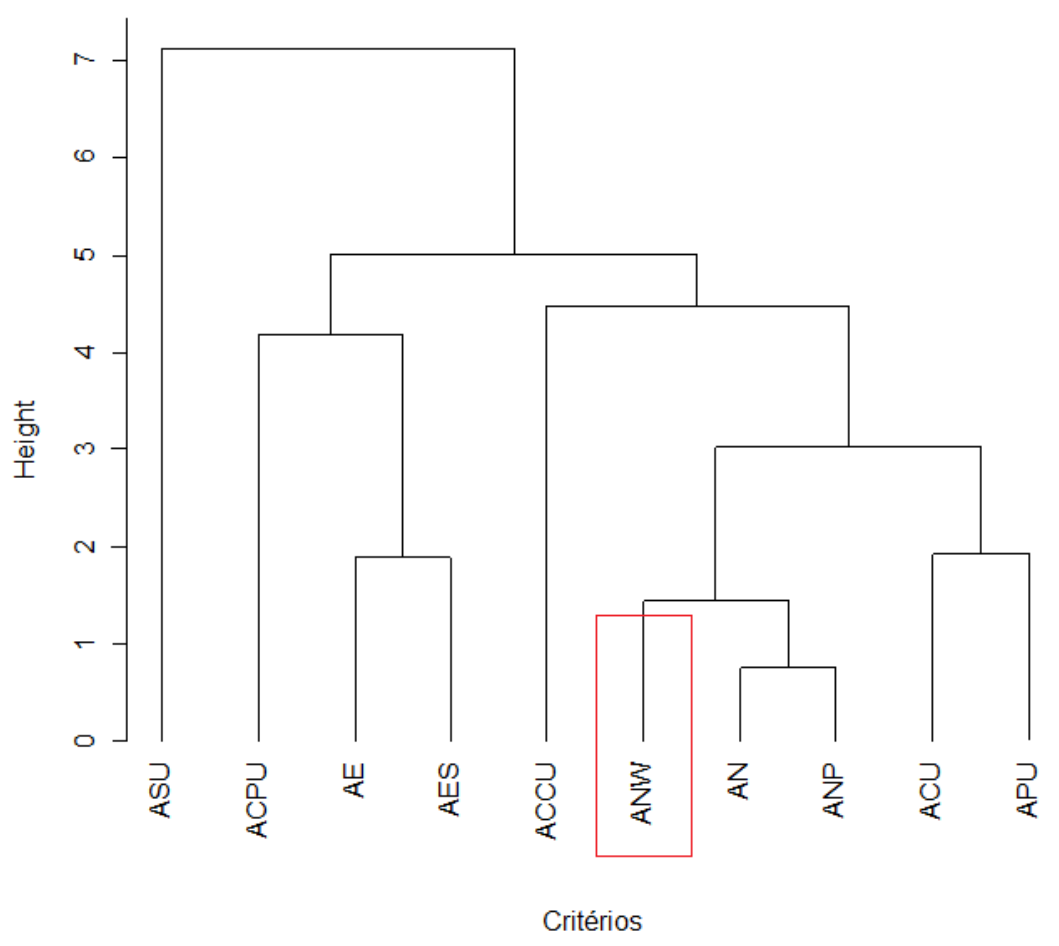

Figura 4.17: Strength para o critério all-w-nodes (ANW).

O dendrograma para o critério all-edges (AE) é ilustrado na Figura 4.18. Para a amostra esse critério inclui os critérios all-s-edges (AES), all-nodes (AN), all-p-nodes (ANW) e all-p-nodes (ANP). Já era esperado que o critério all-edges incluísse o critério all-s-edges que é um subconjunto do critério all-edges. Assim como nos critérios sequenciais, o critério all-edges inclui o critério all-nodes e seus derivados: all-w-nodes e all-p-nodes.

As Figuras 4.19, 4.20 e 4.23 mostram o strength para os critérios all-p-nodes (ANP), all-c-uses (ACU) e all-comm-c-uses (ACCU). Esses critérios incluem somente o critério all-w-nodes (ANW), conforme apresentado. O critério all-w-nodes é pouco custoso e por isso é incluído facilmente por outros critérios. Novas replicações devem ser realizadas a fim de confirmar os resultados obtidos com este experimento em particular.

A Figura 4.21 apresenta o strength para o critério all-p-uses (APU). De acordo com o dendrograma ilustrado na figura, pode-se inferir que o critério all-p-uses inclui o critério all-c-uses (ACU).

O critério all-sync-uses (ASU) inclui os critérios all-w-nodes (ANW) e all-p-nodes (ANP) e all-comm-p-uses (ACPU), conforme o dendrograma apresentado na Figura 4.24. 


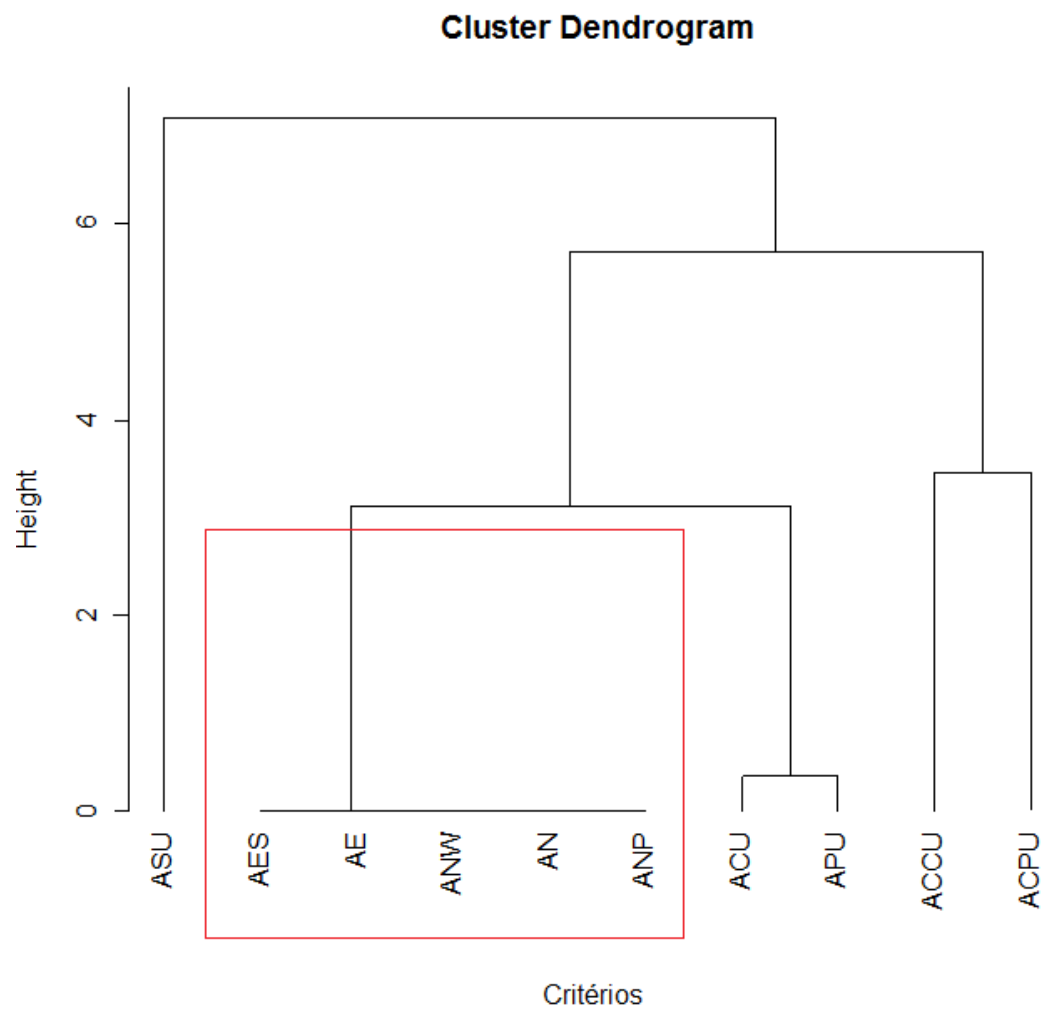

Figura 4.18: Strength para o critério all-edges (AE).

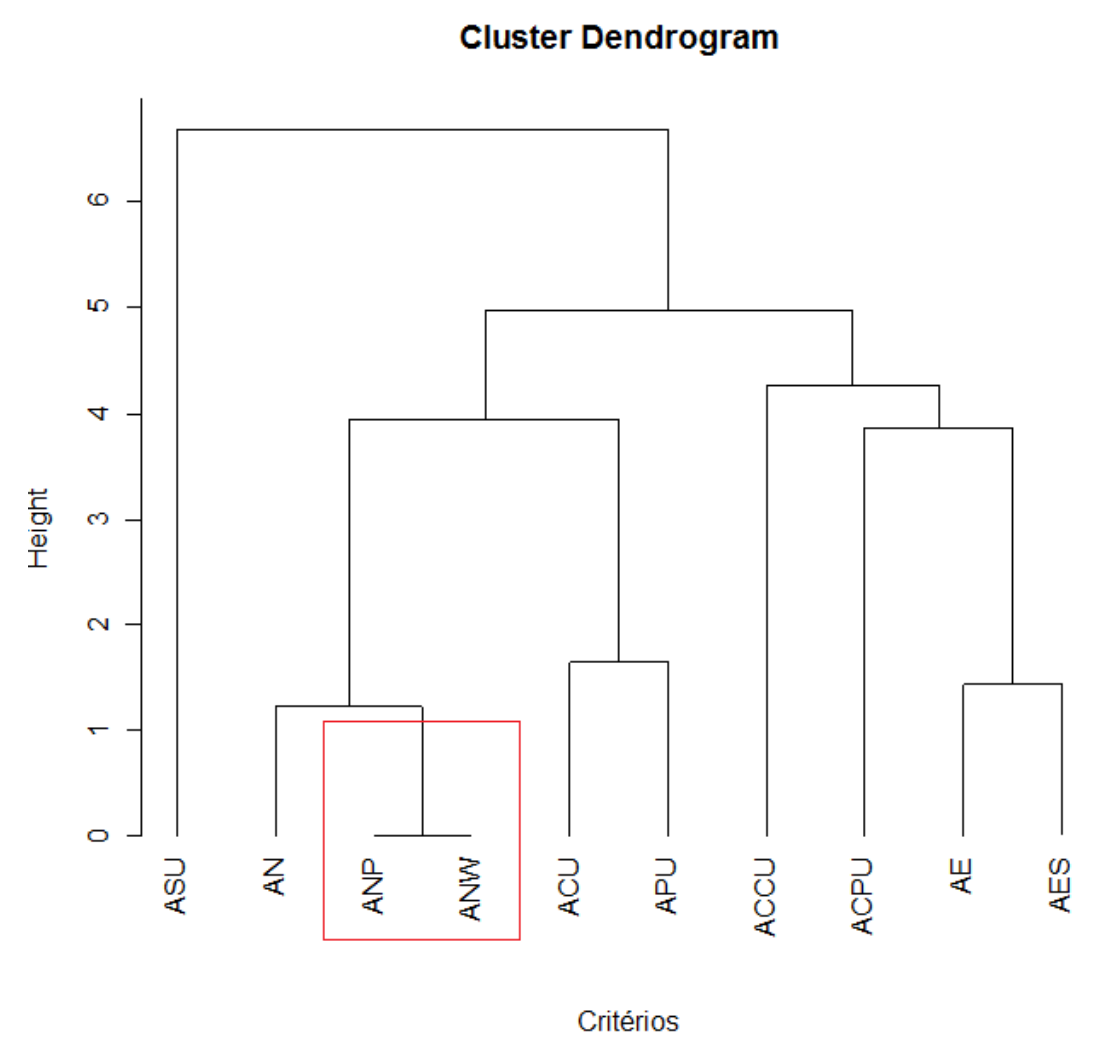

Figura 4.19: Strength para o critério all-p-nodes (ANP). 


\section{Cluster Dendrogram}

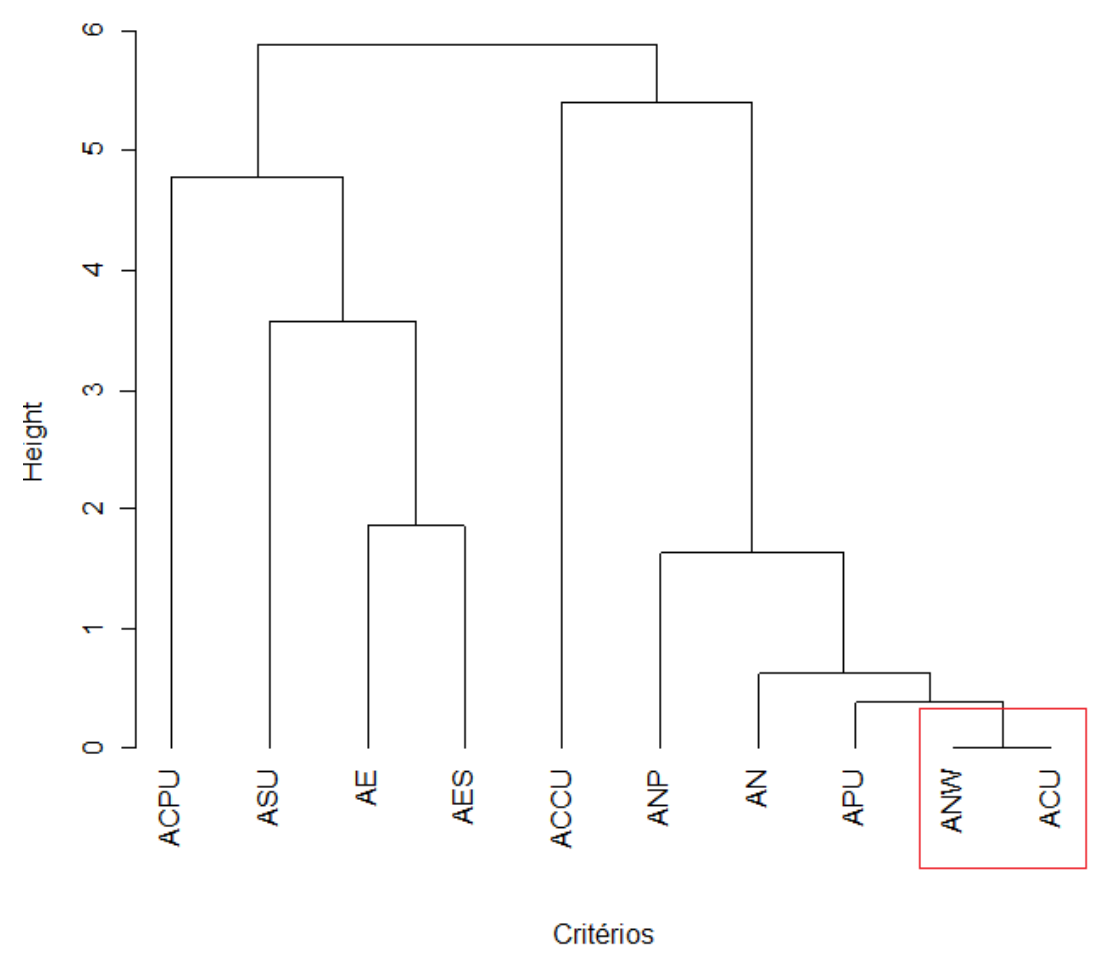

Figura 4.20: Strength para o critério all-c-uses (ACU).

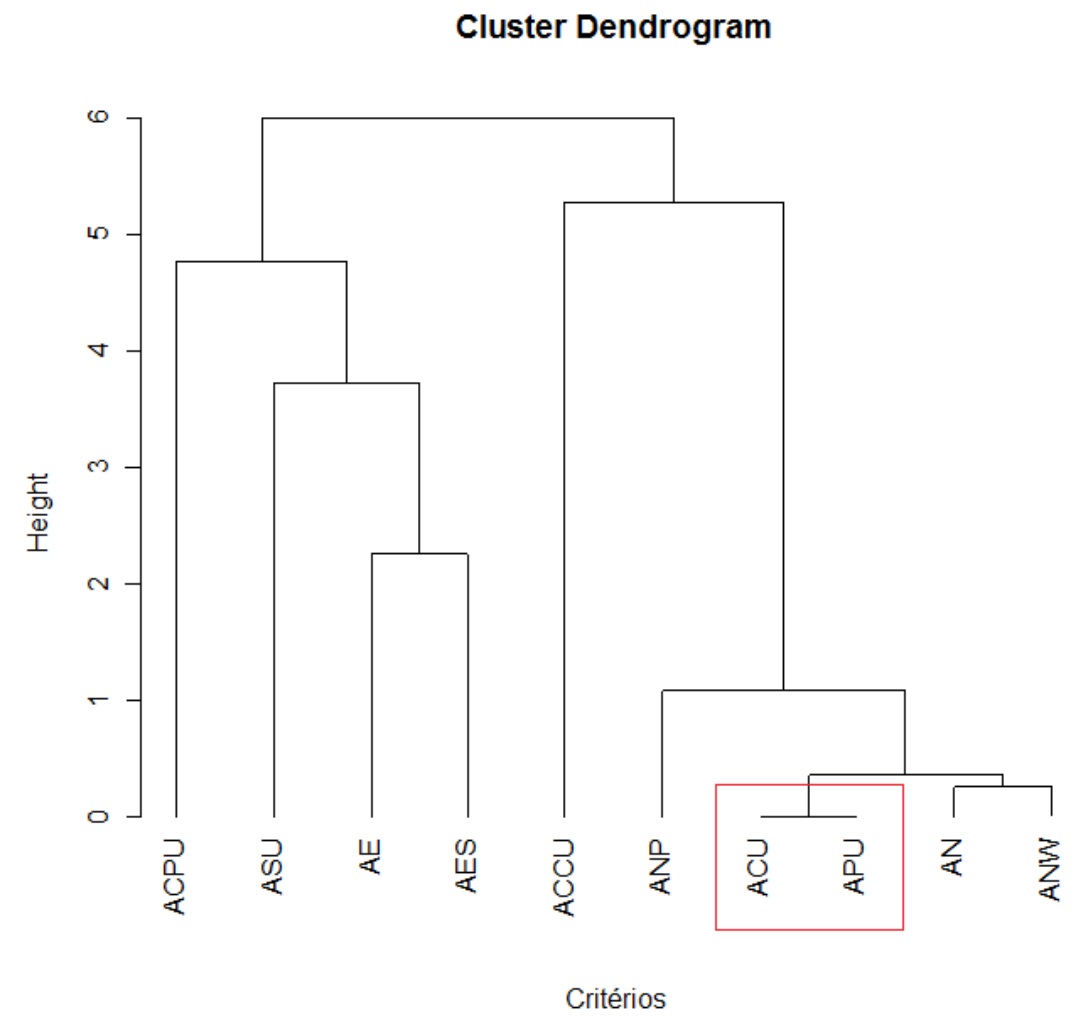

Figura 4.21: Strength para o critério all-p-uses (APU). 


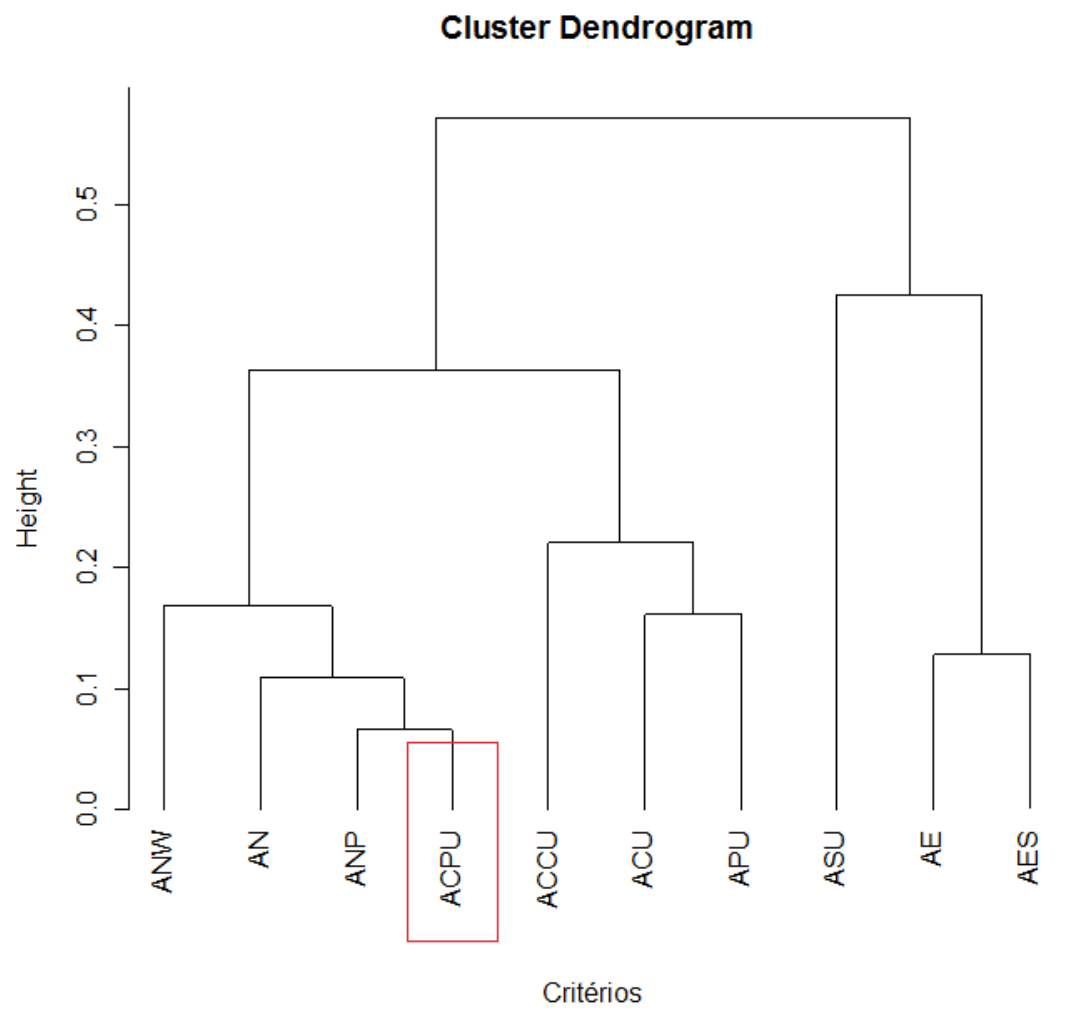

Figura 4.22: Strength para o critério all-comm-p-uses (ACPU).

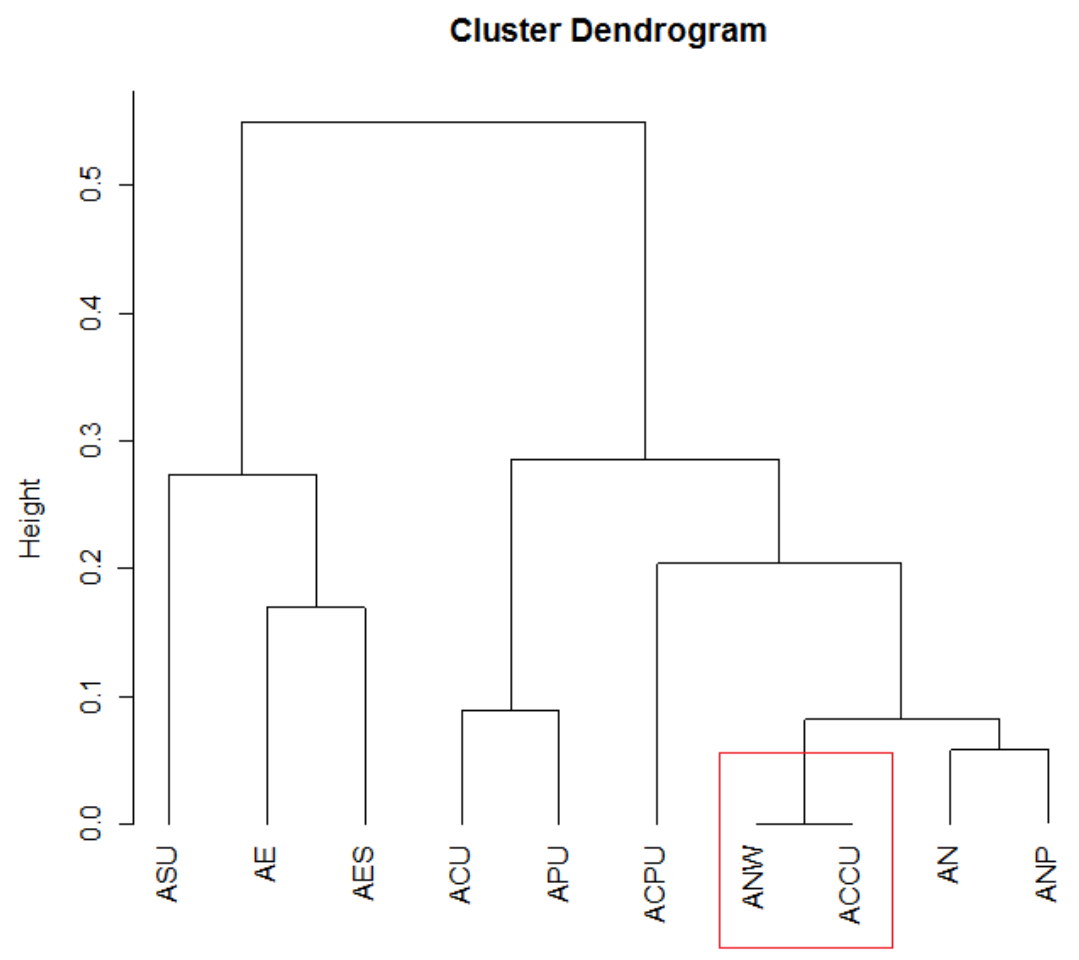

Critérios

Figura 4.23: Strength para o critério all-comm-c-uses (ACCU). 


\section{Cluster Dendrogram}

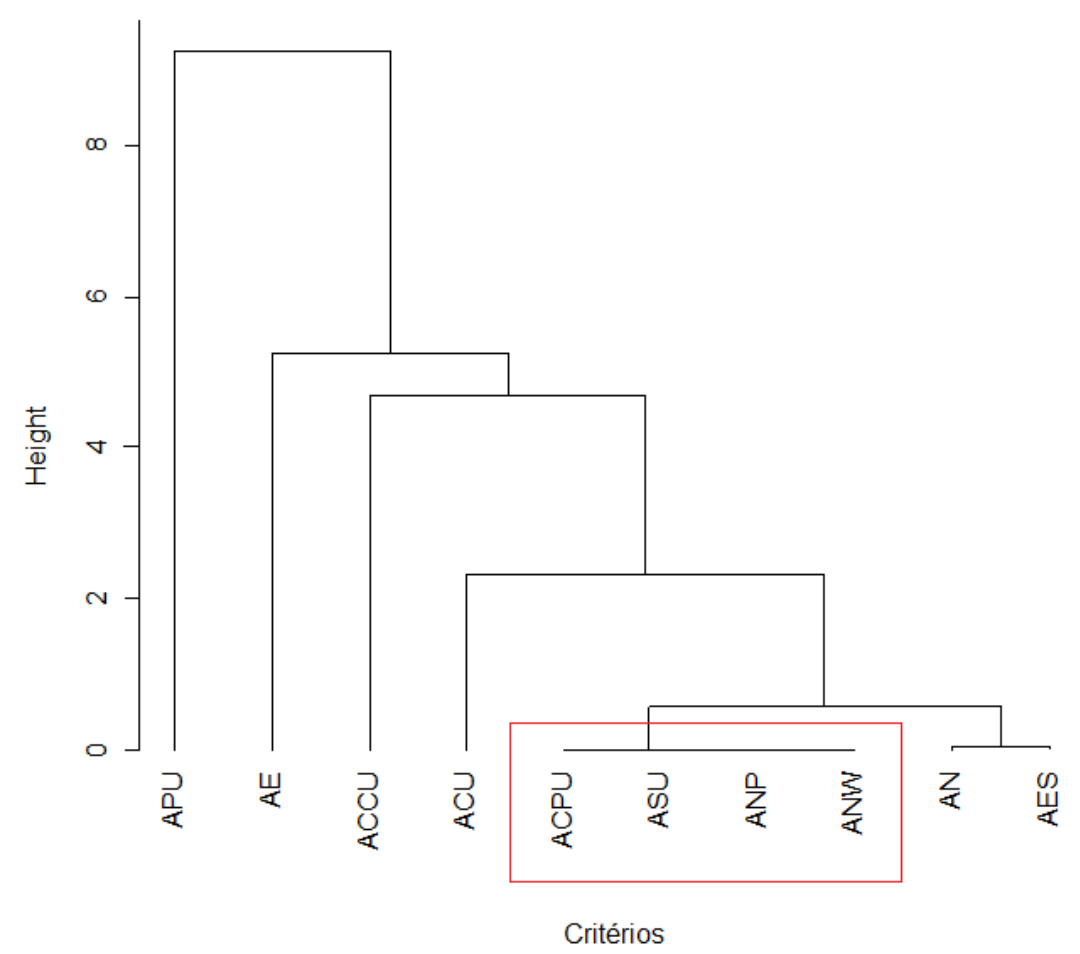

Figura 4.24: Strength para o critério all-sync-uses (ASU).

Analisando a Figura 4.25 pode-se inferir que o critério all-s-edges (AES) inclui o critérios all-w-nodes (ANW) e all-p-nodes (ANP). Assim como acontece nos critérios sequenciais, o critério relacionado a cobertura de arestas inclui os critérios relacionados a cobertura de nós.

\subsubsection{Síntese das Análises}

Os resultados na análise estatística mostraram pequenas diferenças de custo entre os critérios considerando os três fatores estudados. Durante a análise estatística, os critérios foram classificados em ordem crescente de custo, considerando as médias obtidas para cada fator analisado. Essa diferença de custo pode não existir se a diferença estatística não for significativa entre eles, isso é analisado no teste de hipóteses. O teste de hipóteses para esta amostra demonstrou que há diferenças significativas de custo entre os critérios apenas quando é considerado o tamanho do conjunto adequado e a quantidade de elementos requeridos, já quando se considera o número de elementos não-executáveis os critérios apresentam valores muito similares de custo. Assim, ao final do teste de estatístico, os critérios podem ser classificados em ordem decrescente de custo, como segue: all-edges, all-sync-uses, all-s-edges, all-nodes, all-comm-c-uses, all-p-uses, all-c-uses, all-p-nodes, 


\section{Cluster Dendrogram}

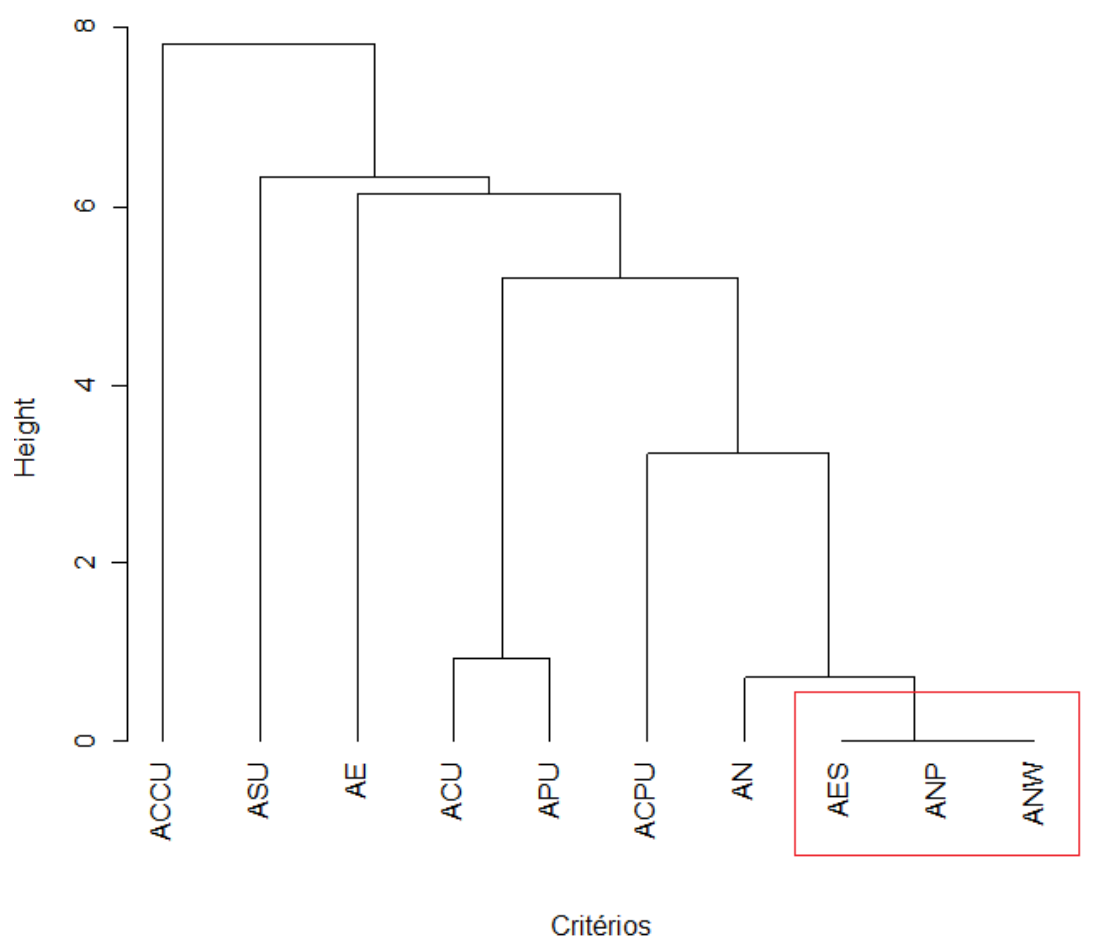

Figura 4.25: Strength para o critério all-s-edges (AES).

all-comm-p-uses e all-w-nodes de acordo com as médias obtidas para cada fator analisado e os resultados do teste de hipóteses.

A análise da eficácia para cada critério mostrou que os critérios não apresentam uma diferença significativa de eficácia em revelar defeitos para a amostra utilizada. Porém, pode-se estabelecer com base na análise descritiva uma classificação, em ordem decrescente de eficácia: all-edges, all-nodes, all-sync-uses, all-s-edges, all-comm-c-uses, all-p-nodes, all-w-nodes, all-c-uses, all-comm-p-uses e all-p-uses. Sendo assim, devido ao fato dos critérios possuirem capacidade similar em revelar defeitos, ficando a escolha do critério a cargo do testador, que pode levar em consideração os demais fatores analisados, como custo e dificuldade de satisfação, a fim de decidir qual critério aplicar durante a atividade de teste.

Quanto ao fator strength, que expressa a dificuldade de satisfação de um critério, os resultados mostram que há evidências de uma relação de inclusão entre os critérios, o que possibilitou estabelecer uma estratégia de aplicação para os critérios. Com base nestes resultados e nos resultados de eficácia e custo, a melhor abordagem para aplicar os critérios deve ser definida pelo testador. Uma possível estratégia de aplicação dos critérios é começar o teste com a aplicação de critérios mais eficazes e em seguida aplicar critérios de menor eficácia a fim de cobrir classes específicas de defeitos. 
Com base na eficácia, uma possível estratégia de aplicação dos critérios de teste poderia ser:

1. Aplica-se o critério all-edges que possui melhor eficácia dentre os demais critérios avaliados. O critério all-edges inclui os critérios all-s-edges, all-nodes, all-p-nodes e all-w-nodes. Assim, a cobertura do critério all-edges proporciona também a cobertura desses critérios.

2. Em seguida, o conjunto de casos de teste adequado ao critério all-edges é aplicado ao critério all-sync-uses, e novos casos de teste são criados a fim de obter uma cobertura total do critério. A cobertura do critério all-sync-uses implica na cobertura do critério all-comm-p-uses e all-w-nodes que são incluídos pelo critério all-sync-uses.

3. Então, novos casos de teste são criados para cobrir os elementos requeridos pelo critério all-comm-c-uses que inclui o critério all-w-nodes.

4. E, por fim, são gerados novos casos de teste para satisfazer o critério all-p-uses que inclui o critério all-c-uses, que por sua vez, inclui o critério all-w-nodes.

Assim, em apenas quatro etapas é provável que se possa satisfazer todos os dez critérios de teste, o que proporciona uma cobertura ampla do programa em teste.

\subsection{Comparação entre Critérios Concorrentes e Sequen- ciais}

Os resultados obtidos anteriormente em relação ao custo e à eficácia, motivaram uma análise comparativa entre os dois grandes grupos nos quais os critérios analisados podem ser divididos critérios de teste sequenciais e critérios definidos para programas concorrentes. Os critérios de teste foram divididos em um grupo composto por critérios utilizados comumente no teste de aplicações sequenciais, fazem parte desse grupo os critérios all-nodes, all-edges, all-c-uses e all-p-uses. O outro grupo é formado por critérios de teste derivados dos critérios de teste sequenciais para tratar características específicas do contexto de programação concorrente, constituem esse grupo os critérios all-p-nodes, all-w-nodes, all-s-edges, all-comm-c-uses, all-comm-p-uses e all-sync-uses.

A análise desses grupos é importante para investigar os benefícios em usar critérios específicos para programas concorrentes na descoberta de defeitos.

\subsubsection{Definição das Hipóteses}

Com base no objetivo proposto, a seguinte questão foi definida: 
Dentre os critérios de teste analisados há diferença de eficácia e custo entre critérios de caráter sequencial quando comparados com os concorrentes?

E buscando responder esta questão as seguintes hipóteses foram formalizadas:

- HN1.1 - Não há diferença significativa de custo entre critérios de teste concorrentes e sequenciais.

- HA1.1 - Os critérios de teste sequenciais apresentam custo maior que os concorrentes.

- HN2.1 - Não há diferença significativa de eficácia entre critérios de teste concorrentes e sequenciais.

- HA2.1 - Os critérios de teste sequenciais apresentam maior eficácia que os concorrentes.

\subsubsection{Teste de Hipóteses}

Como o conjunto de dados é reduzido e a amostra possui menos de 50 elementos, é provável que esta amostra não seja normal e, por isso, durante o teste de hipóteses são usados apenas testes não paramétricos. Devido à distinção dos critérios em dois grupos diferentes, foram utilizados testes estatísticos que possibilitam a análise de fatores por grupos (blocos).

\subsubsection{Análise da Eficácia}

Para avaliação da eficácia entre os dois tipos de critérios, é utilizado o teste de Friedman (Friedman, 1937), o qual é aplicado quando o teste de experimentos envolve blocos. Como os critérios são divididos em dois blocos distintos (sequenciais e concorrentes) e como a amostra é pequena e aparentemente as variações entre as populações é diferente, não se pode garantir a normalidade dos dados. Por esse motivo, optou-se por utilizar este teste não paramétrico que utiliza os ranks dos dados para o cálculo da estatística de teste dos grupos de acordo com os fatores de interesse a serem comparados.

Os resultados da análise geral entre os grupos para o teste de Friedman são apresentados na Tabela 4.15. A tabela apresenta o valor do Qui-quadrado de Friedman, os graus de liberdade (número de grupos -1), e o p-valor para a amostra. Analisando a tabela, pode-se notar que não é possível rejeitar a hipótese nula (HN1.1), que diz que não existe diferença significativa de eficácia entre os critérios, com um nível de significância de 5\%, pois o p-valor apresentado é maior que o intervalo de confiança definido de 0,05.

A Tabela 4.14 apresenta a comparação par a par entre os grupos de critérios sequenciais e concorrentes. Na primeira coluna da tabela são apresentados os fatores (critérios) 
emparelhados, sempre um critério concorrente comparado a um critério sequencial. A segunda coluna traz o valor da diferença média entre os dois grupos, calculada de acordo com o teste de Friedman. Na terceira coluna é apresentado o valor da diferença crítica para a amostra obtido em uma tabela base. Se a diferença entre a média dos dois grupos é maior ou igual a diferença crítica, então há diferença significativa. A última coluna da tabela mostra se existe ou não diferença significativa entre os grupos. Os resultados mostram que para nenhum par de critérios há diferença significativa entre os valores obtidos para eficácia. Ou seja, exitem evidências que a eficácia dos fatores são todas significativamente iguais para esta amostra.

Tabela 4.14: Resultados do teste de Friedman com análise par a par.

\begin{tabular}{|c|c|c|c|}
\hline $\begin{array}{c}\text { Fatores } \\
\text { comparados }\end{array}$ & $\begin{array}{c}\text { Diferença } \\
\text { observada }\end{array}$ & $\begin{array}{c}\text { Diferença } \\
\text { crítica }\end{array}$ & $\begin{array}{c}\text { Diferença } \\
\text { significativa }\end{array}$ \\
\hline ANP-AN & 5,0 & 41,88531677 & Não \\
ANW-AN & 12,0 & 41,88531677 & Não \\
AES-AN & 11,0 & 41,88531677 & Não \\
ASU-AN & 13,5 & 41,88531677 & Não \\
ACCU-AN & 21,5 & 41,88531677 & Não \\
ACPU-AN & 7,5 & 41,88531677 & Não \\
ANP-AE & 20,5 & 41,88531677 & Não \\
ANW-AE & 13,5 & 41,88531677 & Não \\
AES-AE & 14,5 & 41,88531677 & Não \\
ASU-AE & 12,0 & 41,88531677 & Não \\
ACCU-AE & 4,0 & 41,88531677 & Não \\
ACPU-AE & 18,0 & 41,88531677 & Não \\
ANP-ACU & 5,0 & 41,88531677 & Não \\
ANW-ACU & 12,0 & 41,88531677 & Não \\
AES-ACU & 11,0 & 41,88531677 & Não \\
ASU-ACU & 13,5 & 41,88531677 & Não \\
ACCU-ACU & 21,5 & 41,88531677 & Não \\
ACPU-ACU & 7,5 & 41,88531677 & Não \\
ANP-APU & 4,0 & 41,88531677 & Não \\
ANW-APU & 3,0 & 41,88531677 & Não \\
AES-APU & 2,0 & 41,88531677 & Não \\
ASU-APU & 4,5 & 41,88531677 & Não \\
ACCU-APU & 12,5 & 41,88531677 & Não \\
ACPU-APU & 1,5 & 41,88531677 & Não \\
\hline
\end{tabular}

Tabela 4.15: Resultado do teste de Friedman.

\begin{tabular}{cr}
\hline Informação & Valor \\
\hline Friedman qui-quadrado & 11,01895735 \\
Graus de Liberdade & 9 \\
P-valor & 0,274411745 \\
\hline
\end{tabular}

\subsubsection{Análise do Custo}

A análise do custo foi feita utilizando o Teste de Wilcoxon Pareado (Wilcoxon, 1945). A seleção deste teste deve-se ao tipo da comparação que realizada, onde são comparados pares de fatores (critérios) de grupos distintos (sequenciais ou concorrentes) por meio de um teste unilateral para direita (o teste analisa se existem diferenças e se elas são maiores para os critérios concorrentes que para os sequenciais). A hipótese nula (HN2.1) 
é rejeitada se a mediana da diferença não for nula ou se for menor que que o intervalo de confiança, assim, a hipótese alternativa (HA2.1) é aceita se a mediana da diferença não for nula e for maior que o intervalo de confiança estabelecido, neste caso maior que 0,05.

A Tabela 4.16 apresenta os resultados da comparação entre os critérios concorrentes e sequenciais, obtidos utilizando o Teste de Wilcoxon Pareado. Pela análise da tabela, podemos dizer que existem evidências de diferença entre as duas amostras para alguns pares de critérios. Para os pares ANP-AN, ANW-AN, ANP-AE, ANW-AE, AES-AE, ACCU-AE, ACPU-AE e ANP-APU, a diferença não foi significativa. Os critérios concorrentes apresentaram-se mais custosos para os pares AES-AN, ASU-AN, AES-ACU, ASU-ACU, AES-APU e ASU-APU. Para todos os demais pares ACCU-AN, ACPU-AN, ANP-ACU, ANW-ACU, ACCU-ACU, ACPU-ACU, ANW-APU, ACCU-APU e ACPUAPU os critérios concorrentes apresentaram estatisticamente custo menor que os sequenciais.

Tabela 4.16: Resultados do teste de Wilcoxon pareado para o custo considerando o conjunto adequado.

\begin{tabular}{|c|c|c|c|}
\hline Fatores & P-valor & W & Diferença \\
\hline ANP-AN & 0.967200154 & 0.0 & Não \\
ANW-AN & 0.967200154 & 0.0 & Não \\
AES-AN & 0.016407929 & 40.5 & Sim \\
ASU-AN & 0.008877961 & 28.0 & Sim \\
ACCU-AN & 0.375242710 & 12.0 & Sim \\
ACPU-AN & 0.624757290 & 9.0 & Sim \\
ANP-AE & 0.9961838 & 0 & Não \\
ANW-AE & 0.9961838 & 0 & Não \\
AES-AE & 0.9706091 & 0 & Não \\
ASU-AE & 0.7673956 & 3 & Sim \\
ACCU-AE & 0.9910198 & 0 & Não \\
ACPU-AE & 0.9915263 & 0 & Não \\
ANP-ACU & 0.672639577 & 1.0 & Sim \\
ANW-ACU & 0.871580371 & 2.0 & Sim \\
AES-ACU & 0.008980239 & 28.0 & Sim \\
ASU-ACU & 0.003816221 & 45.0 & Sim \\
ACCU-ACU & 0.098733037 & 8.5 & Sim \\
ACPU-ACU & 0.293968373 & 9.5 & Sim \\
ANP-APU & 0.910143753 & 0.0 & Não \\
ANW-APU & 0.929613613 & 1.0 & Sim \\
AES-APU & 0.013923975 & 41.0 & Sim \\
ASU-APU & 0.005756914 & 36.0 & Sim \\
ACCU-APU & 0.298056333 & 13.0 & Sim \\
ACPU-APU & 0.416742002 & 11.5 & Sim \\
\hline
\end{tabular}

Em relação ao número de elementos requeridos, os resultados para o teste de Wilcoxon Pareado apresentados na Tabela 4.17, mostram que para a maioria dos pares de critérios há diferença significativa de custo. Apenas para os pares de critérios ANP-AN, ANW-AN, ANP-AE, ANW-AE, AES-AE, ASU-AE, ANW-ACU o custo não é significativamente diferente. Para os pares AES-AN, ASU-AN, ACCU-AN, ACPU-AN, ACCU-AE, ACPUAE, ANP-ACU, ACCU-ACU, ACPU-ACU, ANP-APU, ANW-APU, AES-APU, ASU-APU, ACCU-APU, ACPU-APU, que representam a maior parte da amostra o custo dos pro- 
gramas concorrentes é menor que o dos sequenciais. Somente para os pares AES-ACU e ASU-ACU o custo dos critérios concorrentes foi significativamente maior.

Tabela 4.17: Resultados do teste de Wilcoxon pareado para o custo considerando o número de elementos requeridos.

\begin{tabular}{|c|c|c|c|}
\hline Fatores & P-valor & W & Diferença \\
\hline ANP-AN & 1.0000000 & 0 & Não \\
ANW-AN & 1.0000000 & 0 & Não \\
AES-AN & 0.9628906 & 8 & Sim \\
ASU-AN & 0.9628906 & 8 & Sim \\
ACCU-AN & 0.8085938 & 12 & Sim \\
ACPU-AN & 0.9843750 & 2 & Sim \\
ANP-AE & 1.0000000 & 0 & Não \\
ANW-AE & 1.0000000 & 0 & Não \\
AES-AE & 0.9961838 & 0 & Não \\
ASU-AE & 0.9961838 & 0 & Não \\
ACCU-AE & 0.9537543 & 6 & Sim \\
ACPU-AE & 0.9453125 & 5 & Sim \\
ANP-ACU & 0.99414062 & 3.0 & Sim \\
ANW-ACU & 0.99424309 & 0.0 & Não \\
AES-ACU & 0.02490183 & 39.0 & Sim \\
ASU-ACU & 0.03303898 & 38.0 & Sim \\
ACCU-ACU & 0.58338419 & 16.5 & Sim \\
ACPU-ACU & 0.71093750 & 11.0 & Sim \\
ANP-APU & 0.9863281 & 5.0 & Sim \\
ANW-APU & 0.9896469 & 1.5 & Sim \\
AES-APU & 0.1313091 & 26.0 & Sim \\
ASU-APU & 0.2851562 & 28.0 & Sim \\
ACCU-APU & 0.5781250 & 17.0 & Sim \\
ACPU-APU & 0.6999103 & 8.0 & Sim \\
\hline
\end{tabular}

O teste de hipóteses para o custo dos critérios em relação à quantidade de elementos não executáveis também seguiu o teste de Wilcoxon Pareado e os resultados são mostrados na Tabela 4.18. Considerando os dados da tabela podemos dizer que existem evidências de diferença entre os dois grupos de critérios para os pares ANP-AN, ASU-AN, ACCU-AN, ACPU-AN, ASU-AE, ACCU-AE, ACPU-AE, ANP-ACU, ANW-ACU, ANP-APU, ASUAPU, ACCU-APU, ACPU-APU, onde o p-valor é sempre maior que 0,05, provando que estatisticamente, com um intervalo de confiança de $95 \%$ o custo dos critérios concorrentes é menor que o custo dos sequenciais. Já para os pares ANW-AN, ANP-AE, ANW-AE, AES-AE e ANW-APU a diferença de custo não é significante. E para os pares de critérios AES-AN, AES-ACU, ASU-ACU, ACCU-ACU, ACPU-ACU e AES-APU o custo dos concorrentes foi maior que o dos sequenciais.

\subsubsection{Síntese das Análises}

A Tabela 4.19 apresenta os resultados do teste de hipótese para cada uma das hipóteses definidas nas seções anteriores. Os resultados mostram que de maneira geral os critérios apresentam diferença significativa de custo, sendo que os critérios concorrentes tendem a ser menos custosos que os sequenciais. Em relação à eficácia os critérios apresentaram resultados bastante similares e, portanto nenhuma diferença significativa é encontrada. 
Tabela 4.18: Resultados do teste de Wilcoxon pareado para o custo considerando a quantidade de elementos não executáveis.

\begin{tabular}{|c|c|c|c|}
\hline Fatores & P-valor & W & Diferença \\
\hline ANP-AN & 0.919799595 & 8.0 & Sim \\
ANW-AN & 0.994395133 & 0.0 & Não \\
AES-AN & 0.005396391 & 44.0 & Sim \\
ASU-AN & 0.013923975 & 41.0 & Sim \\
ACCU-AN & 0.070497041 & 28.5 & Sim \\
ACPU-AN & 0.135520640 & 20.5 & Sim \\
ANP-AE & 0.9962632 & 0.0 & Não \\
ANW-AE & 0.9962895 & 0.0 & Não \\
AES-AE & 0.9865717 & 0.0 & Não \\
ASU-AE & 0.9227967 & 10.5 & Sim \\
ACCU-AE & 0.8368021 & 11.0 & Sim \\
ACPU-AE & 0.6234238 & 9.0 & Sim \\
ANP-ACU & 0.084102070 & 17 & Sim \\
ANW-ACU & 0.857475296 & 1 & Sim \\
AES-ACU & 0.005808022 & 36 & Sim \\
ASU-ACU & 0.005859343 & 36 & Sim \\
ACCU-ACU & 0.007979402 & 28 & Sim \\
ACPU-ACU & 0.039062500 & 25 & Sim \\
ANP-APU & 0.91365311 & 11 & Sim \\
ANW-APU & 0.99618378 & 0 & Não \\
AES-APU & 0.01392398 & 41 & Sim \\
ASU-APU & 0.10636004 & 33 & Sim \\
ACCU-APU & 0.28746704 & 22 & Sim \\
ACPU-APU & 0.23153551 & 14 & Sim \\
\hline
\end{tabular}

Além disso, em relação ao fator strength o teste de análise de cluster mostrou a possibilidade de existência de relações empíricas de inclusão entre os critérios. Os resultados detalhados para cada fator são apresentados a seguir.

Tabela 4.19: Resultados do teste de hipóteses.

\begin{tabular}{|l|l|l|}
\hline Hipótese & Definição & Resultado \\
\hline HN1 & Não há diferença de custo entre os critérios & rejeitada \\
\hline HA1 & Há diferença de custo entre os critérios & aceita \\
\hline HN1.1 & Não há diferença de custo entre critérios sequenciais e concorrentes & rejeitada \\
\hline HA1.1 & Há diferença de custo entre critérios sequenciais e concorrentes & aceita \\
\hline HN2 & Não há diferença de eficácia entre os critérios & aceita \\
\hline HA2 & Há diferença de eficácia entre os critérios & rejeitada \\
\hline HN2.1 & Não há diferença de eficácia entre critérios sequenciais e concorrentes & aceita \\
\hline HA2.1 & Há diferença de eficácia entre critérios sequenciais e concorrentes & rejeitada \\
\hline HN3 & Não há relação de inclusão entre os critérios & rejeitada \\
\hline HA3 & Há relação de inclusão entre os critérios & aceita \\
\hline
\end{tabular}

Ao comparar os critérios de teste utilizados no contexto da programação sequencial com critérios derivados para programas concorrentes, foram obtidas evidências estatísticas que os critérios concorrentes são equivalentes em termos de eficácia em detectar defeito não apresentando diferenças significativas para este fator, quando comparados aos sequenciais, considerando os tipos de defeitos e programas avaliados nesse estudo, com um intervalo de confiança em torno de $95 \%$.

Os resultados para o custo de aplicação dos critérios revelaram diferenças significativas entre pares de critérios sequenciais e concorrentes. De maneira geral, quando é considerado o custo em relação ao número de elementos não executáveis os critérios concorrentes all-s-edges (AES), all-sync-uses (ASU), all-comm-c-uses (ACCU) e all-comm-p-uses 
(ACPU) apresentam custo significativamente maior quando comparados a determinados critérios sequenciais. Em relação ao tamanho do conjunto adequado e o número de elementos requeridos, apenas os critérios concorrentes all-s-edges (AES) e all-sync-uses (ASU) apresentam custo de aplicação maior que os sequenciais. O que indica que, em média, o custo dos critérios concorrentes é menor ou igual ao custo de aplicação despendido para satisfazer os critérios sequenciais com uma eficácia equivalente. Esses resultados encorajam o uso de critérios de teste estrutural definidos para programas concorrentes com memória compartilhada a fim de diminuir o esforço, sem perder a qualidade, da atividade de teste.

\subsection{Apresentação e Empacotamento}

Após a realização do experimento é importante que os resultados obtidos sejam apresentados. A apresentação, pode ser feita por meio de artigos, relatórios técnicos, ou material didático (Wohlin et al., 2000). Os resultados parciais deste estudo foram reunidos em um artigo intitulado "Structural Testing for Multithreaded Programs: An Experimental Evaluation of the Cost, Strength and Effectiveness" (Melo et al., 2012), apresentado na 24th International Conference on Software Engineering and Knowledge Engineering, o artigo foi publicado e encontra-se disponível nos proceedings da conferência. Os demais resultados são apresentados nesta dissertação e em um pacote de laboratório de acesso público.

Em um repositório de dados na forma de pacote de laboratório pode ser encontrado todo material utilizado durante a condução deste experimento. São disponibilizados os programas selecionados dos benchmarks, os programas com defeitos inseridos, os conjuntos de casos de testes definidos, as tabelas com os resultados da avaliação dos fatores analisados no estudo, custo eficácia e strength. Além disso, a ferramenta ValiPThread é disponibilizada para download e instalação.

O acesso a este material é importante para facilitar futuras replicações do estudo e comparação da técnica avaliada nesse estudo com as demais técnicas propostas para o teste no contexto da programação concorrente. Além disso, oferece material que pode auxilar o ensino e treinamento de teste de aplicações concorrentes na academia. O uso de um repositório virtual facilita a recuperação dos dados.

O pacote de laboratório para este experimento pode ser obtido no seguinte endereço: http://stoa.usp.br/silvanamm/files. 


\subsection{Considerações Finais}

Ao final das análises apresentadas nesse capítulo é possível responder a questão de pesquisa definida no início do estudo experimental que buscava saber qual a eficácia em descobrir defeitos, o custo de aplicação e o strength dos critérios de teste estrutural definidos para programas concorrentes. Uma estratégia de aplicação desses critérios foi definida com base na análise desses fatores. Conclusões finais sobre o estudo realizado são apresentadas no próximo capítulo. 



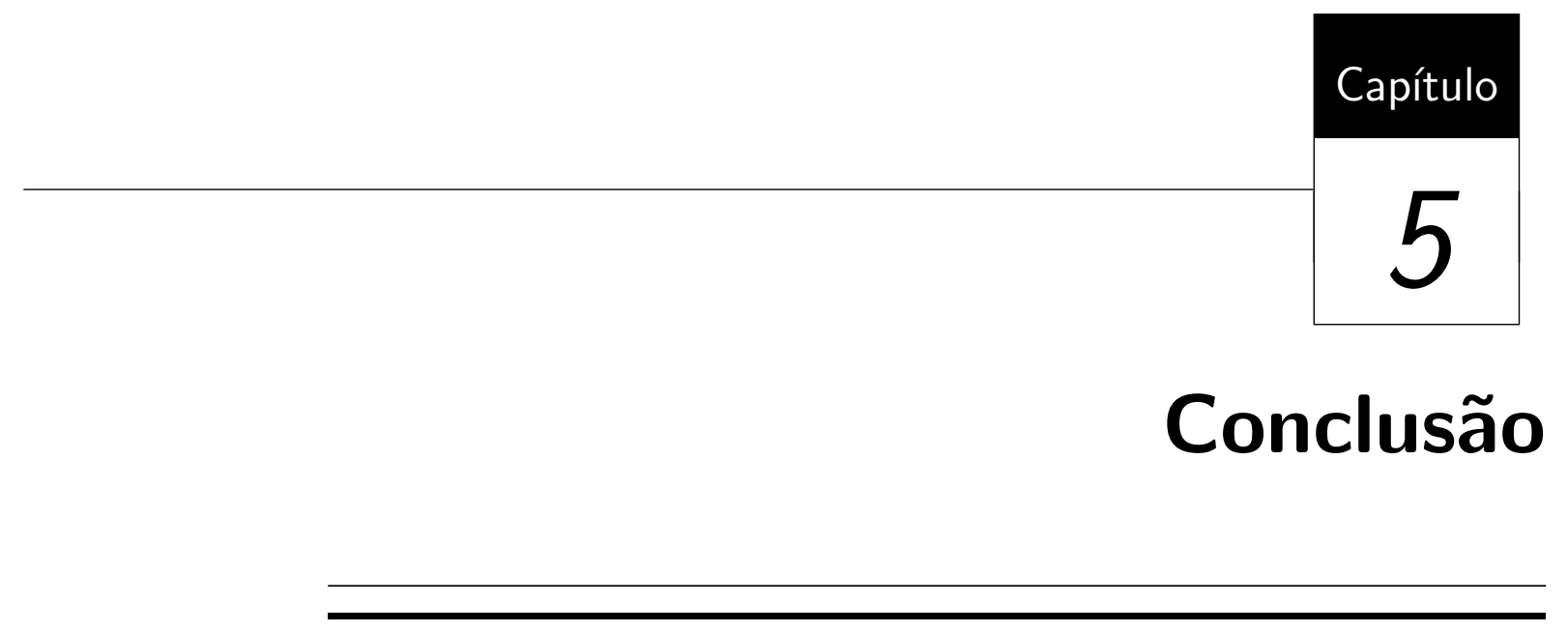

\subsection{Caracterização da Contribuição}

Este projeto de mestrado teve como objetivo estabelecer e aplicar um estudo experimental para avaliar o custo de aplicação a eficácia em detectar defeitos e o aspecto complementar dos critérios de teste definidos para programas concorrentes no contexto de memória compartilhada. Para conduzir esse experimento foram utilizados programas provenientes de benchmarks amplamente utilizados por pesquisadores para avaliação de técnicas de teste para programas concorrentes, o que facilita a comparação da técnica avaliada com as demais técnicas de teste existentes. Esse trabalho complementa o trabalho de Sarmanho (Sarmanho et al., 2008), que define os critérios e a ferramenta de teste ValiPThread, porém não avalia sua aplicabilidade por meio de estudos teóricos ou empíricos. Com base nos resultados obtidos com a utilização da estatística descritiva neste experimento, foi possível classificar os critérios quanto ao custo despendido para satisfazê-los e a sua eficácia em revelar defeitos. Para o fator strength por meio do teste de hipóteses foi possível estabelecer relações entre os critérios. Uma estratégia de aplicação dos critérios foi definida, considerando aspectos de eficácia e custo. Uma análise comparativa entre os critérios de caráter sequencial e critérios concorrentes foi realizada, buscando investigar quais os benefícios do uso dos critérios de teste definidos para programas concorrentes com memória compartilhada, em contrapartida aos critérios de caráter sequencial. Essa análise indicou que os critérios concorrentes possuem um custo menor e eficácia similar 
aos critérios sequenciais, demonstrando que esses critérios são relevantes. Vale ressaltar que em relação à amostra utilizada nesse experimento há evidências que alguns tipos de defeitos, por exemplo deadlock e bloqueio de região crítica, são em alguns casos revelados apenas por critérios concorrentes. Os resultados obtidos foram reunidos em um pacote de laboratório que está disponível para acesso público. Esse pacote de laboratório pode ser utilizado em novas replicações do experimento a fim de comprovar os resultados obtidos, bem como avaliar a estratégia de teste proposta.

\subsection{Contribuições da Pesquisa}

Podem-se destacar como principais contribuições deste trabalho:

- Os resultados obtidos a respeito da comparação do custo para os critérios analisados.

- Os resultados a respeito da eficácia em detectar defeitos dos critérios.

- Os resultados a respeito do strength dos critérios e o estabelecimento de uma relação empírica entre os critérios.

- Avaliação da aplicabilidade do modelo e os critérios de teste propostos no trabalho de Sarmanho et al. (2008).

- Definição de uma estratégia de aplicação dos critérios de teste para programas concorrentes. A estratégia leva em consideração aspectos de custo e eficácia analisados no estudo experimental.

- Realização de uma análise comparativa entre critérios de caráter sequencial e critérios concorrentes. Ressaltando as contribuições dos critérios concorrentes à atividade de teste.

- Disponibilização de um pacote de laboratório que pode ser usado para ensinar conceitos sobre teste de programas concorrentes, facilitar a comparação destes critérios com outras técnicas de teste, e também avaliar outras ferramentas de teste. Enfim, oferece suporte a novos estudos experimentais e replicações deste experimento. $\mathrm{O}$ pacote pode ser acessado nesse endereço: http://stoa.usp.br/silvanamm/files.

\subsection{Trabalhos Futuros}

A replicação deste estudo em trabalhos futuros é importante, a fim de comprovar os resultados obtidos. Alguns resultados dão indícios que tipos distintos de defeitos são 
descobertos em alguns casos apenas por critérios concorrentes, esse fato deve ser analisado com mais rigor em estudos futuros. A replicação em outros ambientes com programas reais seria interessante para estender os resultados da pesquisa do contexto acadêmico para o industrial. A comparação do uso dos critérios de teste estrutural com outras técnicas de teste no contexto de programas concorrentes com memória compartilhada seria de grande valia, a fim de verificar qual a aplicabilidade real da técnica analisada nesse estudo em detrimento das demais. Esses entre outros estudos seriam interessantes para o trabalho desenvolvido e podem vir a fazer parte de trabalhos futuros do grupo de pesquisa. 



\section{Referências}

Almasi, G. S.; Gottlieb, A. Highly parallel computing. Redwood City, CA, USA: Benjamin-Cummings Publishing Co., Inc., 1989.

Andrews, G. R. Concurrent programming: principles and practice. Redwood City, CA, USA: Benjamin-Cummings Publishing Co., Inc., 1991.

Beizer, B. Software testing techniques. 2 ed. New York, NY, USA: Van Nostrand Reinhold Co., 1990.

Binder, R. V. Testing object-oriented systems: models, patterns, and tools. Boston, MA, USA: Addison-Wesley Longman Publishing Co., Inc., 1999.

Bradbury, J. S.; Jalbert, K. Defining a catalog of programming anti-patterns for concurrent java. In: Proc. of SPAQu'09, 2009, p. 6-11.

Brito, M. A. S. Avaliação da efetividade dos critérios de teste estruturais no contexto de programas concorrentes. Dissertação de Mestrado, Instituto de Ciências Matemáticas e de Computação da Universidade de São Paulo, ICMC/USP, São Carlos, SP, 2011.

Camillo, M. S. Avaliação experimental do teste temporal na atividade de teste de programas paralelos. Trabalho de Conclusão de Curso, ICMC/USP, São Carlos, 2008.

Cordeiro, L. Smt-based bounded model checking for multi-threaded software in embedded systems. In: Proceedings of the 32nd ACM/IEEE International Conference on Software Engineering - Volume 2, ICSE '10, New York, NY, USA: ACM, 2010, p. 373-376 (ICSE ' 10, ).

Cordeiro, L.; Fischer, B. Verifying multi-threaded software using smt-based context-bounded model checking. In: Proceedings of the 33rd International Conference on Software Engineering, ICSE '11, New York, NY, USA, 2011, p. 331-340. 
Coward, P. D. A review of software testing. Inf. Softw. Technol., v. 30, p. 189-198, 1988.

Delamaro, M. E.; Maldonado, J. C.; Jino, M. Introdução ao teste de software, v. 394 de Campus. Elsevier, 2007.

DeMillo, R. A.; Lipton, R. J.; Sayward, F. G. Hints on test data selection: Help for the practicing programmer. Computer, v. 11, n. 4, p. 34-41, 1978.

Deniz, E.; Sen, A.; Holt, J. Verification and coverage of message passing multicore applications. ACM Trans. Des. Autom. Electron. Syst., v. 17, n. 3, p. 23:1-23:31, 2012.

Dijkstra, E. W. Cooperating sequential processes, Technical Report, EWD-123. 1965.

Dongarra, J.; Foster, I.; Fox, G.; Gropp, W.; Kennedy, K.; Torczon, L.; White, A., eds. Sourcebook of parallel computing. San Francisco, CA, USA: Morgan Kaufmann Publishers Inc., 2003.

Edelstein, O.; Farchi, E.; Goldin, E.; Nir, Y.; Ratsaby, G.; Ur, S. Framework for testing multi-threaded java programs. Concurrency and Computation: Practice and Experience, v. 15, n. 3-5, p. 485-499, 2003.

Endo, A. T.; Simão, A. S.; Souza, S. R. S. S.; Souza, P. S. L. Web services composition testing: A strategy based on structural testing of parallel programs. Practice And Research Techniques, Testing: Academic \& Industrial Conference on, v. 0, p. 3-12, 2008 .

Frankl, P. G. The use of data flow information for the selection and evaluation of software test data. Tese de Doutoramento, New York University, New York, NY, USA, 1987.

Friedman, M. The use of ranks to avoid the assumption of normality implicit in the analysis of variance. Journal of the American Statistical Association, v. 32, n. 200, p. 675-701, 1937.

Grama, A.; Karypis, G.; Kumar, V.; Gupta, A. Introduction to Parallel Computing. 2 ed. Addison Wesley, 2003.

Hansen, P. B. Operating system principles. Upper Saddle River, NJ, USA: Prentice-Hall, Inc., 1973.

Hausen, A. C.; Vergilio, S. R. Valimpi: Uma ferramenta de teste estrutural para programas paralelos em ambiente de passagem de mensagem. Dissertação de Mestrado, 
Universidade Federal do Paraná, Setor de Ciências Exatas, Programa de Pós-Graduação em Informática, Curitiba, PR, 2005.

Hausen, A. C.; Vergilio, S. R.; Souza, S. R. S.; Souza, P. S. L.; Simão, A. A tool for structural testing of mpi programs. in: Latin-american test workshop - latw. In: 8th IEEE LAtin-American Test Workshop, Cuzco, Peru, 2007, p. 1-1.

Herman, P. M. A data flow analysis approach to program testing. Australian Computer Journal, v. 8, n. 3, p. 92-96, 1976.

Hoare, C. A. R. Monitors: an operating system structuring concept. Commun. ACM, v. 17, p. 549-557, 1974.

Howden, W. E. Reliability of the path analysis testing strategy. IEEE Trans. Softw. Eng., v. 2, p. 208-215, 1976.

Joshi, P.; Naik, M.; Park, C.; Sen, K. Calfuzzer: An extensible active testing framework for concurrent programs. In: Proceedings of the 21st International Conference on Computer Aided Verification, CAV '09, Berlin, Heidelberg: Springer-Verlag, 2009, p. $675-681$.

Koppol, P. V.; Carver, R. H.; Tai, K.-C. Incremental integration testing of concurrent programs. IEEE Trans. Softw. Eng., v. 28, p. 607-623, 2002.

Laski, J. W.; Korel, B. A data flow oriented program testing strategy. IEEE Trans. Softw. Eng., v. 9, p. 347-354, 1983.

Lastovetsky, A. L. Parallel computing on heterogeneous networks. 1 ed. New York, NY, USA: John Wiley \& Sons, Inc., 2003.

Lei, Y.; Carver, R. H. Reachability testing of concurrent programs. IEEE Transactions on Software Engineering, v. 32, p. 382-403, 2006.

Machado, M. C. C. Avaliando a eficácia de critérios de teste para programas concorrentes. Dissertação de Mestrado, Instituto de Ciências Matemáticas e de Computação da Universidade de São Paulo, ICMC-USP, São Carlos, SP, 2008.

Maldonado, J. C. Critérios Potenciais Usos: Uma Contribuição ao Teste Estrutural de Software. Tese de Doutoramento, DCA/FEEC/UNICAMP, Campinas, SP, 1991.

Mamun, M. A. A.; Khanam, A.; Grahn, H.; Feldt, R. Comparing four static analysis tools for java concurrency bugs. In: Proc. of the Third Swedish Workshop on Multi-Core Computing (MCC-10), 2010, p. 143-146. 
Mathur, A. P.; Wong, E. W. An empirical comparison of mutation and data flow based test adequacy criteria. Journal of Software Testing, Verification, and Reliability 4(1): 9-31, 1993.

McCabe, T. A complexity measure. IEEE Transactions on Software Engineering, v. 2, p. 308-320, 1976.

Melo, S. M.; do Rocio Senger de Souza, S.; de Souza, P. S. L. Structural testing for multithreaded programs: An experimental evaluation of the cost, strength and effectiveness. In: SEKE, 2012, p. 476-479.

Mühlenfeld, A.; Wotawa, F. Fault detection in multi-threaded c++ server applications. Electron. Notes Theor. Comput. Sci., v. 174, n. 9, p. 5-22, 2007.

Myers, G. J.; Sandler, C. The art of software testing. John Wiley \& Sons, 2004.

POSIX IEEE Standard 1003.1c-1995. 2003.

Disponível em http://standards.ieee.org/findstds/interps/1003-1c-95_int/ index.html (Acessado em 01-09-2012)

Pressman, R. S. Software engineering: A practitioner's approach. 5 ed. McGraw-Hill Higher Education, 2001.

Quinn, M. J. Parallel programming in C with MPI and OpenMP. McGraw-Hill Education Group, 2003.

Rapps, S.; Weyuker, E. J. Selecting Software Test Data Using Data Flow Information. IEEE Trans. Softw. Eng., v. 11, n. 4, p. 367-375, 1985.

Rungta, N.; Mercer, E. G. Clash of the titans: tools and techniques for hunting bugs in concurrent programs. In: Proc. of PADTAD '09, New York, NY, USA: ACM, 2009, p. 9:1-9:10.

Saini, G. An empirical evaluation of adequacy criteria for testing concurrent programs. Dissertação de Mestrado, Faculty of Graduate School of The University of Texas at Arlington, Arligton, Texas, 2005.

Sarmanho, F. S. Teste de programas concorrentes com memória compartilhada. Dissertação de Mestrado, Instituto de Ciências Matemáticas e de Computação da Universidade de São Paulo, ICMC/USP, São Carlos, SP, 2009.

Sarmanho, F. S.; de Souza, P. S. L.; do Rocio Senger de Souza, S.; da Silva Simão, A. Structural testing for semaphore-based multithread programs. In: ICCS (1), 2008, p. 337-346. 
Seo, H.-S.; Chung, I. S.; Kwon, Y. R. Generating test sequences from statecharts for concurrent program testing. IEICE - Trans. Inf. Syst., v. E89-D, p. 1459-1469, 2006.

Souza, P. L.; Sawabe, E. T.; Simão, A. S.; Vergilio, S. R.; Souza, S. R. S. ValiPVM a graphical tool for structural testing of PVM programs. In: Proceedings of the 15th European PVM/MPI Users' Group Meeting on Recent Advances in Parallel Virtual Machine and Message Passing Interface, Berlin, Heidelberg: Springer-Verlag, 2008a, p. 257-264.

Souza, S. R. S.; , S. R.; Souza, P. S. L.; Simão, A. S.; Hausen, A. C. Structural testing criteria for message-passing parallel programs. Concurr. Comput. : Pract. Exper., v. 20, p. 1893-1916, 2008b.

Souza, S. R. S. Avaliação do custo e eficácia do critério análise de mutantes na atividade de teste de software. Dissertação de Mestrado, Instituto de Ciências Matemáticas e de Computação da Universidade de São Paulo, ICMC/USP, São Carlos, SP, 1996.

Souza, S. R. S.; Fabbri, S. C. P. F.; Barbosa, E. F.; Vincenzi, A. M. R.; Delamaro, M. E.; Jino, M.; Maldonado, J. C. Introdução ao testes de software. v.1, cap. Estudos Teóricos e Experimentais M. E. Delamaro and J.C. Maldonado and M. Jino, p. 251-268, 2007a.

Souza, S. R. S.; Vergilio, S. R.; Souza, P. S. L. Teste de programas concorrentes. In: José Carlos Maldonado; Mario Jino; Márcio Eduardo Delamaro. (Org.). Introdução ao Teste de Software. São Paulo, SP: Elsevier Editora Ltda, p. 231-248, 2007b.

Souza, S. R. S.; Vergilio, S. R.; Souza, P. S. L.; Simão, A. S.; Gonçalves, T. B.; Lima, A. M.; Hausen, A. C. Valipar: A testing tool for message-passing parallel programs. In: 17th International Conference on Software Engineering and Knowledge Engineering (SEKE'2005), 2005, p. 386-391.

Sugeta, T. Proteum-RS/ST : Uma ferramenta para apoiar a validação de especificações statecharts baseada na análise de mutantes. Dissertação de Mestrado, ICMC-USP, São Carlos, SP, Brasil, 1999.

Tanenbaum, A. S. Distributed operating systems. Upper Saddle River, NJ, USA: Prentice-Hall, Inc., 1995.

Taylor, R.; Levine, D.; Kelly, C. Structural testing of concurrent programs. IEEE Transactions on Software Engineering, v. 18, p. 206-215, 1992.

Teixeira, B. C.; Lourenço, J. a.; Farchi, E.; Dias, R. J.; Sousa, D. Detection of transactional memory anomalies using static analysis. In: PDATAD, ACM, 2010, p. 26-36. 
Ural, H.; Yang, B. A structural test selection criterion. Elsevier North-Holland, Inc., v. 28, p. $157-163,1988$.

Valgrind-Developers Valgrind-3.6.1. 2012.

Disponível em http://valgrind.org/

Weyuker, E. J.; Weiss, S. N.; Hamlet, R. G. Comparison of program testing strategies. In: Symposium on Testing, Analysis, and Verification'91, 1991, p. 1-10.

Wilcoxon, F. Individual comparisons by ranking methods. Biometrics, v. 1, p. 80-83, 1945.

Wohlin, C.; Runeson, P.; Höst, M.; Ohlsson, M. C.; Regnell, B.; Wesslén, A. Experimentation in software engineering: an introduction. Norwell, MA, USA: Kluwer Academic Publishers, 2000.

Wong, W. E.; Lei, Y.; Ma, X. Effective generation of test sequences for structural testing of concurrent programs. In: ICECCS, 2005, p. 539-548.

Wong, W. E.; Mathur, A. P.; Maldonado, J. C. Mutation versus all-uses: an empirical evaluation of cost, strength and effectiveness. In: Software Quality and Productivity: Theory, practice and training, London, UK, UK: Chapman \& Hall, 1995, p. 258-265.

Yang, C. D.; Souter, A. L.; Pollock, L. L. All-du-path coverage for parallel programs. In: Proceedings of the 1998 ACM SIGSOFT international symposium on Software testing and analysis, ISSTA '98, New York, NY, USA: ACM, 1998, p. 153-162.

Yang, C. S. D.; Pollock, L. L. All-uses testing of shared memory parallel programs. Software Testing, Verification, And Reliability Journal, v. 13, p. 3-24, 2003.

Yang, Y. Efficient dynamic verification of concurrent programs. Tese de Doutoramento, Salt Lake City, UT, USA, 2009.

Yang, Y. Inspect: A framework for dynamic verification of multithreaded C programs. 2011.

Disponível em http://www.cs.utah.edu/ ${ }^{\sim}$ yuyang/inspect/

Zhu, H. A formal analysis of the subsume relation between software test adequacy criteria. IEEE Transactions on Software Engineering, v. 22, p. 248-255, 1996. 


\section{A.1 Análise Descritiva para o Fator Strength}

A tabela A.1 e o boxplot ilustrado na Figura A.1 correspondem aos dados coletados para o fator strength relacionado à aplicação do conjunto adequado ao critério all-c-uses (ACU) aos demais critérios de teste. Observando o gráfico boxplot, a dificuldade de satisfação dos critérios para esses conjuntos de teste em ordem crescente são all-comm-p-uses (ACPU), all-comm-c-uses (ACCU), all-edges (AE), all-s-edges (AES) e all-s-uses (ASU) ao considerar valor médio por critério, os critérios all-p-uses (APU), all-p-nodes (ANP) e all-nodes (AN) parecem apresentar baixo strength mas pelo fato de apresentarem alguns outliers para a amostra esse resultado deve ser verificado.

Ao aplicar o conjunto de casos de teste adequado ao critério all-comm-c-uses (ACCU) aos demais critérios de teste podemos notar que os critérios all-w-nodes (ANW), all-nodes $(\mathrm{AN})$, all-p-nodes (ANP) e all-comm-p-uses (ACPU) apresentam uma cobertura muito alta com pequena variabilidade nos dados, porém alguns outliers são identificados e devem ser eliminados para que não interfiram nos resultados dos testes estatísticos. Os critérios all-edges (AE), all-s-edges (AES), all-p-uses (APU) e all-sync-uses (ASU) apresentaram alto strength em relação ao critério all-comm-c-uses o que pode indicar que dificilmente estes critérios apresentem alguma relação de inclusão. Esses resultados podem ser observados na Tabela A.2 e no boxplot da Figura A.2. 


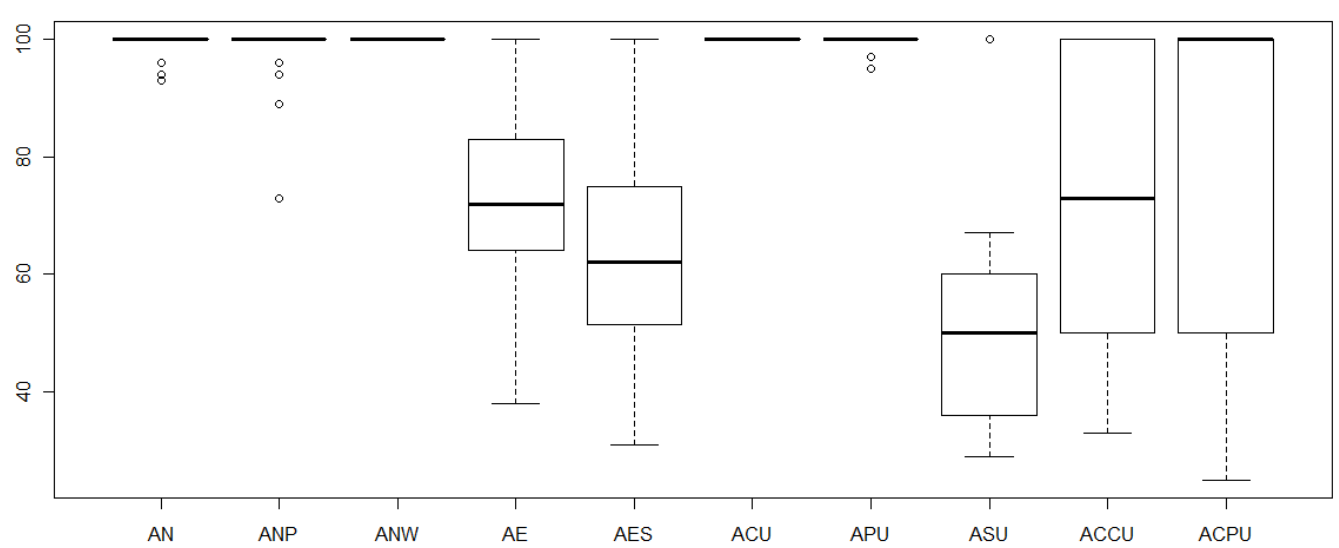

Figura A.1: Dificuldade de satisfação para o critério all-c-uses quando aplicado aos demais critérios.

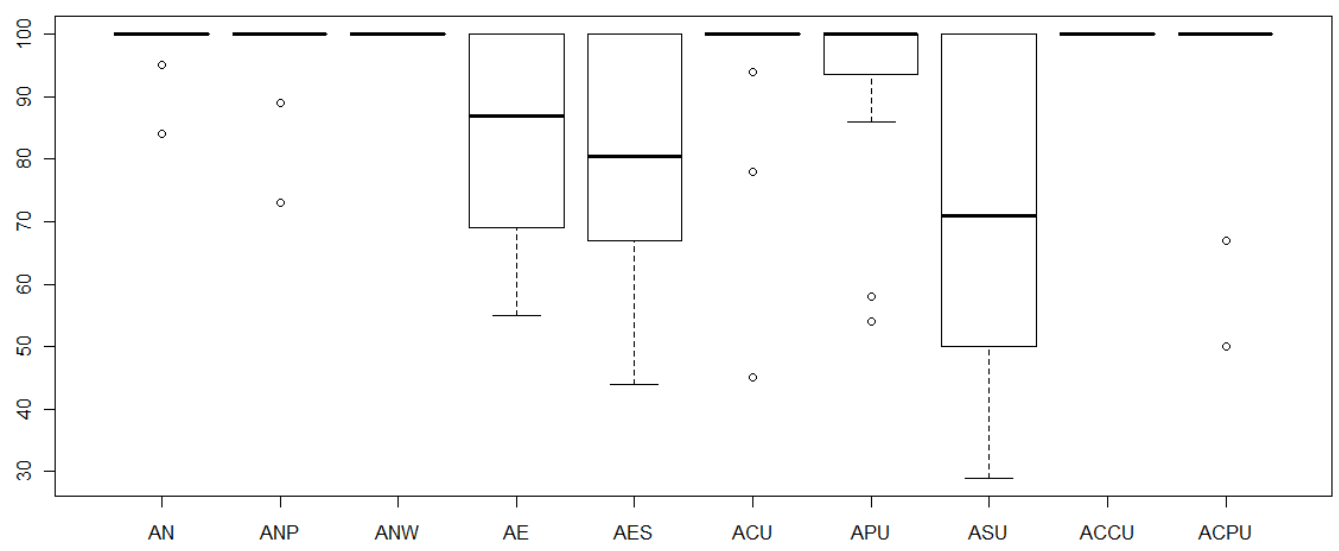

Figura A.2: Dificuldade de satisfação para o critério all-comm-c-uses quando aplicado aos demais critérios.

A Tabela A.3 apresenta os resultados para o fator strength para o conjunto adequado ao critério all-p-uses (APU) e a Figura A.3 mostra o boxplot para esses resultados. Para os critérios all-w-nodes (ANW) e all-c-uses (ACU) o strength obtido foi muito baixo o que sugere a existência de uma relação de inclusão entre esses critérios e o critério all-p-uses, os critérios all-nodes (AN) e all-p-nodes (ANP) também apresentaram uma baixa dificuldade de satisfação, porém por apresentarem outliers, que devem ser analisados antes de se obter qualquer conclusão a respeito desses critérios. Os demais critérios apresentaram baixa cobertura quando submetidos ao conjunto adequado ao critério all-p-uses o que representa um baixo strength para esses critérios. 
Tabela A.1: Aplicação do conjunto de casos de teste adequado ao critério all-c-uses (ACU) aos demais critérios..

\begin{tabular}{|c|c|c|c|c|c|c|c|c|c|c|}
\hline Programa & AN & ANP & ANW & $\mathrm{AE}$ & AES & $\mathrm{ACU}$ & APU & $\mathrm{ASU}$ & $\mathrm{ACCU}$ & $\mathrm{ACPU}$ \\
\hline Sleeping barber & * & $*$ & $*$ & * & 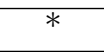 & * & * & $*$ & * & 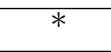 \\
\hline GCD & $100 \%$ & $100 \%$ & $100 \%$ & $72 \%$ & $59 \%$ & $100 \%$ & $100 \%$ & $53 \%$ & $78 \%$ & * \\
\hline Jacobi & $100 \%$ & $*$ & $100 \%$ & $100 \%$ & * & $100 \%$ & * & $*$ & $*$ & * \\
\hline Mmult & * & * & * & * & * & * & * & $*$ & * & * \\
\hline ProdCons & $100 \%$ & $100 \%$ & $100 \%$ & $85 \%$ & $75 \%$ & $100 \%$ & $100 \%$ & $67 \%$ & $100 \%$ & * \\
\hline Carter01 & $100 \%$ & $100 \%$ & $100 \%$ & $100 \%$ & $100 \%$ & $100 \%$ & $100 \%$ & * & * & * \\
\hline Deadlock01 & $*$ & $*$ & $*$ & $*$ & $*$ & * & * & $*$ & * & * \\
\hline Dpor-example1 & $100 \%$ & $100 \%$ & $100 \%$ & $53 \%$ & $43 \%$ & $100 \%$ & * & $33 \%$ & $50 \%$ & * \\
\hline Dpor-example2 & $24 \%$ & $94 \%$ & $92 \%$ & $60 \%$ & $39 \%$ & $100 \%$ & $100 \%$ & * & $73 \%$ & $46 \%$ \\
\hline Dpor-example3 & $100 \%$ & $100 \%$ & $100 \%$ & $69 \%$ & $67 \%$ & $100 \%$ & * & $50 \%$ & $50 \%$ & * \\
\hline Lazy01 & $100 \%$ & $100 \%$ & $100 \%$ & $78 \%$ & $67 \%$ & $100 \%$ & * & $100 \%$ & $100 \%$ & * \\
\hline Phase01 & $100 \%$ & $100 \%$ & $100 \%$ & $69 \%$ & $60 \%$ & $100 \%$ & * & $67 \%$ & $50 \%$ & * \\
\hline Race01 & 7 & * & 7 & * & * & 7 & * & $*$ & * & 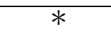 \\
\hline Rafkind01 & $100 \%$ & $100 \%$ & $100 \%$ & $100 \%$ & $100 \%$ & $100 \%$ & * & * & * & * \\
\hline Simple1 & $100 \%$ & $100 \%$ & $100 \%$ & $94 \%$ & $91 \%$ & $100 \%$ & $100 \%$ & $84 \%$ & * & * \\
\hline Stateful01 & $*$ & $*$ & * & $*$ & $*$ & * & $*$ & * & * & * \\
\hline Stateful06 & $100 \%$ & $100 \%$ & $100 \%$ & $68 \%$ & $60 \%$ & $100 \%$ & * & $33 \%$ & $50 \%$ & $33 \%$ \\
\hline Hg01_all_ok & $93 \%$ & $69 \%$ & $100 \%$ & $62 \%$ & $47 \%$ & $100 \%$ & $53 \%$ & $36 \%$ & $67 \%$ & $100 \%$ \\
\hline Hg02_deadlock & $100 \%$ & $100 \%$ & $100 \%$ & $73 \%$ & $67 \%$ & $100 \%$ & * & $56 \%$ & * & * \\
\hline Hg03_inherit & $100 \%$ & $100 \%$ & $100 \%$ & $57 \%$ & $44 \%$ & $100 \%$ & * & $38 \%$ & $100 \%$ & $100 \%$ \\
\hline Hg04_race & $100 \%$ & $100 \%$ & $100 \%$ & $82 \%$ & $75 \%$ & $100 \%$ & * & $50 \%$ & * & $*$ \\
\hline Hg06_readshared & $95 \%$ & $78 \%$ & $100 \%$ & $87 \%$ & $100 \%$ & $100 \%$ & $62 \%$ & $100 \%$ & $100 \%$ & $73 \%$ \\
\hline Tc01_simple_race & $100 \%$ & $100 \%$ & $100 \%$ & $83 \%$ & $77 \%$ & $100 \%$ & $100 \%$ & $67 \%$ & $60 \%$ & $75 \%$ \\
\hline Tc02_simple_tls & $100 \%$ & $100 \%$ & $100 \%$ & $100 \%$ & $100 \%$ & $100 \%$ & * & * & * & * \\
\hline Tc05_simple_race & $100 \%$ & $100 \%$ & $100 \%$ & $100 \%$ & $100 \%$ & $100 \%$ & * & $100 \%$ & $100 \%$ & * \\
\hline Tc06_two_races & $100 \%$ & $100 \%$ & $100 \%$ & $78 \%$ & $67 \%$ & $100 \%$ & * & $100 \%$ & $100 \%$ & * \\
\hline Tc13_laog1 & $100 \%$ & $100 \%$ & $100 \%$ & $37 \%$ & $57 \%$ & $100 \%$ & * & $36 \%$ & * & * \\
\hline Tc16_byterace & $100 \%$ & $100 \%$ & $100 \%$ & $94 \%$ & $91 \%$ & $100 \%$ & $100 \%$ & $84 \%$ & * & * \\
\hline Tc18_semabuse & $100 \%$ & $100 \%$ & $100 \%$ & $100 \%$ & $100 \%$ & $100 \%$ & $100 \%$ & $100 \%$ & * & $100 \%$ \\
\hline Account & $100 \%$ & $100 \%$ & $100 \%$ & $100 \%$ & $100 \%$ & $100 \%$ & $*$ & $*$ & $100 \%$ & $*$ \\
\hline Reorder & $78 \%$ & $64 \%$ & $58 \%$ & $36 \%$ & $05 \%$ & $100 \%$ & $59 \%$ & * & * & * \\
\hline Twostage & $96 \%$ & $96 \%$ & $92 \%$ & $64 \%$ & $49 \%$ & $100 \%$ & $95 \%$ & $48 \%$ & $69 \%$ & $100 \%$ \\
\hline Wronglock & $81 \%$ & $63 \%$ & $54 \%$ & $76 \%$ & $60 \%$ & $100 \%$ & $65 \%$ & $25 \%$ & * & $*$ \\
\hline
\end{tabular}

A Tabela A.4 apresenta os resultados para o fator strength do critério all-sync-uses. Na Figura A.4 é ilustrado o boxplot para o conjunto adequado ao critério all-sync-uses (ASU) quando aplicado aos demais critérios de teste. Por meio da análise do gráfico podemos notar que em geral os critérios apresentam alta cobertura quando submetidos ao conjunto adequado ao critério all-sync-uses, alguns outliers são identificados para os critérios all-edges (AE), all-c-uses (ACU), all-p-uses (APU) e all-comm-p-uses (ACPU) eles devem ser analisados antes de estabelecer relações de inclusão entre os critérios. 
Tabela A.2: Aplicação do conjunto de casos de teste adequado ao critério all-comm-c-uses (ACCU) aos demais critérios.

\begin{tabular}{|c|c|c|c|c|c|c|c|c|c|c|}
\hline Programa & $\mathrm{AN}$ & ANP & ANW & $\mathrm{AE}$ & AES & $\mathrm{ACU}$ & APU & $\mathrm{ASU}$ & $\mathrm{ACCU}$ & $\mathrm{ACPU}$ \\
\hline Sleeping barber & $96 \%$ & $85 \%$ & $100 \%$ & $77 \%$ & $67 \%$ & $100 \%$ & $65 \%$ & $55 \%$ & $100 \%$ & $100 \%$ \\
\hline GCD & * & * & * & * & * & * & * & * & * & * \\
\hline Jacobi & $100 \%$ & $100 \%$ & $100 \%$ & $78 \%$ & $67 \%$ & $100 \%$ & * & $100 \%$ & $100 \%$ & * \\
\hline Mmult & * & * & * & * & * & $*$ & * & * & $*$ & * \\
\hline ProdCons & $100 \%$ & $100 \%$ & $100 \%$ & $100 \%$ & $100 \%$ & $100 \%$ & * & * & $100 \%$ & * \\
\hline Carter01 & $100 \%$ & $100 \%$ & $100 \%$ & $74 \%$ & $67 \%$ & $100 \%$ & * & $44 \%$ & $100 \%$ & $100 \%$ \\
\hline Deadlock01 & $100 \%$ & $100 \%$ & $100 \%$ & $100 \%$ & $100 \%$ & $100 \%$ & * & $100 \%$ & $100 \%$ & * \\
\hline Dpor-example1 & $100 \%$ & $100 \%$ & $100 \%$ & $65 \%$ & $57 \%$ & $100 \%$ & * & $50 \%$ & $100 \%$ & $*$ \\
\hline Dpor-example2 & $100 \%$ & $100 \%$ & $100 \%$ & $85 \%$ & $75 \%$ & $100 \%$ & $100 \%$ & $67 \%$ & $100 \%$ & * \\
\hline Dpor-example3 & $100 \%$ & $100 \%$ & $100 \%$ & $69 \%$ & $67 \%$ & $100 \%$ & * & $50 \%$ & $100 \%$ & * \\
\hline Lazy01 & * & * & * & * & * & * & * & * & * & * \\
\hline Phase01 & $100 \%$ & $100 \%$ & $100 \%$ & $57 \%$ & $44 \%$ & $100 \%$ & * & $38 \%$ & $100 \%$ & $100 \%$ \\
\hline Race01 & $*$ & $*$ & $*$ & $*$ & $*$ & $*$ & * & $*$ & 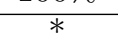 & $*$ \\
\hline Rafkind01 & * & * & * & * & * & * & * & * & * & * \\
\hline Simple1 & $100 \%$ & $100 \%$ & $100 \%$ & $100 \%$ & $100 \%$ & $100 \%$ & * & $100 \%$ & $100 \%$ & * \\
\hline Stateful01 & $100 \%$ & $100 \%$ & $100 \%$ & $100 \%$ & $100 \%$ & $*$ & $100 \%$ & $100 \%$ & $100 \%$ & $*$ \\
\hline Stateful06 & $100 \%$ & $100 \%$ & $100 \%$ & $78 \%$ & $67 \%$ & $100 \%$ & * & $100 \%$ & $100 \%$ & * \\
\hline Hg01_all_ok & * & $*$ & * & * & * & * & * & * & * & * \\
\hline Hg02_deadlock & $*$ & * & * & * & * & * & * & * & * & * \\
\hline Hg03_inherit & $100 \%$ & $100 \%$ & $100 \%$ & $100 \%$ & $100 \%$ & $100 \%$ & $100 \%$ & $100 \%$ & $100 \%$ & $100 \%$ \\
\hline Hg04_race & $100 \%$ & $100 \%$ & $100 \%$ & $100 \%$ & $100 \%$ & * & $100 \%$ & $100 \%$ & $100 \%$ & * \\
\hline Hg06_readshared & * & $*$ & * & * & * & * & $*$ & * & * & * \\
\hline Tc01_simple_race & * & * & * & * & * & * & * & * & * & * \\
\hline Tc02_simple_tls & $*$ & * & * & * & $*$ & * & * & $*$ & * & $*$ \\
\hline Tc05_simple_race & $*$ & * & * & * & * & * & * & * & * & * \\
\hline Tc06_two_races & $95 \%$ & $100 \%$ & $100 \%$ & $89 \%$ & $86 \%$ & $78 \%$ & $86 \%$ & $89 \%$ & $100 \%$ & $100 \%$ \\
\hline Tc13_laog1 & * & * & * & * & * & * & * & * & * & * \\
\hline Tc16_byterace & $100 \%$ & $100 \%$ & $100 \%$ & $100 \%$ & $100 \%$ & * & $100 \%$ & * & $100 \%$ & * \\
\hline Tc18_semabuse & $100 \%$ & $100 \%$ & $100 \%$ & $100 \%$ & $100 \%$ & * & $100 \%$ & * & $100 \%$ & $100 \%$ \\
\hline Account & $*$ & $*$ & $*$ & * & $*$ & * & $*$ & * & $*$ & $*$ \\
\hline Reorder & $95 \%$ & $78 \%$ & $100 \%$ & $87 \%$ & $100 \%$ & $56 \%$ & $52 \%$ & $67 \%$ & $100 \%$ & $73 \%$ \\
\hline Twostage & $100 \%$ & $100 \%$ & $100 \%$ & $56 \%$ & $56 \%$ & $100 \%$ & $100 \%$ & $56 \%$ & $100 \%$ & * \\
\hline Wronglock & $95 \%$ & $100 \%$ & $100 \%$ & $89 \%$ & $86 \%$ & $78 \%$ & $86 \%$ & * & $100 \%$ & $97 \%$ \\
\hline
\end{tabular}

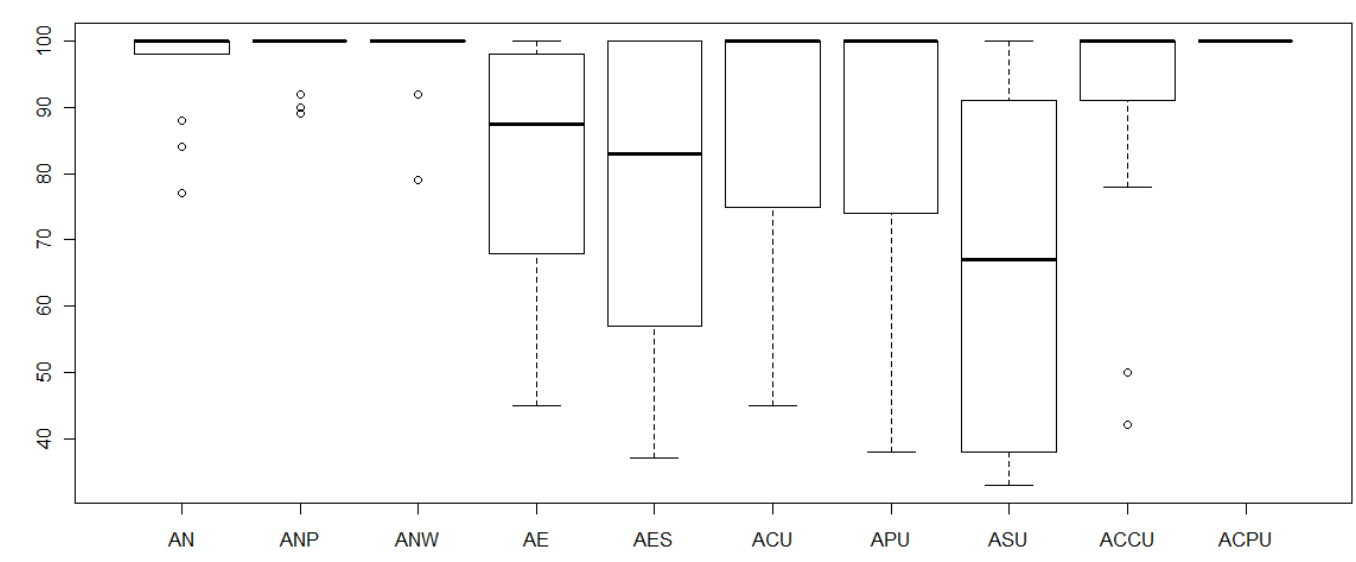

Figura A.5: Dificuldade de satisfação para o critério all-comm-p-uses quando aplicado aos demais critérios. 


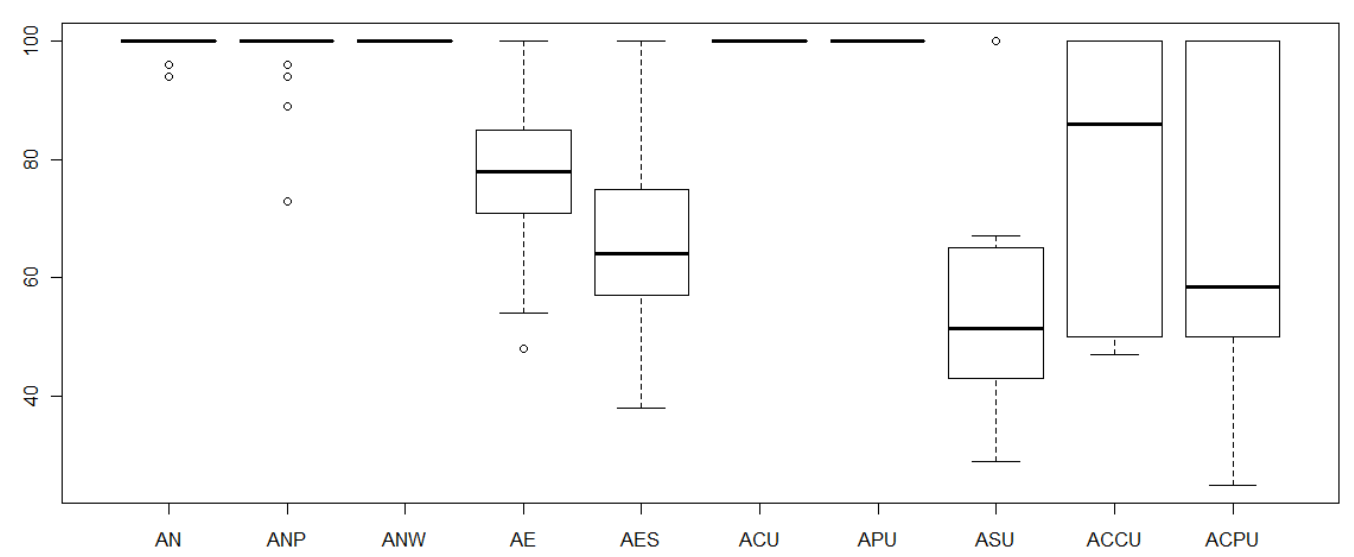

Figura A.3: Dificuldade de satisfação para o critério all-p-uses quando aplicado aos demais critérios.

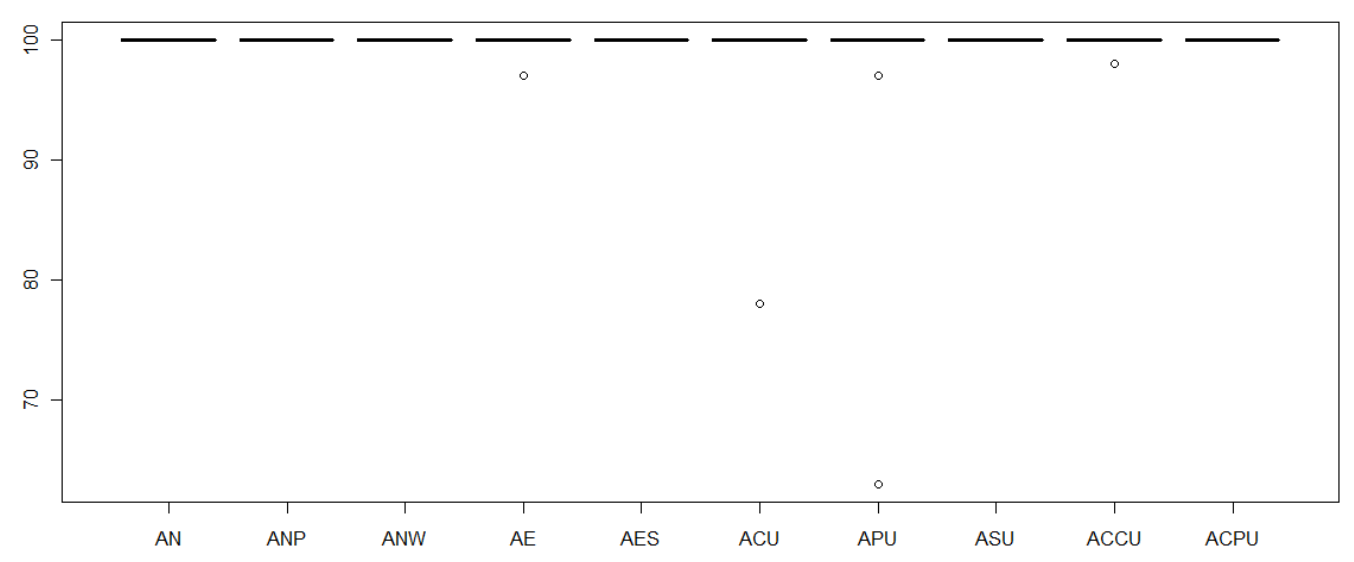

Figura A.4: Dificuldade de satisfação para o critério all-sync-uses quando aplicado aos demais critérios.

Por fim, os resultados correspondentes ao strength para o critério all-comm-p-uses (ACPU) são apresentados na Tabela A.5 e ilustrados no boxplot da Figura A.5. Notam-se coberturas muito altas para os critérios all-nodes (AN) all-p-nodes (ANP) e all-w-nodes (ANW) e pouca variabilidade nos dados da amostra, porém alguns outliers são identificados e devem ser analisados. Os demais critérios apresentam baixa cobertura, sendo o critério all-sync-uses (ASU) o que apresenta menor cobertura e portanto maior strength em seguida os critérios all-s-edges (AES), all-edges (AE), all-p-uses (APU), all-c-uses (ACU) e all-comm-c-uses (ACCU) em ordem crescente de strength. 
Tabela A.3: Aplicação do conjunto de casos de teste adequado ao critério all-p-uses (APU) aos demais critérios.

\begin{tabular}{|c|c|c|c|c|c|c|c|c|c|c|}
\hline Programa & $\overline{\mathrm{AN}}$ & ANP & ANW & $\mathrm{AE}$ & AES & $\overline{\mathrm{ACU}}$ & APU & $\overline{\mathrm{ASU}}$ & $\mathrm{ACCU}$ & $\mathrm{ACPU}$ \\
\hline Sleeping barber & $100 \%$ & $100 \%$ & $100 \%$ & $83 \%$ & $75 \%$ & $*$ & $100 \%$ & $50 \%$ & $83 \%$ & * \\
\hline GCD & * & * & * & * & * & * & * & * & * & * \\
\hline Jacobi & $100 \%$ & $100 \%$ & $100 \%$ & $72 \%$ & $59 \%$ & $100 \%$ & $100 \%$ & $53 \%$ & $78 \%$ & * \\
\hline Mmult & * & * & * & * & * & * & * & * & * & * \\
\hline ProdCons & * & * & * & * & * & * & * & * & * & * \\
\hline Carter01 & $100 \%$ & $100 \%$ & $100 \%$ & $94 \%$ & $91 \%$ & $100 \%$ & $100 \%$ & $84 \%$ & * & * \\
\hline Deadlock01 & * & * & * & * & * & * & * & * & * & * \\
\hline Dpor-example1 & * & * & * & * & * & * & * & * & * & * \\
\hline Dpor-example2 & $100 \%$ & $100 \%$ & $100 \%$ & $100 \%$ & $100 \%$ & * & $100 \%$ & * & $100 \%$ & * \\
\hline Dpor-example3 & $100 \%$ & $100 \%$ & $100 \%$ & $100 \%$ & $100 \%$ & $100 \%$ & $100 \%$ & $100 \%$ & $*$ & $100 \%$ \\
\hline Lazy01 & * & * & * & * & * & * & * & * & * & * \\
\hline Phase01 & $100 \%$ & $100 \%$ & $100 \%$ & $94 \%$ & $91 \%$ & $100 \%$ & $100 \%$ & $84 \%$ & * & * \\
\hline Race01 & $100 \%$ & $100 \%$ & $100 \%$ & $83 \%$ & $77 \%$ & $100 \%$ & $100 \%$ & $67 \%$ & $60 \%$ & $75 \%$ \\
\hline Rafkind01 & * & * & * & * & * & * & * & * & * & * \\
\hline Simple1 & * & * & * & * & * & * & * & * & * & * \\
\hline Stateful01 & * & * & * & * & * & * & * & * & * & * \\
\hline Stateful06 & * & * & * & * & * & * & * & * & * & * \\
\hline Hg01_all_ok & * & * & * & * & * & * & * & * & * & * \\
\hline Hg02_deadlock & * & * & * & * & * & * & * & * & * & * \\
\hline Hg03_inherit & $100 \%$ & $100 \%$ & $100 \%$ & $100 \%$ & $100 \%$ & $100 \%$ & $100 \%$ & $100 \%$ & $100 \%$ & $100 \%$ \\
\hline Hg04_race & * & * & * & * & * & * & * & * & * & * \\
\hline Hg06_readshared & $100 \%$ & $100 \%$ & $100 \%$ & $85 \%$ & $75 \%$ & $100 \%$ & $100 \%$ & $67 \%$ & $100 \%$ & * \\
\hline Tc01_simple_race & $100 \%$ & $100 \%$ & $100 \%$ & $100 \%$ & $100 \%$ & * & $100 \%$ & * & $100 \%$ & $100 \%$ \\
\hline Tc02_simple_tls & $*$ & $*$ & $*$ & $*$ & * & $*$ & $*$ & $*$ & $*$ & $*$ \\
\hline Tc05_simple_race & $100 \%$ & $100 \%$ & $100 \%$ & $100 \%$ & $100 \%$ & $100 \%$ & $100 \%$ & * & * & * \\
\hline Tc06_two_races & $100 \%$ & $100 \%$ & $100 \%$ & $71 \%$ & $18 \%$ & $100 \%$ & $100 \%$ & * & * & * \\
\hline Tc13_laog1 & * & * & * & * & * & * & * & * & * & * \\
\hline Tc16_byterace & $100 \%$ & $100 \%$ & $100 \%$ & $94 \%$ & $91 \%$ & $100 \%$ & $100 \%$ & $82 \%$ & $100 \%$ & $100 \%$ \\
\hline Tc18_semabuse & $100 \%$ & $100 \%$ & $100 \%$ & $78 \%$ & $64 \%$ & $100 \%$ & $100 \%$ & $63 \%$ & $86 \%$ & $100 \%$ \\
\hline Account & $100 \%$ & $100 \%$ & $100 \%$ & $83 \%$ & $75 \%$ & * & $100 \%$ & $50 \%$ & $75 \%$ & * \\
\hline Reorder & $24 \%$ & $94 \%$ & $92 \%$ & $60 \%$ & $39 \%$ & $100 \%$ & $100 \%$ & $*$ & $73 \%$ & $46 \%$ \\
\hline Twostage & * & $*$ & $*$ & * & $*$ & * & * & * & * & $*$ \\
\hline Wronglock & * & * & * & * & * & * & * & * & * & $*$ \\
\hline
\end{tabular}


Tabela A.4: Aplicação do conjunto de casos de teste adequado ao critério all-sync-uses (ASU) aos demais critérios.

\begin{tabular}{|c|c|c|c|c|c|c|c|c|c|c|}
\hline Programa & $\overline{\mathrm{AN}}$ & ANP & ANW & $\mathrm{AE}$ & AES & $\mathrm{ACU}$ & APU & ASU & $\mathrm{ACCU}$ & $\mathrm{ACPU}$ \\
\hline Sleeping barber & $100 \%$ & $100 \%$ & $100 \%$ & $100 \%$ & $100 \%$ & $*$ & $100 \%$ & $100 \%$ & $100 \%$ & $*$ \\
\hline GCD & $*$ & $*$ & $*$ & $*$ & $*$ & $*$ & $*$ & $*$ & $*$ & $*$ \\
\hline Jacobi & $100 \%$ & $100 \%$ & $100 \%$ & $100 \%$ & $100 \%$ & $100 \%$ & $100 \%$ & $100 \%$ & $*$ & $*$ \\
\hline Mmult & $98 \%$ & $85 \%$ & $100 \%$ & $92 \%$ & $93 \%$ & $100 \%$ & $78 \%$ & $100 \%$ & $100 \%$ & $100 \%$ \\
\hline ProdCons & $95 \%$ & $78 \%$ & $100 \%$ & $87 \%$ & $100 \%$ & $100 \%$ & $62 \%$ & $100 \%$ & $100 \%$ & $73 \%$ \\
\hline Carter01 & $*$ & $*$ & $*$ & $*$ & $*$ & $*$ & $*$ & $*$ & $*$ & $*$ \\
\hline Deadlock01 & $100 \%$ & $100 \%$ & $100 \%$ & $100 \%$ & $100 \%$ & $100 \%$ & $100 \%$ & $100 \%$ & $*$ & $100 \%$ \\
\hline Dpor-example1 & $100 \%$ & $100 \%$ & $100 \%$ & $100 \%$ & $100 \%$ & $100 \%$ & $*$ & $100 \%$ & $100 \%$ & $*$ \\
\hline Dpor-example2 & $100 \%$ & $100 \%$ & $100 \%$ & $100 \%$ & $100 \%$ & $100 \%$ & $*$ & $100 \%$ & $100 \%$ & $100 \%$ \\
\hline Dpor-example3 & $100 \%$ & $100 \%$ & $100 \%$ & $100 \%$ & $100 \%$ & $100 \%$ & $100 \%$ & $100 \%$ & $*$ & $*$ \\
\hline Lazy01 & $93 \%$ & $70 \%$ & $100 \%$ & $82 \%$ & $91 \%$ & $100 \%$ & $100 \%$ & $100 \%$ & $*$ & $*$ \\
\hline Phase01 & $100 \%$ & $100 \%$ & $100 \%$ & $100 \%$ & $100 \%$ & $100 \%$ & $100 \%$ & $100 \%$ & $100 \%$ & $100 \%$ \\
\hline Race01 & $100 \%$ & $100 \%$ & $100 \%$ & $100 \%$ & $100 \%$ & $100 \%$ & $100 \%$ & $100 \%$ & $100 \%$ & $*$ \\
\hline Rafkind01 & $100 \%$ & $100 \%$ & $100 \%$ & $100 \%$ & $100 \%$ & $100 \%$ & $100 \%$ & $100 \%$ & $98 \%$ & $*$ \\
\hline Simple1 & $100 \%$ & $100 \%$ & $100 \%$ & $100 \%$ & $100 \%$ & $100 \%$ & $*$ & $100 \%$ & $100 \%$ & $*$ \\
\hline Stateful01 & $*$ & $*$ & $*$ & $*$ & $*$ & $*$ & $*$ & $*$ & $*$ & $*$ \\
\hline Stateful06 & $100 \%$ & $100 \%$ & $100 \%$ & $78 \%$ & $67 \%$ & $100 \%$ & * & $100 \%$ & $100 \%$ & $*$ \\
\hline Hg01_all_ok & $100 \%$ & $100 \%$ & $100 \%$ & $100 \%$ & $100 \%$ & $*$ & $100 \%$ & $100 \%$ & $100 \%$ & $*$ \\
\hline Hg02_deadlock & $100 \%$ & $100 \%$ & $100 \%$ & $100 \%$ & $100 \%$ & $100 \%$ & $*$ & $100 \%$ & $100 \%$ & $100 \%$ \\
\hline Hg03_inherit & $100 \%$ & $100 \%$ & $100 \%$ & $100 \%$ & $100 \%$ & $100 \%$ & * & $100 \%$ & $*$ & $*$ \\
\hline Hg04_race & $*$ & $*$ & $*$ & $*$ & $*$ & $*$ & $*$ & $*$ & $*$ & $*$ \\
\hline Hg06_readshared & $100 \%$ & $100 \%$ & $100 \%$ & $100 \%$ & $100 \%$ & $100 \%$ & $*$ & $100 \%$ & $100 \%$ & $*$ \\
\hline Tc01_simple_race & $95 \%$ & $100 \%$ & $100 \%$ & $96 \%$ & $95 \%$ & $78 \%$ & $63 \%$ & $100 \%$ & $100 \%$ & $100 \%$ \\
\hline Tc02_simple_tls & $*$ & $*$ & $*$ & $*$ & $*$ & $*$ & $*$ & $*$ & $*$ & $*$ \\
\hline Tc05_simple_race & $100 \%$ & $100 \%$ & $100 \%$ & $100 \%$ & $100 \%$ & $100 \%$ & $*$ & $100 \%$ & $100 \%$ & $*$ \\
\hline Tc06_two_races & $100 \%$ & $100 \%$ & $100 \%$ & $100 \%$ & $100 \%$ & $100 \%$ & $*$ & $100 \%$ & $*$ & $*$ \\
\hline Tc13_laog1 & $*$ & $*$ & $*$ & $*$ & $*$ & $*$ & $*$ & $*$ & $*$ & * \\
\hline Tc16_byterace & $*$ & $*$ & $*$ & $*$ & $*$ & $*$ & $*$ & $*$ & $*$ & $*$ \\
\hline Tc18_semabuse & $100 \%$ & $100 \%$ & $100 \%$ & $100 \%$ & $100 \%$ & $*$ & $*$ & $100 \%$ & $*$ & $*$ \\
\hline Account & $100 \%$ & $100 \%$ & $100 \%$ & $100 \%$ & $100 \%$ & $100 \%$ & $*$ & $100 \%$ & $*$ & $*$ \\
\hline Reorder & $*$ & $*$ & $*$ & $*$ & $*$ & $*$ & $*$ & $*$ & $*$ & $*$ \\
\hline Twostage & $100 \%$ & $100 \%$ & $100 \%$ & $100 \%$ & $100 \%$ & $100 \%$ & $*$ & $100 \%$ & $100 \%$ & $*$ \\
\hline Wronglock & $*$ & $*$ & $*$ & $*$ & $*$ & $*$ & $*$ & $*$ & $*$ & $*$ \\
\hline
\end{tabular}


Tabela A.5: Aplicação do conjunto de casos de teste adequado ao critério all-comm-p-uses (ACPU) aos demais critérios.

\begin{tabular}{|c|c|c|c|c|c|c|c|c|c|c|}
\hline Programa & $\overline{\mathrm{AN}}$ & ANP & ANW & $\overline{\mathrm{AE}}$ & AES & $\mathrm{ACU}$ & $\mathrm{APU}$ & ASU & $\mathrm{ACCU}$ & $\overline{\mathrm{ACPU}}$ \\
\hline Sleeping barber & $*$ & $*$ & * & $*$ & $*$ & * & * & $*$ & $*$ & $*$ \\
\hline GCD & * & $*$ & $*$ & $*$ & $*$ & $*$ & $*$ & $*$ & $*$ & * \\
\hline Jacobi & * & * & $*$ & $*$ & * & $*$ & $*$ & * & $*$ & * \\
\hline Mmult & $*$ & $*$ & $*$ & $*$ & $*$ & $*$ & $*$ & $*$ & $*$ & $*$ \\
\hline ProdCons & $98 \%$ & $89 \%$ & $100 \%$ & $93 \%$ & $100 \%$ & $56 \%$ & $62 \%$ & $67 \%$ & $100 \%$ & $91 \%$ \\
\hline Carter01 & $100 \%$ & $100 \%$ & $100 \%$ & $95 \%$ & $93 \%$ & $100 \%$ & $*$ & $89 \%$ & $50 \%$ & $100 \%$ \\
\hline Deadlock01 & $*$ & $*$ & $*$ & $*$ & $*$ & $*$ & $*$ & * & $*$ & * \\
\hline Dpor-example1 & $93 \%$ & $69 \%$ & $100 \%$ & $62 \%$ & $47 \%$ & $100 \%$ & $53 \%$ & $36 \%$ & $67 \%$ & $100 \%$ \\
\hline Dpor-example2 & $99 \%$ & $100 \%$ & $93 \%$ & $88 \%$ & $83 \%$ & $96 \%$ & $91 \%$ & * & $92 \%$ & $100 \%$ \\
\hline Dpor-example3 & $*$ & $*$ & $*$ & $*$ & $*$ & $*$ & $*$ & * & $*$ & * \\
\hline Lazy01 & $100 \%$ & $100 \%$ & $100 \%$ & $100 \%$ & $100 \%$ & $*$ & $100 \%$ & * & $100 \%$ & $100 \%$ \\
\hline Phase01 & $*$ & $*$ & $*$ & * & $*$ & $*$ & $*$ & $*$ & $*$ & $*$ \\
\hline Race01 & $100 \%$ & $100 \%$ & $100 \%$ & $100 \%$ & $100 \%$ & $100 \%$ & $100 \%$ & $100 \%$ & $100 \%$ & $100 \%$ \\
\hline Rafkind01 & $*$ & $*$ & $*$ & $*$ & $*$ & $*$ & $*$ & * & $*$ & $*$ \\
\hline Simple1 & $*$ & * & $*$ & * & $*$ & * & $*$ & $*$ & * & $*$ \\
\hline Stateful01 & $*$ & * & $*$ & $*$ & $*$ & $*$ & $*$ & $*$ & $*$ & $*$ \\
\hline Stateful06 & * & * & * & * & * & * & * & * & * & * \\
\hline Hg01_all_ok & * & * & * & * & $*$ & $*$ & * & $*$ & $*$ & * \\
\hline Hg02_deadlock & * & * & * & * & * & * & $*$ & $*$ & $*$ & * \\
\hline Hg03_inherit & * & $*$ & $*$ & $*$ & * & * & $*$ & * & $*$ & * \\
\hline Hg04_race & * & * & $*$ & $*$ & $*$ & $*$ & $*$ & $*$ & $*$ & * \\
\hline Hg06_readshared & * & * & $*$ & * & * & * & * & * & * & * \\
\hline Tc01_simple_race & $*$ & * & * & * & $*$ & $*$ & $*$ & $*$ & $*$ & $*$ \\
\hline Tc02_simple_tls & $*$ & * & $*$ & $*$ & $*$ & $*$ & $*$ & $*$ & $*$ & $*$ \\
\hline Tc05_simple_race & $*$ & * & $*$ & $*$ & $*$ & * & * & $*$ & $*$ & $*$ \\
\hline Tc06_two_races & $77 \%$ & $92 \%$ & $79 \%$ & $45 \%$ & $37 \%$ & $55 \%$ & $38 \%$ & $33 \%$ & $42 \%$ & $100 \%$ \\
\hline Tc13_laog1 & $100 \%$ & $100 \%$ & $100 \%$ & $57 \%$ & $44 \%$ & $100 \%$ & $*$ & $38 \%$ & $100 \%$ & $100 \%$ \\
\hline Tc16_byterace & $*$ & * & * & * & * & * & * & * & * & * \\
\hline Tc18_semabuse & * & $*$ & $*$ & $*$ & $*$ & * & * & * & $*$ & $*$ \\
\hline Account & $*$ & * & $*$ & $*$ & * & * & $*$ & * & $*$ & $*$ \\
\hline Reorder & $*$ & * & $*$ & $*$ & * & $*$ & * & $*$ & * & $*$ \\
\hline Twostage & $100 \%$ & $100 \%$ & $100 \%$ & $100 \%$ & $100 \%$ & $100 \%$ & $100 \%$ & $100 \%$ & $100 \%$ & $100 \%$ \\
\hline Wronglock & $*$ & $*$ & $*$ & $*$ & $*$ & $*$ & $*$ & $*$ & $*$ & $*$ \\
\hline
\end{tabular}

